\title{
Progress on Gust Load Alleviation Wind Tunnel Experiment and Aeroservoelastic Model Validation for a Flexible Wing with Variable Camber Continuous Trailing Edge Flap System
}

\author{
Nhan Nguyen* \\ Nicholas Cramer ${ }^{\dagger}$ \\ Kelley Hashemi ${ }^{\ddagger}$ \\ NASA Ames Research Center, Moffett Field, CA 94035 \\ Michael Drew ${ }^{\S}$ \\ Juntao Xiong \\ Stinger Ghaffarian Technologies, Inc., Moffett Field, CA 94035 \\ Tyler Mundt ${ }^{\|}$ \\ Marat Mor** \\ Eli Livne ${ }^{\dagger \dagger}$ \\ University of Washington, Seattle, WA 98195 \\ Joseph Jackson ${ }^{\ddagger \ddagger}$ \\ Richard Wise \\ Adam Shultz \\ Jovan Boskovic \\ Scientific Systems Company, Inc., Woburn, MA 01801
}

\begin{abstract}
This paper discusses a wind tunnel experiment of active gust load alleviation of a flexible wing which took place at University of Washington (UW) in 2019. The experiment performed under a NASA SBIR contract with Scientific Systems Company, Inc (SSCI). The objective of the experiment is to demonstrate active controls of the Variable Camber Continuous Trailing Edge Flap (VCCTEF) system for gust load alleviation and realtime drag optimization. The wind tunnel model is a 8.2\% sub-scale Common Research Model (CRM) wing. The wing structure is designed to provide a substantial degree of flexibility to represent that of a modern highaspect ratio wing. Eight active control surfaces are employed in the VCCTEF. A new gust generator system was designed and installed by UW under a sub-contract with SSCI. The first test entry started in July 2019 and ended in September 2019. During this test entry, many significant issues were found with the hardware and software. The significant issues with the servos prevented the test objective from being completed. A follow-up second test entry in 2020 is being planned. The wing system is being repaired by SSCI. This paper reports on the progress of this experimental effort and the aeroservoelastic (ASE) model validation which was conducted during the test entry.
\end{abstract}

\footnotetext{
*Senior Research Scientist and Technical Group Lead, Intelligent Systems Division, nhan.t.nguyen@nasa.gov

†Aerospace Engineer, Intelligent Systems Division, nicholas.b.cramer@nasa.gov

$\ddagger$ Aerospace Engineer, Intelligent Systems Division, kelley.e.hashemi@nasa.gov

§Aerospace Engineer, Intelligent Systems Division, michael.c.drew@nasa.gov

๑Aerospace Engineer, Intelligent Systems Division, juntao.xiong@nasa.gov

" Graduate student, William E. Boeing Department of Aeronautics and Astronautics, tmundt94@uw.edu

**Affiliate Associate Professor, William E. Boeing Department of Aeronautics and Astronautics, mmor@uw.edu

${ }^{\dagger \dagger}$ Boeing Endowed Professor, William E. Boeing Department of Aeronautics and Astronautics, eli@aa.washington.edu

$\ddagger \ddagger$ Lead Research Engineer, Scientific Systems Company, Inc., joseph.jackson@ssci.com

Lead Integration Engineer, Scientific Systems Company, Inc., rwise@ ssci.com

Senior Research Engineer, Scientific Systems Company, Inc., Abraham.Shultz@ssci.com

Principal Research Engineer and Senior Group Leader, Scientific Systems Company, Inc., jovan@ ssci.com
} 


\section{Introduction}

Air vehicles are typically designed to maintain sufficient structural rigidity for safe load-carrying capacity. Advanced composite materials have gained widespread adoption as materials of choice for modern airframe structures. This adoption is driven by the need to reduce airframe operational empty weight $(\mathrm{OEW})$ which is a major consideration for improving energy efficiency. Composite structures tend to exhibit less structural rigidity while providing the same load-carrying capacity. An example of light-weight airframe design is the Boeing 787 Dreamliner aircraft, which has a more flexible wing structure than older-generation aircraft. This increased structural flexibility afforded by modern materials could be exploited to improve aerodynamic efficiency of future air vehicle concepts. ${ }^{1}$

As the wing flexibility increases, aeroelastic interactions with aerodynamic forces and moments can alter aircraft aerodynamics significantly, thereby degrading aerodynamic efficiency. Increased drag, hence increased fuel burn, is one such potential consequence. Without means for aeroelastic compensation, the benefit of weight reduction afforded by composite materials could be offset by suboptimal aerodynamic performance at off-design flight conditions. Performance Adaptive Aeroelastic Wing (PAAW) technology can potentially address these technical challenges for future flexible wing transports. PAAW technology leverages multi-disciplinary solutions to maximize the aerodynamic performance payoff of future adaptive wing design, while simultaneously addressing operational constraints that can prevent optimal aerodynamic performance from being realized.

To address the performance aspects of wing flexibility in transport design, NASA developed a wing shaping control concept called the variable camber continuous trailing edge flap (VCCTEF) in 2010. ${ }^{1-3}$ This study shows that highly flexible wing aerodynamic surfaces can be elastically shaped in-flight by active controls of wing twist and bending to optimize the wing shape for improved aerodynamic efficiency. Subsequently, this study has been further investigated since 2011. Boeing Research and Technology collaborated with NASA under a two-phase study to further develop the VCCTEF concept. The Phase I study was performed during 2012 to refine the initial VCCTEF concept and develop actuation mechanisms. ${ }^{4,5}$ The Phase II study was a two-year effort from 2013 to 2014. The objectives of the Phase II study were to conduct aeroelastic analysis and flutter suppression control as well as two wind tunnel experiments to validate the VCCTEF design for cruise and high-lift performance. ${ }^{6,8,21}$

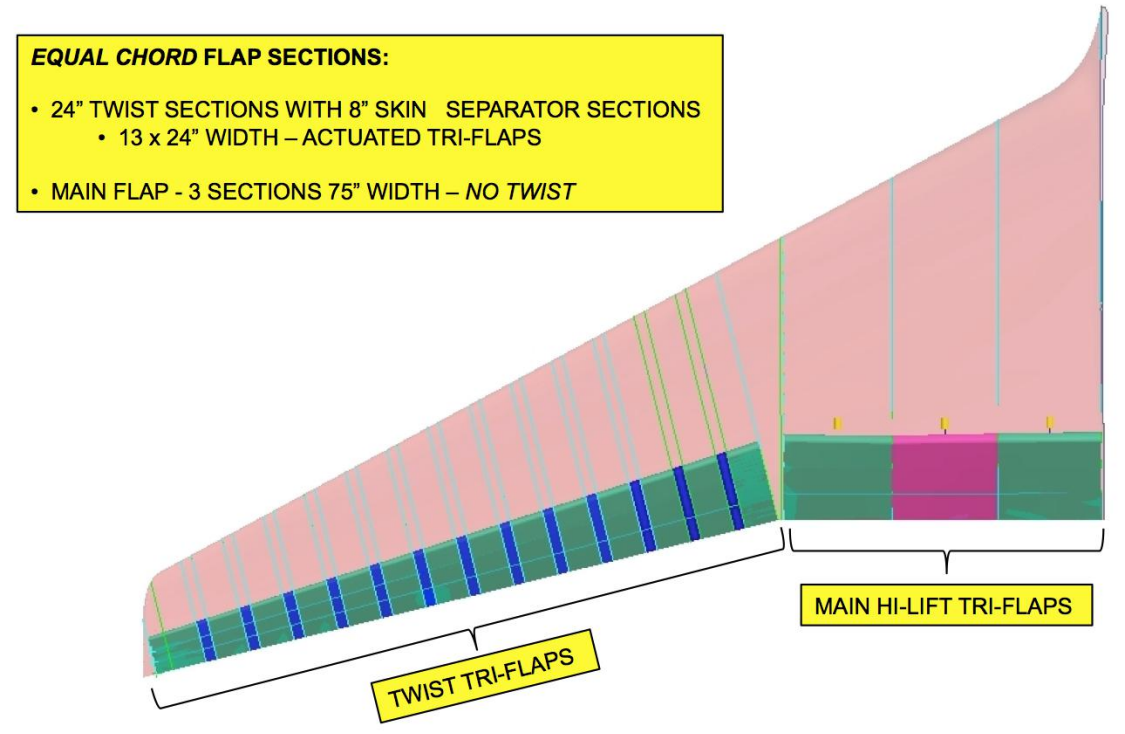

Figure 1. Typical Transport Wing Configured with the Variable Camber Continuous Trailing Edge Flap

The VCCTEF system, as shown in Fig. 1, employs a light-weight shaped memory alloy (SMA) technology for actuation and three individual chordwise segments, as shown in Fig. 2, to provide a variable camber aerodynamic surface to shape the chordwise pressure distribution for improved aerodynamic performance. The VCCTEF system is divided into multiple sections attached to the outer wing and the inner wing. Each spanwise flap section has three cambered flap segments that can be individually commanded. These cambered flaps are joined to the next section by a flexible transition material installed with the same shape as the camber and thus providing a continuous trailing edge flap throughout the wing span with no drag-producing gaps. ${ }^{5}$ This continuous trailing edge flap design combined with the flap camber result in lower drag increase during flap deflections. In addition, it also offers a potential noise 
reduction benefit. This results in the ability to control the wing twist shape as a function of span, resulting in a change to the wing twist to establish the best lift-to-drag ratio $(L / D)$ at any aircraft gross weight or mission segment. Current wing twist on commercial transports is permanently set for one cruise configuration, usually for a $50 \%$ loading or midpoint on the gross weight schedule. The VCCTEF offers a mission-adaptive wing capability by enabling a different wing twist setting for each gross weight condition and also different settings for climb, cruise and descent, a major factor in obtaining best $L / D$ conditions.

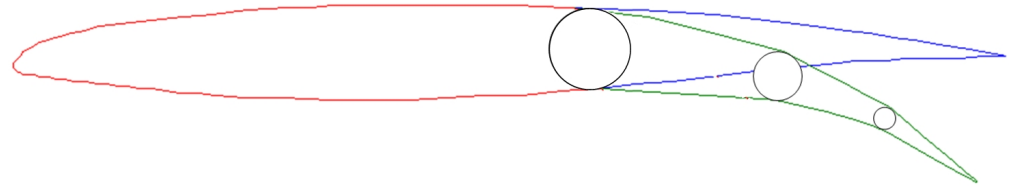

Figure 2. Three-Segment Variable Camber Flap

Adaptive wing technologies such as the VCCTEF are envisioned to provide the ability to automatically reconfigure a wing configuration in-flight to maximize the cruise aerodynamic efficiency. In an ideal setting, an integrated adaptive wing design would incorporate many subsystems including actuation mechanisms, sensors, flight control system, flight management system, and software algorithms. The full potential of adaptive wing technologies can be further realized if these subsystems can be designed synergistically to achieve real-time drag optimization in-flight. ${ }^{9,10}$

Moreover, as the wing aspect ratio increases, structural flexibility becomes a more important consideration. Two issues are at hands. The changes in the wing shape at off-design cruise conditions cause the wing aerodynamics to be less efficient than the aerodynamics of a stiffer wing. This necessitates a real-time drag optimization technology in modern transports. The second issue deals with the ride quality of aircraft equipped with flexible wings. As structural flexibility increases, susceptibility to gust encounters also increases. The atmospheric turbulence provides a source of excitation to the wing structure. Structural vibration of the flexible wing can cause increased passenger ride discomfort and adversely affect the ride quality. To reduce passenger ride discomfort, active gust load alleviation technology becomes an important requirement for flight control systems in modern transports.

In order to mature the VCCTEF technology, experimental efforts have been conducted since 2013 through four wind tunnel tests at UW. To date, three of these wind tunnel tests have been completed. The first wind tunnel test was conducted in 2013 to assess the aerodynamic performance of the VCCTEF. ${ }^{11,22}$ This test shows that up to 6\% drag reduction has been observed in the experimental data for the tested VCCTEF configurations. The second wind tunnel test was conducted in 2014 to assess the high-lift performance of the VCCTEF. ${ }^{13,14}$ The high-lift wind tunnel model incorporates one variable camber Fowler flap at the inboard as part of the VCCTEF system along with a variable camber Krueger continuous leading edge slat. The test results show that the VCCTEF can achieve the necessary high-lift $C_{L_{\max }}$ requirement. The third wind tunnel test campaign was conducted in 2017 and 2018 to validate the real-time drag optimization strategy. This test successfully demonstrates the real-time drag optimization which was able to achieve up to $9.4 \%$ drag reduction for off-design cruise conditions. ${ }^{15,16}$ The fourth wind tunnel test is the subject of this paper which is to validate a multi-objective gust load alleviation/drag optimization control technology. The primary objective of the fourth wind tunnel test is gust load alleviation with drag optimization as a secondary objective. The multi-objective control technology would enable both of these objectives to be achieved simultaneously in real time. ${ }^{17-20}$

This paper reports the wind tunnel test for gust load alleviation which took place in 2019 but was not completed. In particular, it discusses the progress being made in 2019 toward the completion of the test objective, and some aspects of validation of the ASE model developed by NASA in support of the active control design efforts conducted by both SSCI and NASA.

\section{Wind Tunnel Model Description}

The wind tunnel model is a sub-scale model of a Common Research Model (CRM) wing. ${ }^{20}$ Comparing to the wing planform of the full-scale CRM wing starting at the side-of-body wing station, ${ }^{20}$ the wind tunnel model is a $8.2 \%$ subscale CRM wing. This CRM wing is designed to have about a wing tip deflection of $10 \%$ of the wing semi-span to represent a typical wing flexibility of the current state-of-the-art high aspect ratio wings of modern transport aircraft such as the Boeing 787. The wing is 85 inches in length with a leading edge sweep angle of $35^{\circ}$ at the quarter chord. The wing physical parameters are provided in Table 1.

The original CRM wing geometry ${ }^{20}$ is a cruise shape outer mold line (OML) with a jig-shape twist to account 
for the wing aeroelastic deflection at the design cruise condition. Modifications are made to the original CRM wing geometry to account for the increase in the wing flexibility with a $10 \%$ wing tip deflection. The cruise shape OML is removed by flattening the original CRM wing geometry. A new jig-shape twist is then applied to the flattened OML. The new jig-shape twist is computed by an aero-structural optimization using the CART3D Euler CFD code coupled to a notional structural beam model of the wing for the design $C_{L}=0.5$ at Mach 0.3 . The optimized incremental jig-shape twist is shown in Fig. 3. This incremental jig-shape twist is then applied to the original design jig-shape twist. $^{20}$

\begin{tabular}{|l|l|}
\hline Wing Semi-Span & 85 inches \\
\hline Location of Yehudi Break & 25.083 inches \\
\hline Root Chord & 38.101 inches \\
\hline Tip Chord & 8.806 inches \\
\hline Chord at Yehudi Break & 23.425 inch \\
\hline Leading Edge Sweep & $37.1818^{\circ}$ \\
\hline Wing Area & $12.0608 \mathrm{ft}^{2}$ \\
\hline Mean Aerodynamic Chord & 23.5221 inches \\
\hline Aspect Ratio & 8.3201 \\
\hline
\end{tabular}

Table 1. CRM Wing Physical Parameters

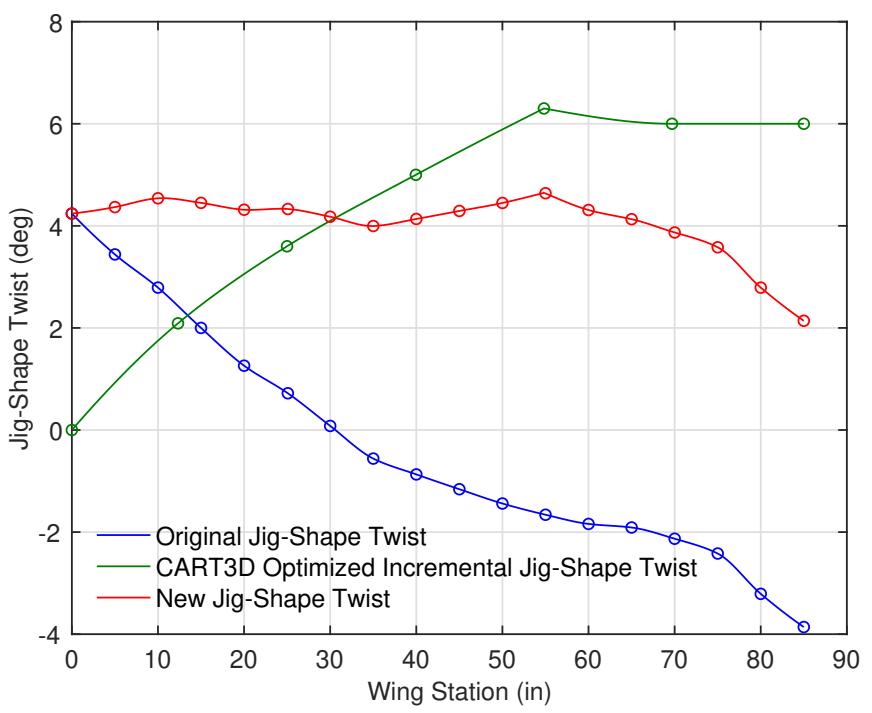

Figure 3. CRM Wing Optimized Jig-Shape Twist

\section{A. CRM Wing Design for 2018 Wind Tunnel Test}

The basis for the CRM wing design for the gust load alleviation wind tunnel test can be traced back to the original CRM wing design for the real-time drag optimization wind tunnel test in 2018. In the 2018 wind tunnel test, the wing is constructed using a foam core covered with a two-layer fiberglass skin as shown in Fig. 4. The continuous fiberglass skin construction provides a clean aerodynamic surface for accurate lift and drag measurements and does not follow a common beam / aerodynamic shell design practice for subsonic aeroelastic wind tunnel models. The VCCTEF system comprises six active two-segment control surfaces. These 12 control surfaces are driven by 12 independent servoactuators for the real-time drag optimization strategy. The flap segments and actuator housing are made of aluminum. The flap gaps are sealed with elastomer transition inserts to provide the continuous trailing edge. The 12 flap segments are numbered from 1 to 6 from inboard to outboard. The inner cambered segment is designated with the letter A and the outer cambered segment is designated with the letter B. The flap numbering is shown in Fig. $5 .{ }^{15}$ 


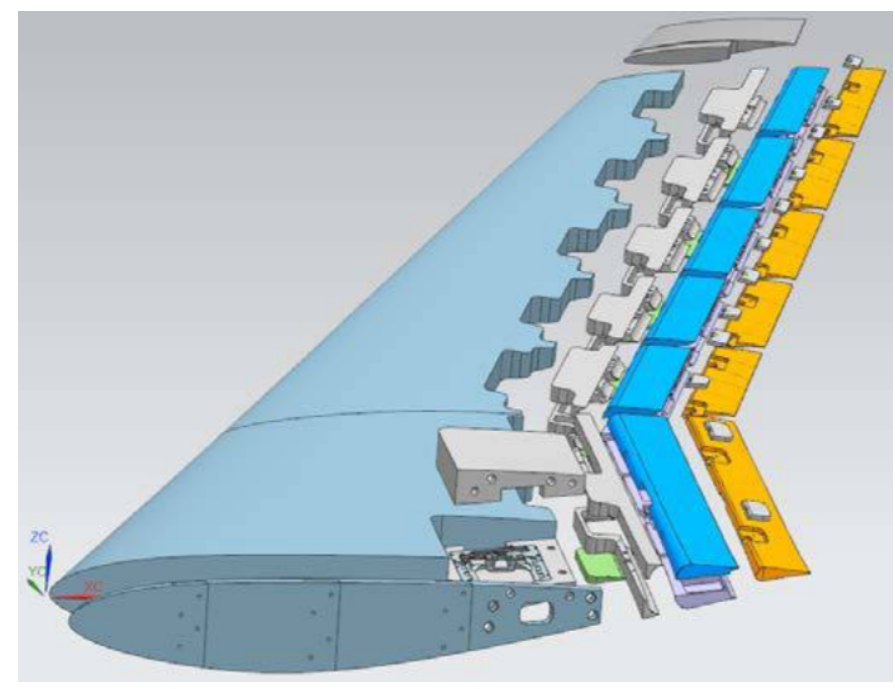

Figure 4. Exploded View of CRM Wing with VCCTEF System

The wing is attached to the sidewall balance in the test section in a horizontal position via a steel tube to which the wing is bolted at the load block, as shown in Fig. $5 .{ }^{15}$ The sidewall balance provides the lift, drag, and pitching moment measurements. A control system computer is interfaced with the wind tunnel data acquisition system to drive the angle of attack and flap positions during the experiment.

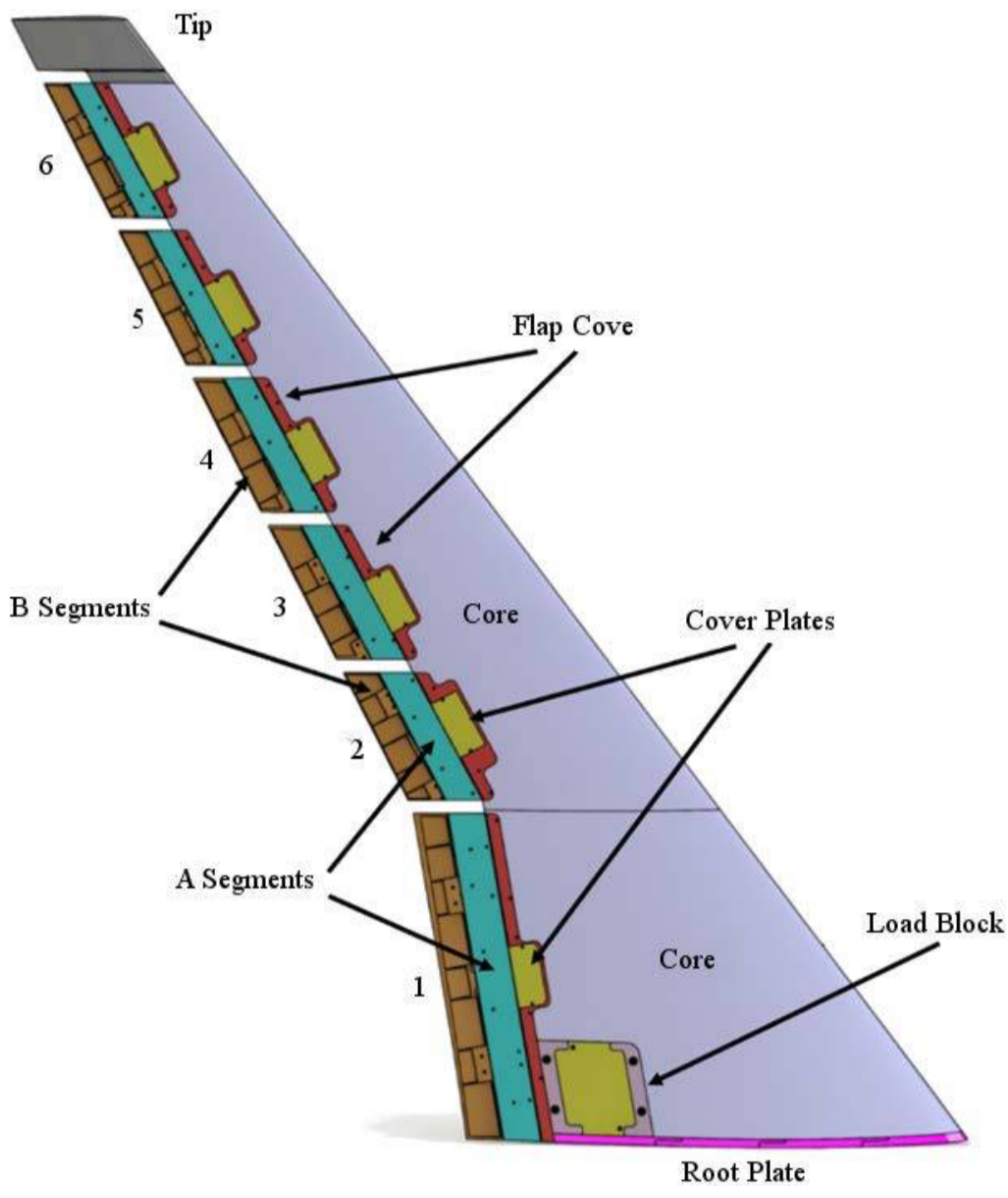

Figure 5. CRM Wing Planform 
The sidewall balance measures 6 force and moment components. The wind tunnel test only concerns with the lift and drag measurements. To alleviate the boundary layer effect in the test section, the wing is mounted 6 inches off the sidewall through a hole in a non-metric splitter plate, as shown in Fig. $6 .{ }^{15}$

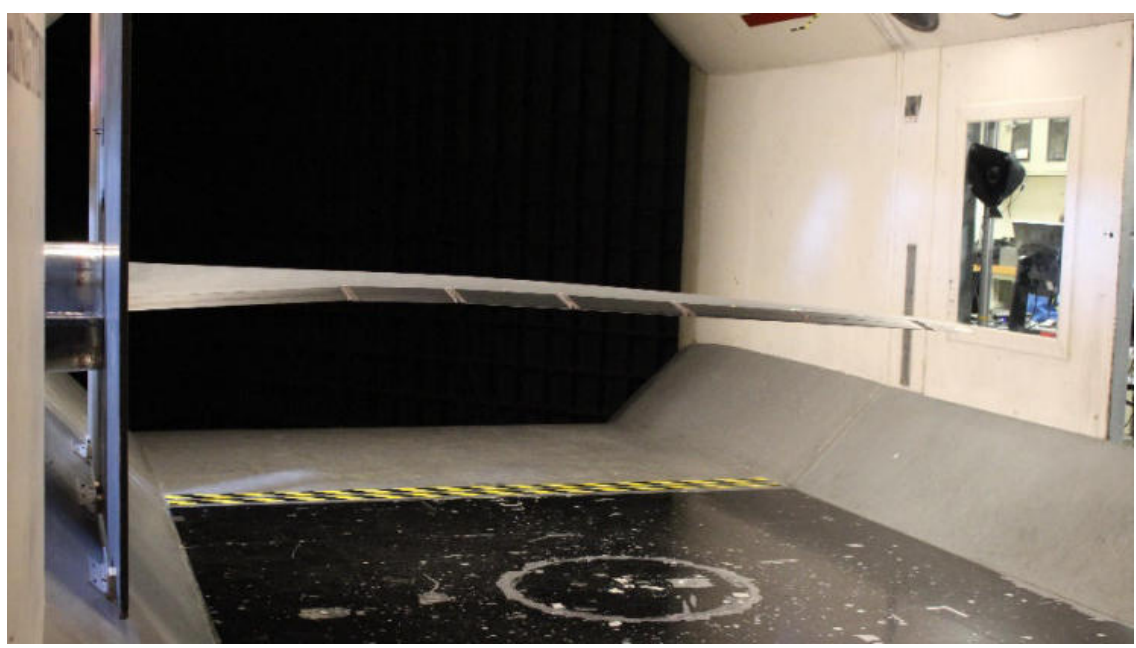

Figure 6. CRM Wing Installed in Test Section (September 2017)

The drag optimization objective of the 2018 wind tunnel test requires a clean aerodynamic surface. Therefore, the flap actuators are designed to be packaged inside the outer mold line of the wind tunnel model with no external protuberances. The requirement for the flap actuators to be able to fit in the small internal flap volumes presents a significant challenge in the design which places limitations on the size and power of the actuators. A typical flap actuator assembly is shown in Figs. 7 and 8. Each actuator is installed in an aluminum cradle, along with an optical encoder sensor to measure the actuator extension.

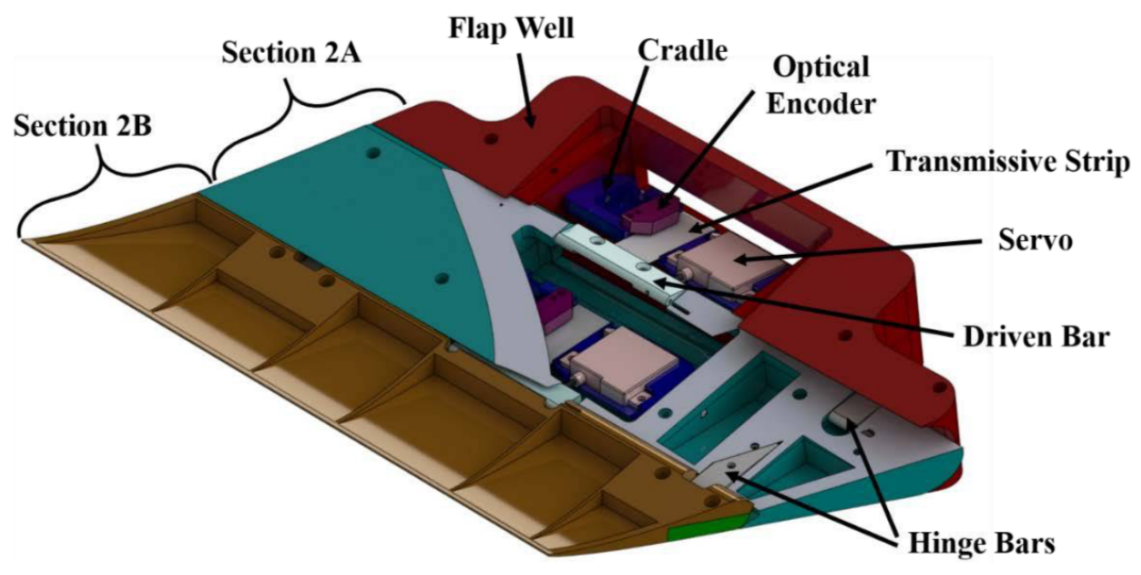

Figure 7. Exploded View of VCCTEF Actuator Assembly of Flaps 2A and 2B

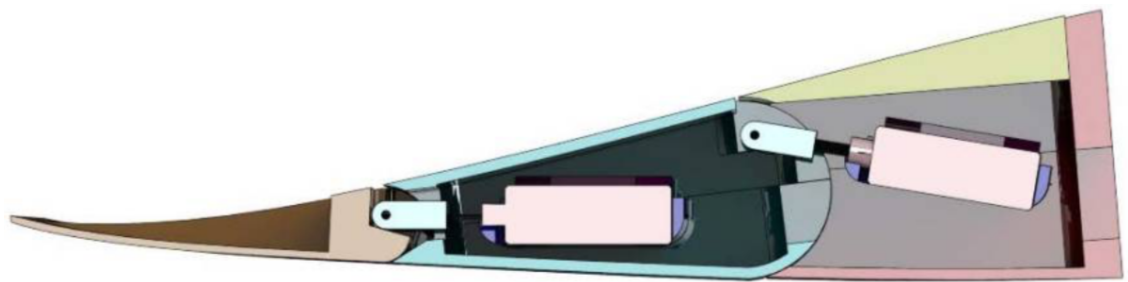

Figure 8. Cutaway View of VCCTEF Actuator Assembly of Flaps 2A and 2B 


\section{B. CRM Wing Design for 2019 Wind Tunnel Test}

Several changes to the CRM wing design are made for the gust load alleviation wind tunnel test in 2019. One of the findings from the 2018 wind tunnel test is that the cradle actuator housing design probably would not provide enough accuracy for active GLA control. Moreover, because of the anticipated increase in the total unsteady gust load that the wing would see, a decision was made to include an aluminum spar design in the foam core construction to provide a higher strength wing structure. This is shown in Fig. 9. The actuator bays that house the servos and the actuator mechanisms are rigidly connected to the spar as opposed to the cradle design. This is shown in Fig. 10.

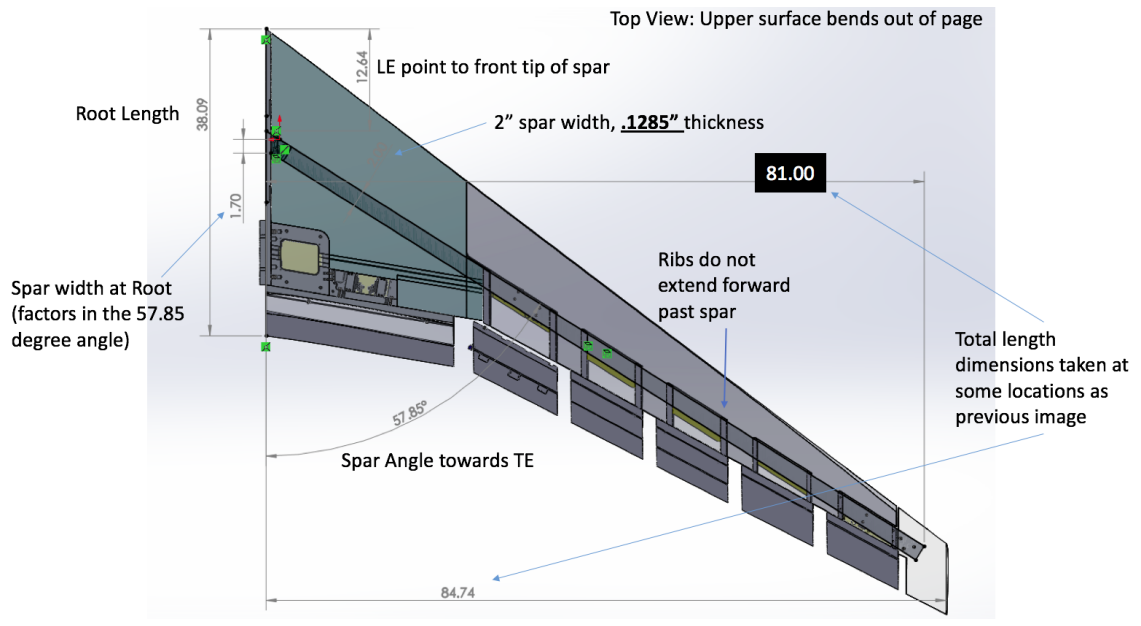

Figure 9. Planform of CRM Wing for Multi-Objective Gust Load Alleviation/Drag Optimization Experiment in 2019

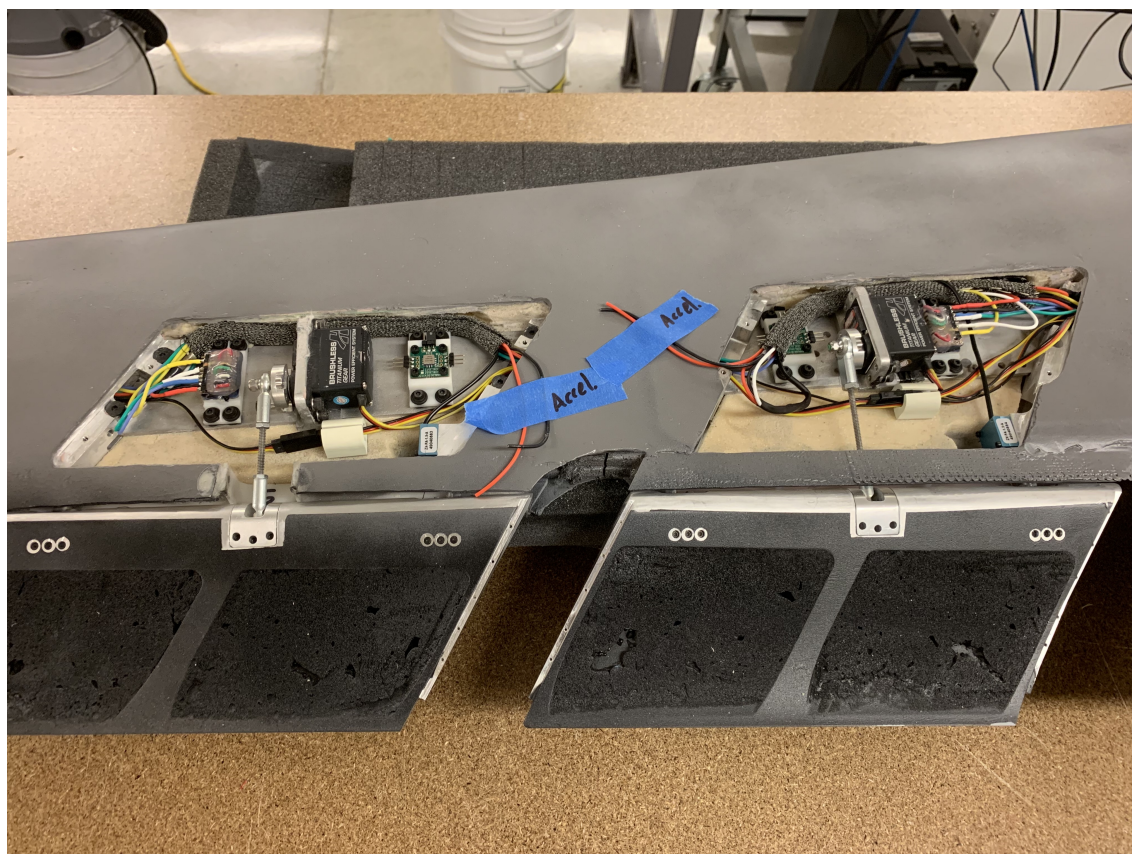

Figure 10. Photo of Underside of CRM Wing Showing Actuator Bays

Because the actuators for the GLA are required to have a higher frequency bandwidth than the actuators in the 2018 real-time drag optimization wind tunnel test, the servos are selected to be more robust and thus are larger in size. Consequently, these actuators create protuberances on the underside of the wing aerodynamic surface which can be 
seen in Fig. 11. Fairings are built to cover these protuberances. The actuators are completely redesigned to meet the new requirement for a higher-frequency response at the frequency of the first bending mode which ranges between 2 $\mathrm{Hz}$ and $3 \mathrm{~Hz}$. The new actuators are designed to have a frequency bandwidth of up to $9 \mathrm{~Hz}$ which gives a sufficient margin for controlling the anticipated response of the first bending mode. To simplify the actuator design, only the two inboard flap sections have two cambered segments for drag optimization. These are flaps 1A, 1B, 2A, and 2B. The other four outboard flap sections only have single segments. A total of eight flap segments are used in the design.

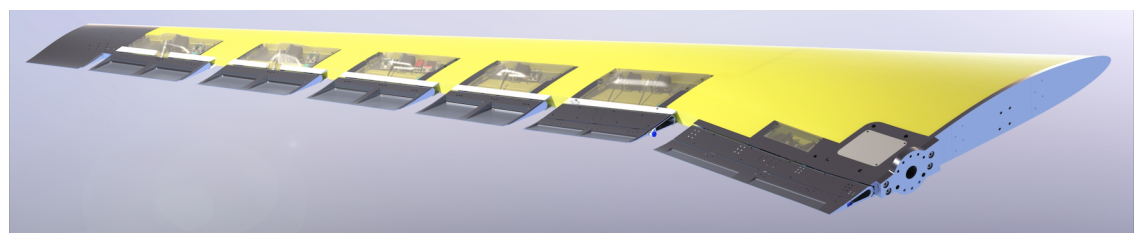

Figure 11. Underside View of CRM Wing Showing Actuator Bays

Figure 12 is the bottom view of the CRM wing showing the locations of the sensors which are co-located in the actuator bays. Figure 10 is a photo showing in detail the servos, actuator linkage mechanisms, the accelerometers. A total of 6 accelerometers and 3 strain gauges are installed in the actuator bays. The accelerometers are to be used to reconstruct the state information for the control law design. The strain gauges provide the load information for the bending moment measurements. This load information is used directly in the control laws as well as to assess the performance of the gust load alleviation.

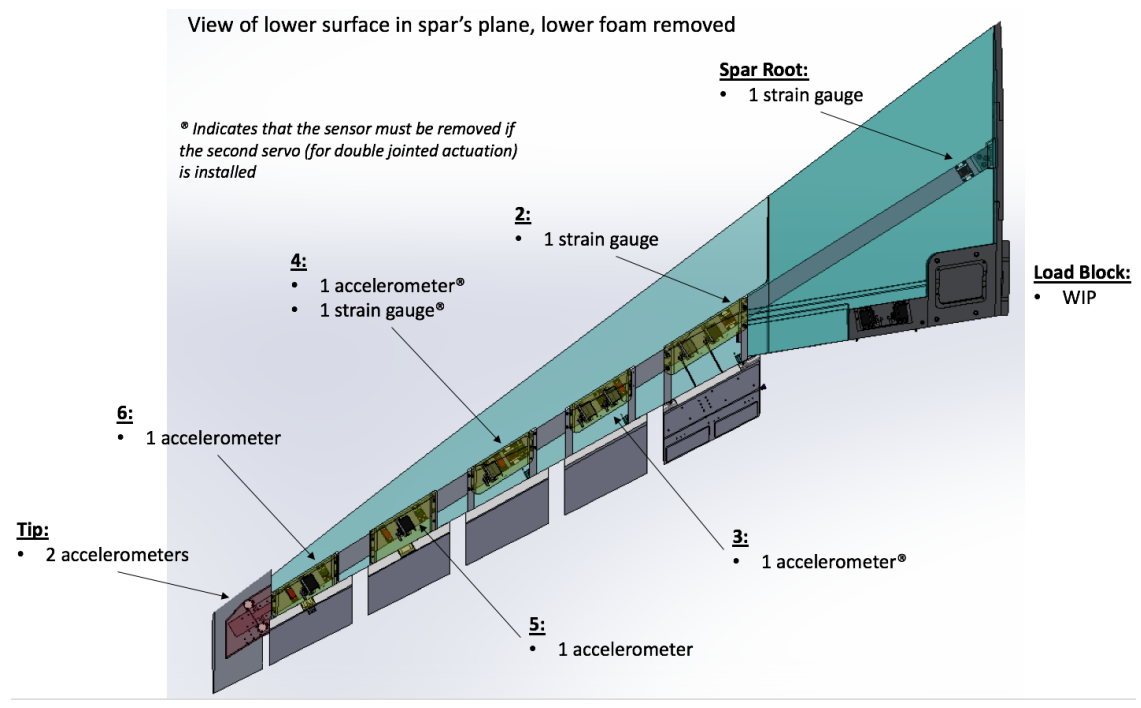

Figure 12. Sensor Locations of CRM Wing

A provision to add a ballast mass to the wing tip is incorporated in the wing design to tailor the nature frequency of the first bending mode, as shown in Fig. 13. The natural frequency of the first bending mode is estimated to be about $2.8 \mathrm{~Hz}$ without a ballast mass. Using a ballast mass weighing $3.625 \mathrm{lbs}$, the natural frequency can be reduced to $2.0 \mathrm{~Hz}$. This reduced frequency will translate into less control power and lower control frequency bandwidth for the actuators which should help improve their operations and increase the performance of the gust load alleviation control laws.

Figure 14 illustrates the architecture of the control system. The real-time control software which is provided by SSCI interfaces with the actuator servos via Arduino micro-controllers. The software controls the actuator servos using a standard PID (proportional-integral-derivative) control law to command the flaps to meet the position requirements computed by the control laws. The software also interfaces with the sensors via a different set of Arduino microcontrollers to process the sensor signals for the control laws. The processed sensor signals are then used to produce the estimates of the state information which in turn is used by the control laws to compute the flap position commands. 


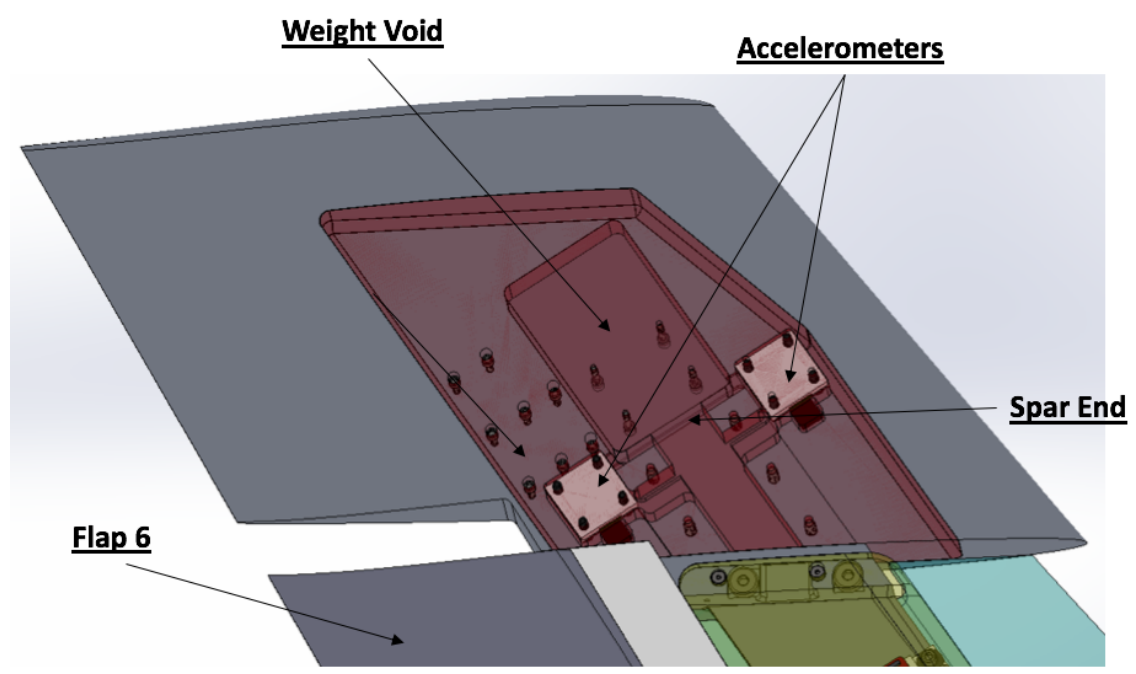

Figure 13. Ballast Mass Provision for Frequency Tailoring

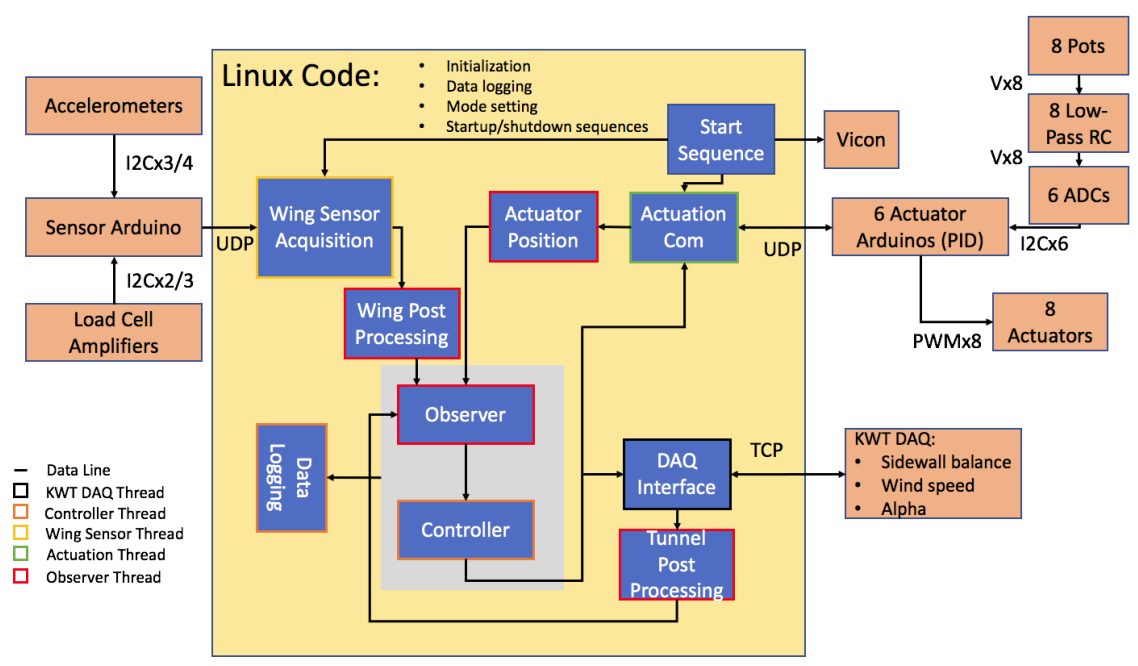

Figure 14. Control Architecture Diagram

\section{Gust Generator}

A gust generator is designed, fabricated, and installed in the KWT at the UW, as shown in Fig. 15. The gust generator comprises four vanes installed upstream of the test section. The vanes are driven by a drive motor to provide the gust excitation to the CRM wing. The vanes have a 15 -inch chord. The center of rotation of the vanes is at $30 \%$ chord. The cross section of the vanes is a NACA 0015 airfoil with a modified trailing edge portion having a straight line connecting the maximum thickness location to the trailing edge location. The vanes are spaced 17 inches apart vertically. The top and bottom vanes are at a distance of 22.5 inches from the ceiling and floor of the test section. The trailing edge plane of the vanes is physically verified to be 14.64 inches ahead of the leading edge of the wing root section. Thus, the wing and the gust vanes are an aerodynamically coupled system. Figure 16shows the dimensions of the gust vanes in relation to the wing airfoil sections. A flow characterization test has been performed to characterize the flow downstream from the vanes using a pressure rake as shown in Fig. 15. 


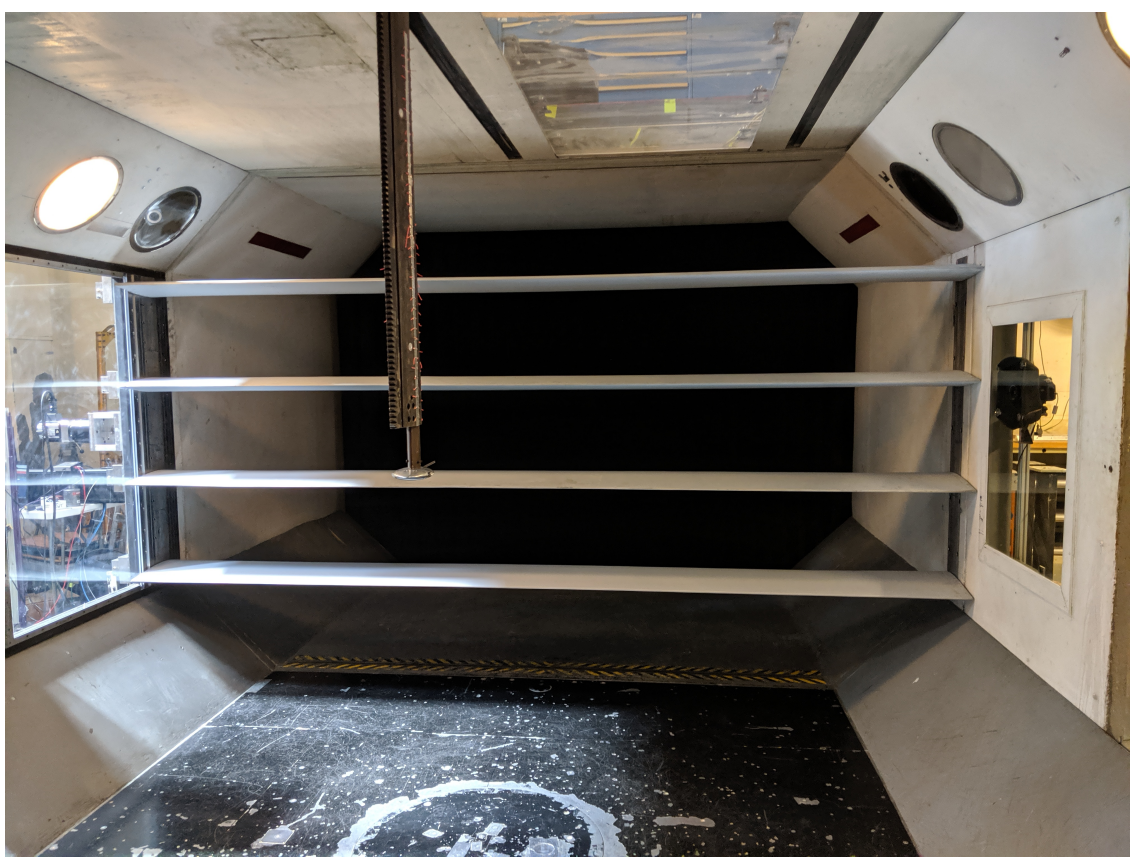

Figure 15. Gust Generator in Kirsten Wind Tunnel at University of Washington

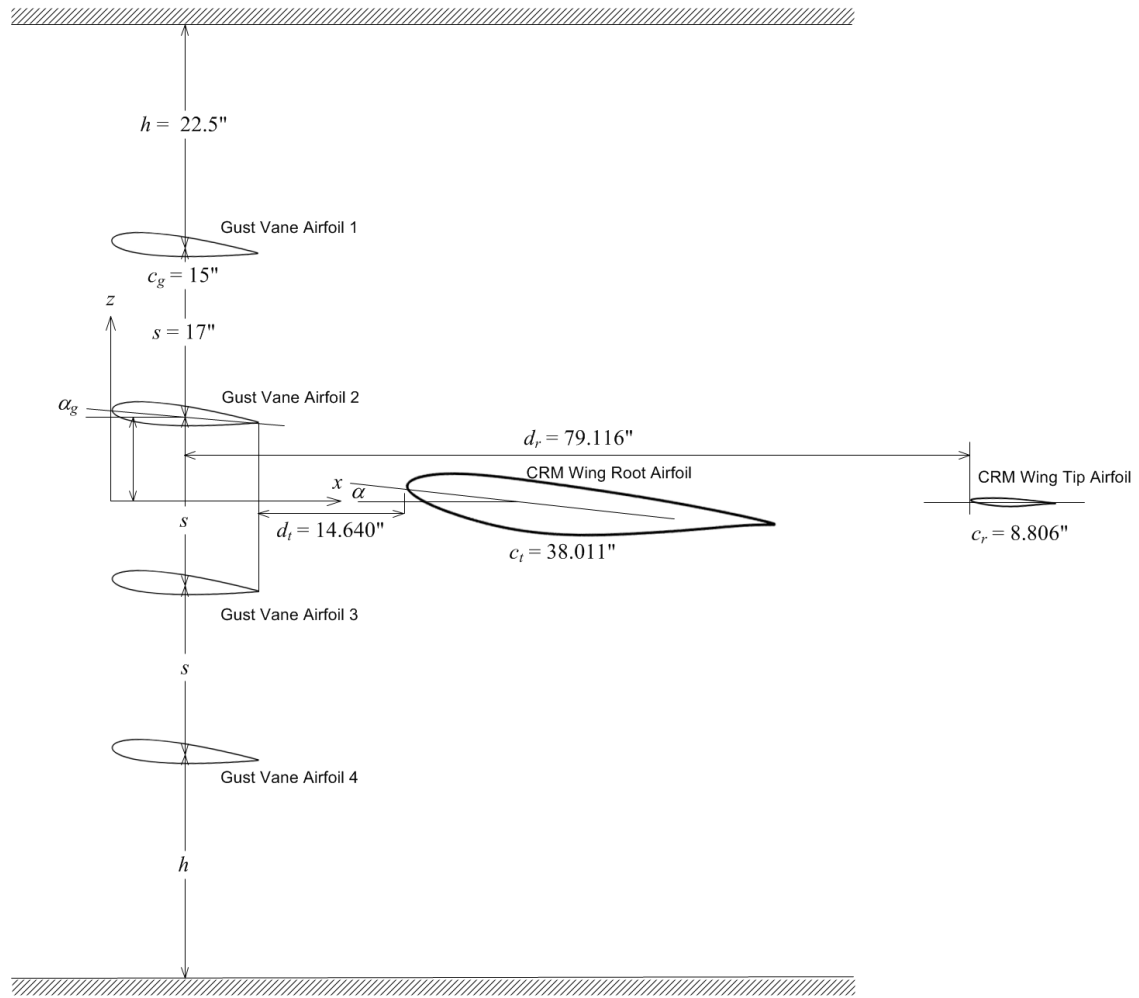

Figure 16. Gust Vane Dimensions in Relation to CRM Wing Airfoil Sections 


\section{Aeroservoelastic Model Development}

NASA develops an initial ASE model based on a beam finite-element model (FEM) of the CRM wing for the initial control design effort. The initial ASE model development can be found in the references. This model was provided to SSCI with the understanding that an improved validated ASE model would be later developed by UW for use in the final control law design. However, this model was still not available at the start of the first test entry which took place on July 15, 2019. Consequently, NASA develops a second ASE model during the test entry with the goal that it would be used to design the final control laws. This model was then provided to SSCI for their control law design. The second ASE model is based on the NASTRAN three-dimensional (3D) FEM of the CRM wing developed by UW. The ASE model uses an elastic axis representation of the NASTRAN mode shapes of the first five modes for which the natural frequencies have been obtained from an impact hammer test.

Table 2 shows the characteristics of the NASTRAN FEM. Figure 17shows the NASTRAN FEM of the CRM wing. The NASTRAN FEM has been tuned to match the first five natural frequencies which are shown in Table 3 . Figure 18 shows the first five mode shapes computed by NASTRAN.

\begin{tabular}{|l|l|}
\hline Number of Structural Elements & 3322 \\
\hline Number of Aerodynamic Elements & 1120 \\
\hline Number of Various Material Properties & 13 \\
\hline Skin and Skin Reinforcements & Membrane Elements with Orthotropic Material \\
\hline Foam, Wing Tip, Reinforcements & 3D Elements \\
\hline Inter-Flap Silicon Elements & Spring Elements \\
\hline Aluminum Pass & Beam Elements \\
\hline
\end{tabular}

Table 2. UW NASTRAN FEM Characteristics

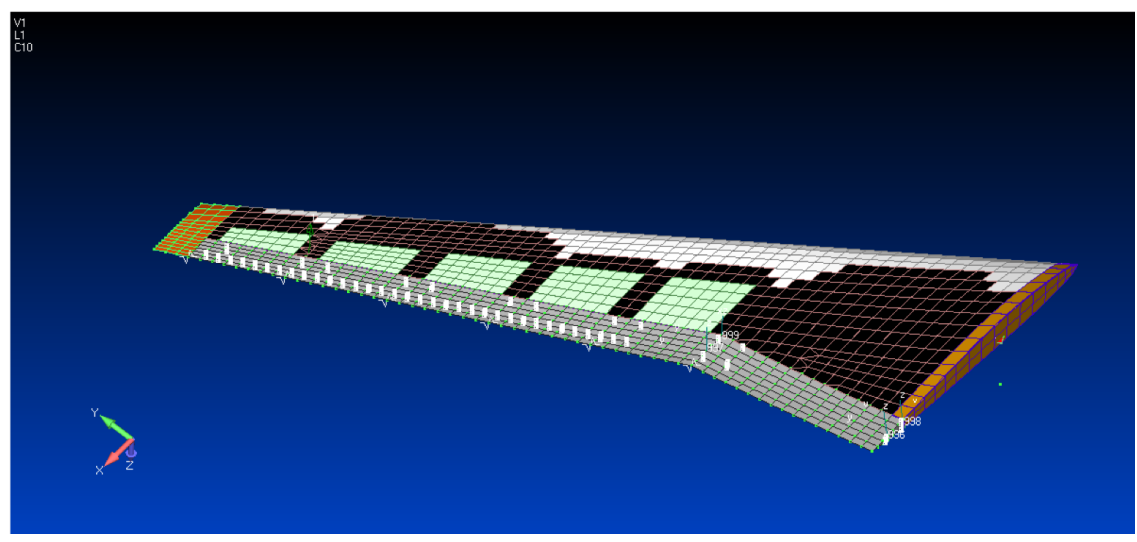

Figure 17. UW NASTRAN FEM of CRM Wing Showing the Lower Surface

\begin{tabular}{|c|c|c|c|}
\hline Mode & Description & $\begin{array}{c}\text { Experimental } \\
\text { Natural Frequency (Hz) }\end{array}$ & $\begin{array}{c}\text { NASTRAN } \\
\text { Natural Frequency (Hz) }\end{array}$ \\
\hline 1 & $1^{\text {st }}$ Bending & 2.2 & 2.2 \\
\hline 2 & $1^{\text {st }}$ In-Plane Bending & 9.4 & 9.4 \\
\hline 3 & $2^{\text {nd }}$ Bending & $10-11$ & 10.8 \\
\hline 4 & $3^{\text {rd }}$ Bending & 23 & 24 \\
\hline 5 & $1^{\text {st }}$ Torsion & 30 & 29 \\
\hline
\end{tabular}

Table 3. Natural Frequencies of CRM Wing 

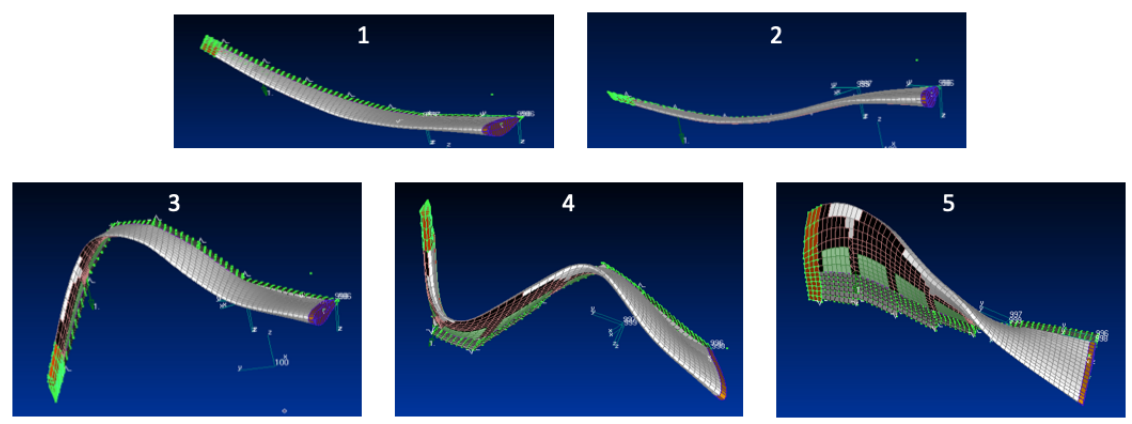

Figure 18. NASTRAN Mode Shapes

The 3D NASTRAN mode shapes are transformed into the equivalent beam mode shapes in bending and torsion about an axis that approximates the elastic axis, as shown in Fig. 19. Since the in-plane bending mode is not likely to be excited in the wind tunnel, the in-plane bending mode shape is eliminated. Figures 20 and 21 show the normalized bending and torsion mode shapes of the first four modes without the in-plane bending mode. Note that the bending displacement is not zero at the wing root. This is due to the inclusion of the side wall balance adapter tube attached to the wing root block in the NASTRAN FEM.

The generalized masses and stiffnesses of the four modes are extracted from the NASTRAN FEM. These generalized quantities are mass-normalized to a value of 1 and have no physical meaning. To model the static deflection of the wing, the generalized stiffness must be scaled appropriately. A scaling factor is derived to match the computed static deflection using all the four mode shapes to the measured static deflection provided by the VICON system in the wind tunnel. In order to do this, the lift force acting on the wing must be known. Then, using an assumed elliptical lift distribution, the generalized force is computed. Using the NASTRAN generalized stiffness matrix, the static deflection can be computed. This static deflection is then compared to the known static deflection to determine a stiffness scaling factor to be applied to the generalized masses and stiffnesses. This scaling procedure is applied to one specific test condition at $C_{L}=0.4$ which corresponds to about $48 \mathrm{lbs}$ of lift at a dynamic pressure of $10 \mathrm{psf}$. The wing tip deflection is obtained from the 2018 wind tunnel test and is about 6 inches.

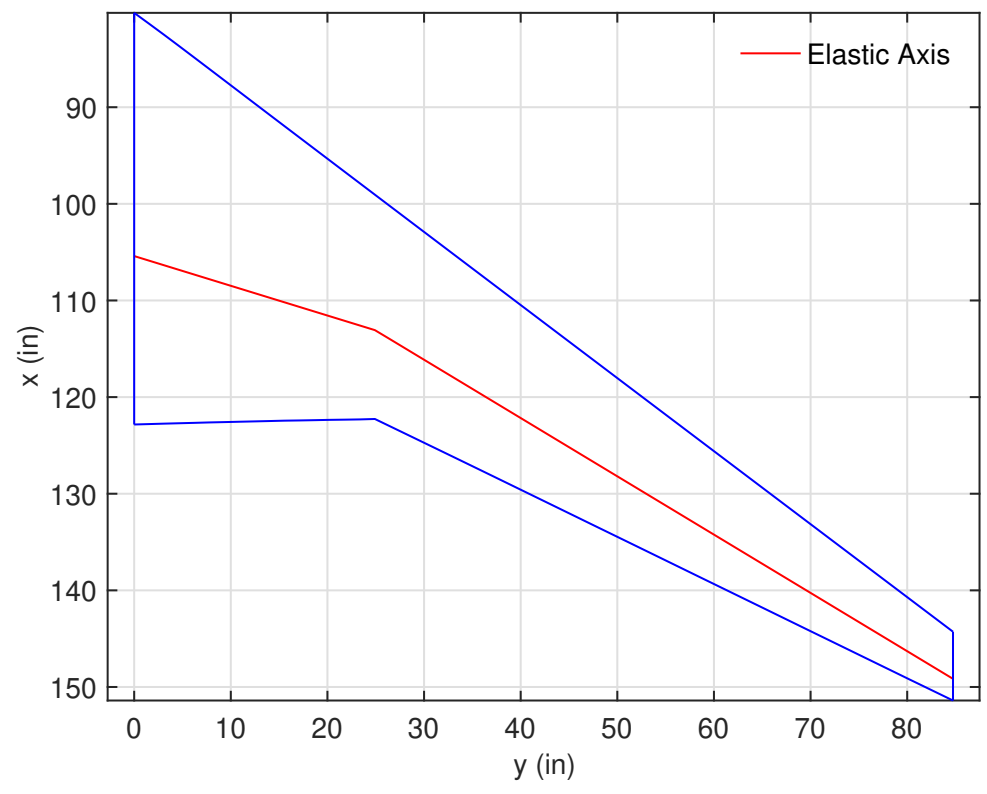

Figure 19. Elastic Axis Representation of CRM Wing 


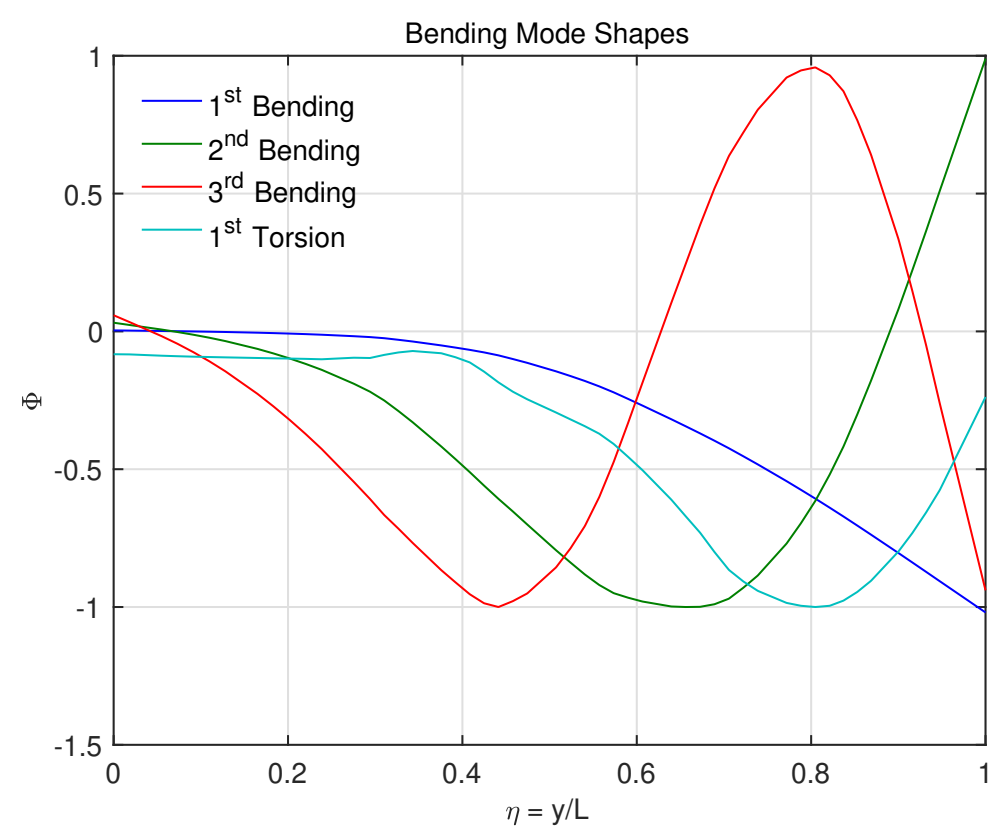

Figure 20. NASTRAN Bending Mode Shapes

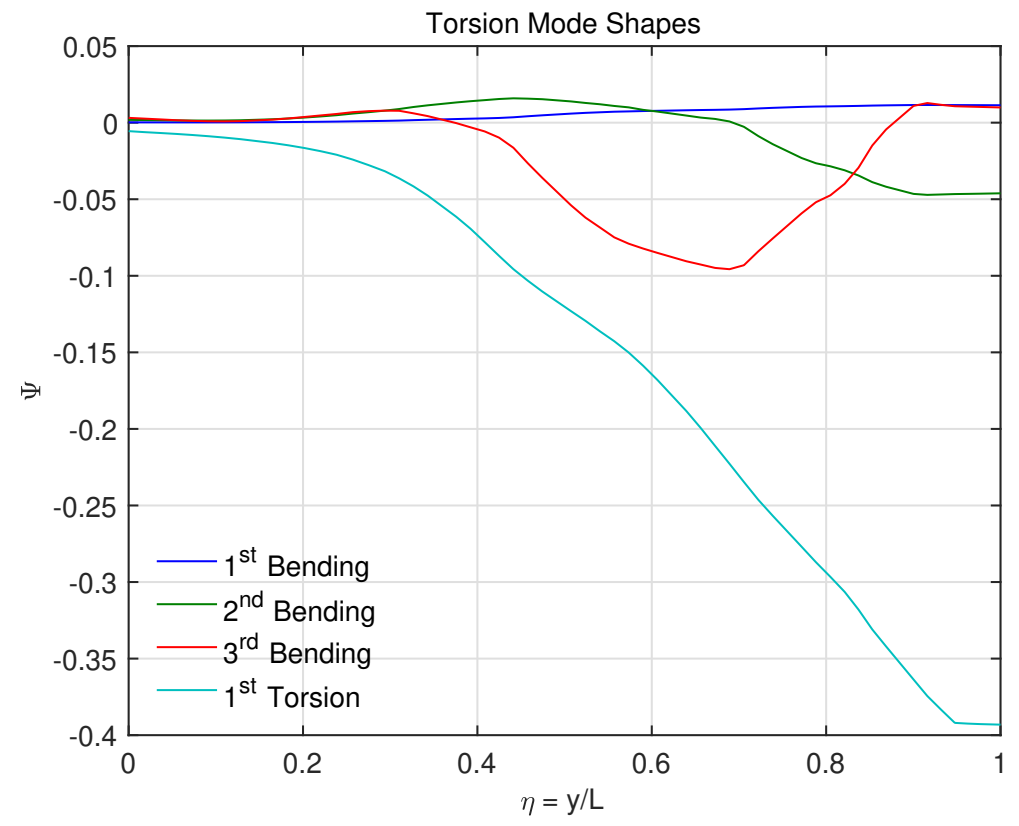

Figure 21. NASTRAN Torsion Mode Shapes

The ASE model comes down to determining the generalized aerodynamic, control, and gust forces. The wing is excited by the gust generator in a low frequency range well below the $9 \mathrm{~Hz}$ bandwidth of the actuators. So the most dominant mode is the $1^{\text {st }}$ bending mode. Therefore, the ASE model is designed to capture only the bending motion of the wing, but the torsional contribution to the bending motion is fully accounted for.

The CRM wing is modeled as a beam using the standard bending-torsion aeroelastic equations

$$
\rho A \frac{\partial^{2} w}{\partial t^{2}}+\rho A e_{c g} \frac{\partial^{2} \theta}{\partial t^{2}}+\frac{\partial^{2}}{\partial x^{2}}\left(E I_{y y} \frac{\partial^{2} w}{\partial x^{2}}\right)=\bar{l} \cos \Lambda+\frac{\partial \bar{m}}{\partial x} \cos \Lambda \sin \Lambda
$$




$$
\rho I_{x x} \frac{\partial^{2} \theta}{\partial t^{2}}+\rho A e_{c g} \frac{\partial^{2} w}{\partial t^{2}}-\frac{\partial}{\partial x}\left(G J \frac{\partial \theta}{\partial x}\right)=\bar{m} \cos ^{2} \Lambda
$$

where $w(x, t)$ is the bending vertical displacement, $\theta(x, t)$ is the torsional twist angle, $e_{c g}$ is the offset of the center of mass from the elastic axis, positive when the center of mass lies forward of the elastic axis, and $\bar{l}(x, t)$ and $\bar{m}(x, t)$ are the aerodynamic lift and pitching moment about the pitch axis respectively in the streamwise direction due to the wing aeroelasticity, flap motion, and gust disturbance. They can be written as

$$
\begin{gathered}
\bar{l}=\bar{l}_{a}+\bar{l}_{c}+\bar{l}_{g} \\
\bar{m}=\bar{m}_{a}+\bar{m}_{c}+\bar{m}_{g}
\end{gathered}
$$

The bending vertical displacement and torsional twist angle can be expressed in terms of the generalized displacement $q(t) \in \mathbb{R}^{n}$ as

$$
\left[\begin{array}{c}
w \\
\theta
\end{array}\right]=\left[\begin{array}{l}
\Phi \\
\Psi
\end{array}\right] q
$$

where $\Phi(x) \in \mathbb{R}^{1 \times n}$ is the bending mode shape matrix and $\Psi(x) \in \mathbb{R}^{1 \times n}$ is the torsional mode shape matrix.

\section{A. Generalized Aerodynamic Forces Due to Wing Aeroelasticity}

The aerodynamic lift and pitching moment about the elastic axis of a wing section in the streamwise direction are given by

$$
\begin{aligned}
& \bar{l}_{a}=q_{\infty} c\left(c_{L_{0}}+c_{L_{\alpha}} \alpha\right)+C(k) q_{\infty} c c_{L_{\alpha}}\left(\theta \cos \Lambda-\frac{\partial w}{\partial x} \sin \Lambda\right.\left.+\frac{e_{c}}{V_{\infty}} \frac{\partial \theta}{\partial t} \cos \Lambda-\frac{1}{V_{\infty}} \frac{\partial w}{\partial t}\right) \\
&+\frac{\pi}{4} \rho_{\infty} c^{2}\left(V_{\infty} \frac{\partial \theta}{\partial t} \cos \Lambda+e_{m} \frac{\partial^{2} \theta}{\partial t^{2}} \cos \Lambda-\frac{\partial^{2} w}{\partial t^{2}}\right) \\
& \bar{m}_{a}=q_{\infty} c\left(c c_{m_{a c}}+e c_{L_{0}}+e c_{L_{\alpha}} \alpha\right)+C(k) q_{\infty} c e c_{L_{\alpha}}\left(\theta \cos \Lambda-\frac{\partial w}{\partial x} \sin \Lambda+\frac{e_{c}}{V_{\infty}} \frac{\partial \theta}{\partial t} \cos \Lambda-\frac{1}{V_{\infty}} \frac{\partial w}{\partial t}\right) \\
&-\frac{\pi}{4} \rho_{\infty} c^{2} e_{m}\left(e_{m} \frac{\partial^{2} \theta}{\partial t^{2}} \cos \Lambda-\frac{\partial^{2} w}{\partial t^{2}}\right)-\frac{\pi}{4} \rho_{\infty} c^{2} e_{c} V_{\infty} \frac{\partial \theta}{\partial t} \cos \Lambda-\frac{\pi}{128} \rho_{\infty} c^{4} \frac{\partial^{2} \theta}{\partial t^{2}} \cos \Lambda
\end{aligned}
$$

where $\Lambda$ is the wing sweep angle of the elastic axis, $c$ is the wing chord, $e$ is the offset of the elastic center from the quarter chord, $e_{m}$ is the offset of the mid chord from the elastic center, $e_{c}$ is the offset of the three-quarter chord from the elastic center, $q_{\infty}$ is the dynamic pressure, $V_{\infty}$ is the airspeed, $\rho_{\infty}$ is the air density, $c_{L_{\alpha}}$ is the lift curve slope, $C(k)$ is the Theodorsen's function of the reduced frequency $k=\frac{\omega \bar{c}}{2 V_{\infty}}, \omega$ is the frequency of oscillation, $\bar{c}$ is the mean aerodynamic chord, and $V_{\infty}$ is the airspeed. The section lift curve slope $c_{L_{\alpha}}$ is assumed to be the same as the wing lift curve slope $C_{L_{\alpha}}$ determined from the lift curve measurements.

The aerodynamic lift and pitching moment can be expressed in terms of the generalized displacement as

$$
\begin{gathered}
l_{a}=\bar{l}_{a} \cos \Lambda=l_{0}+l_{\alpha} \alpha+C(k) l_{q}^{*} q+C(k) l_{\dot{q}}^{*} \dot{q}+l_{\dot{q}} \dot{q}+l_{\ddot{q}} \ddot{q} \\
m_{a}=\bar{m}_{a} \cos \Lambda \sin \Lambda=m_{0}+m_{\alpha} \alpha+C(k) m_{q}^{*} q+C(k) m_{\dot{q}}^{*} \dot{q}+m_{\dot{q}} \dot{q}+m_{\ddot{q}} \ddot{q}
\end{gathered}
$$

where the lift and pitching moment sensitivities are provided in Appendix A1. The asterisk superscript denotes the circulatory quantities which are multiplied by the Theodorsen's function which is applicable for the test conditions below Mach 0.1 .

The generalized aerodynamic force due to wing aeroelasticity is computed using integration by parts as

$$
F_{a}=\int_{0}^{L}\left(\Phi^{\top} l_{a}+\Phi^{\top} \frac{\partial m_{a}}{\partial x}\right) d x=\int_{0}^{L}\left(\Phi^{\top} l_{a}-\Phi^{\top} m_{a}\right) d x
$$

which can be expressed as

$$
F_{a}=F_{0}+F_{\alpha} \alpha-M_{a} \ddot{q}-C_{a} \dot{q}-C(k) C_{a}^{*} \dot{q}-C(k) K_{a}^{*} q
$$


where the generalized aerodynamic force sensitivities are provided in Appendix A2.

The total lift of the wing is computed as

$$
\begin{gathered}
L_{a}=q_{\infty} S\left(C_{L_{0}}+C_{L_{\alpha}} \alpha\right)+C(k) L_{q}^{*} q+C(k) L_{\dot{q}}^{*} \dot{q}+L_{\dot{q}} \dot{q}+L_{\ddot{q}} \ddot{q} \\
M_{a}=q_{\infty} S \bar{c}\left(C_{m_{0}}+C_{m_{\alpha}} \alpha\right)+C(k) M_{q}^{*} q+C(k) M_{\dot{q}}^{*} \dot{q}+M_{\dot{q}} \dot{q}+M_{\ddot{q}} \ddot{q}
\end{gathered}
$$

where the lift and pitching moment sensitivities are provided in Appendix A3.

\section{B. Generalized Aerodynamic Forces Due to Flap Motion}

The lift and pitching moment of a flap section in the streamwise direction are given by

$$
\begin{gathered}
\bar{l}_{c}=q_{\infty} c c_{L_{\delta}} \bar{\delta}+C(k) q_{\infty} c\left(c_{L_{\delta}} \delta+c_{L_{\dot{\delta}}} \frac{c \dot{\delta}}{2 V_{\infty}}\right)+q_{\infty} c\left(\bar{c}_{L_{\dot{\delta}}} \frac{c \dot{\delta}}{2 V_{\infty}}+\bar{c}_{L_{\ddot{\delta}}} \frac{c^{2} \ddot{\delta}}{4 V_{\infty}^{2}}\right) \\
\bar{m}_{c}=q_{\infty} c\left(c c_{m_{\delta}}+e c_{L_{\delta}}\right) \bar{\delta}+C(k) q_{\infty} c e\left(c_{L_{\delta}} \delta+c_{L_{\dot{\delta}}} \frac{c \dot{\delta}}{2 V_{\infty}}\right)+q_{\infty} c^{2}\left(c_{m_{\delta}} \delta+c_{m_{\dot{\delta}}} \frac{c \dot{\delta}}{2 V_{\infty}}+c_{m_{\ddot{\delta}}} \frac{c^{2} \ddot{\delta}}{4 V_{\infty}^{2}}\right)
\end{gathered}
$$

where $\delta$ is the flap deflection in the streamwise direction, positive downward, $\bar{\delta}$ is the steady state flap deflection, and the lift and pitching moment coefficient sensitivities due to the flap motion are provided in Appendix A4.

Let

$$
\begin{gathered}
l_{c}=\bar{l}_{c} \cos \Lambda=l_{\bar{\delta}} \bar{\delta}+C(k) l_{\delta}^{*} \delta+C(k) l_{\dot{\delta}}^{*} \dot{\delta}+l_{\dot{\delta}} \dot{\delta}+l_{\ddot{\delta}} \ddot{\delta} \\
m_{c}=\bar{m}_{c} \cos \Lambda \sin \Lambda=l_{\bar{\delta}} \bar{\delta}+C(k) m_{\delta}^{*} \delta+C(k) m_{\dot{\delta}}^{*} \dot{\delta}+m_{\delta} \delta+m_{\dot{\delta}} \dot{\delta}+m_{\ddot{\delta}} \ddot{\delta}
\end{gathered}
$$

where the lift and pitching moment sensitivities due to the flap motion are provided in Appendix A5.

The generalized aerodynamic force due to the flap motion is computed as

$$
F_{c}=\int_{0}^{L}\left(\Phi^{\top} l_{c}+\Phi^{\prime} \frac{\partial m_{c}}{\partial x}\right) d x=\int_{0}^{L}\left(\Phi^{\top} l_{c}-\Phi^{\prime} m_{c}\right) d x
$$

which can be expressed as

$$
F_{c}=F_{\delta} \bar{\delta}+C(k) F_{\delta}^{*} \delta+F_{\dot{\delta}} \dot{\delta}+C(k) F_{\dot{\delta}}^{*} \dot{\delta}+F_{\ddot{\delta}} \ddot{\delta}
$$

where the generalized aerodynamic force sensitivities due to the flap motion are given in Appendix A6.

The total integrated lift and pitching moment about the pitch axis due to the flap motion are

$$
\begin{gathered}
L_{c}=q_{\infty} S C_{L_{\delta}} \bar{\delta}+C(k) L_{\delta}^{*} \delta+C(k) L_{\dot{\delta}}^{*} \dot{\delta}+L_{\dot{\delta}} \dot{\delta}+L_{\ddot{\delta}} \ddot{\delta} \\
M_{c}=q_{\infty} S \bar{c}\left(C_{m_{\delta}}+\frac{\bar{e}_{\delta}}{\bar{c}} C_{L_{\delta}}\right) \bar{\delta}+C(k) M_{\delta}^{*} \delta+C(k) M_{\dot{\delta}}^{*} \dot{\delta}+M_{\delta} \delta+M_{\dot{\delta}} \dot{\delta}+M_{\ddot{\delta}} \ddot{\delta}
\end{gathered}
$$

where the total lift and pitching moment sensitivities due to the flap motion are provided in Appendix A7.

The aerodynamic model of the flaps is validated against limited wind tunnel data from the 2018 wind tunnel test. During this test, the lift coefficient sensitivities due to the flaps were estimated. The computed lift coefficient sensitivities are then scaled to match the wind tunnel data. The scaling factors are then applied across the board for all the lift and pitching moment coefficient derivatives shown in the appendix. Table 4 shows the scaling factors for the flaps. In general, the model predicts the lift coefficient sensitivities very well for Flaps 3-6. It tends to over-predict the lift coefficient sensitivities for the inboard flaps.

\begin{tabular}{|c|c|c|c|c|c|c|c|c|}
\hline Flap & $1 \mathrm{~A}$ & $1 \mathrm{~B}$ & $2 \mathrm{~A}$ & $2 \mathrm{~B}$ & 3 & 4 & 5 & 6 \\
\hline Scaling Factor & 0.7401 & 0.7401 & 0.7204 & 0.7204 & 1.0370 & 0.9159 & 0.8866 & 0.9321 \\
\hline
\end{tabular}

Table 4. Scaling Factors for Aerodynamic Coefficient Sensitivities Due to Flap Motion 


\section{Generalized Aerodynamic Forces due to Gust}

The gust generator can generate two types of gust signals: sinusoidal and one-minus cosine. Because of the aerodynamic coupling between the gust vanes and the wing, the standard method of estimating the gust load may not be accurate. Thus, CFD is used to model the unsteady flow between the gust vanes and the wing sections. Details of the CFD model of the gust generator-wing system is provided in the references. Only sinusoidal gust CFD simulations are performed at various frequencies and amplitudes of oscillation of the gust vanes.

\section{Gust Modeling and Simulations}

A RANS (Reynolds-Averaged N-S) CFD model of the gust generator is developed using OpenFOAM CFD solver. Figure 22 illustrates the pressure distribution of the flow field created by an oscillation of the gust vanes. CFD models of the gust vanes alone and with three CRM wing airfoils at the root, MAC, and tip are created. Simulations are conducted for $1 \mathrm{~Hz}, 1.8 \mathrm{~Hz}$, and $2 \mathrm{~Hz}$ frequencies of the gust vanes at $4^{\circ}, 5^{\circ}$, and $10^{\circ}$ amplitudes.

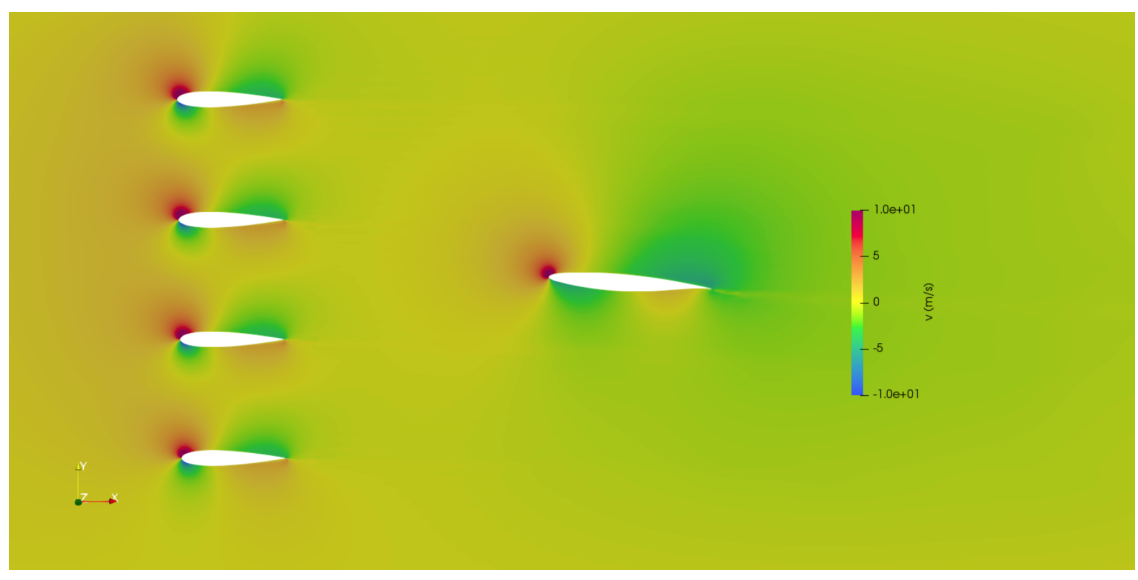

Figure 22. CFD Model of Gust Vanes and CRM Wing Section at MAC

To estimate the unsteady lift on a wing section, the wake velocity field is used to determine the instantaneous gust angle of attack at the wing leading edge due to the gust vane motion. Because the wing section creates a local upwash at the leading edge, it is difficult to determine the unperturbed gust angle of attack. Therefore, the wake velocity field for the CFD model of the gust vanes alone is used. Two quantities are extracted from the CFD simulation data, the aerodynamic lag and the amplitude of the gust angle of attack at the wing leading edge.

The gust vane pitch angle command is described by

$$
\theta_{v}=-\theta_{0} \sin \omega t
$$

where the negative angle indicates a leading edge down pitch rotation. This generates a gust angle of attack downstream the gust vane trailing edge described by

$$
\alpha_{g}=r \theta_{v}\left(t-t_{d}\right)=r \theta_{0} \sin \omega\left(t-t_{d}\right)=-r \cos \omega t_{d} \theta_{v}+r \sin \omega t_{d} \frac{\dot{\theta}_{v}}{\omega}
$$

where $r$ is the ratio of the gust angle of attack to the gust vane amplitude and $t_{d}$ is the gust aerodynamic lag.

Figures 23 and 24 show the ratio of the gust angle of attack to the gust vane amplitude and the gust aerodynamic lag, respectively, as functions of the distance downstream the gust vanes measured from the gust vane trailing edge normalized to the gust vane chord $c_{g}=15$ in at zero pitch angle for $\theta_{0}=5^{\circ}$ and $\omega=2 \mathrm{~Hz}$. This frequency is selected to be the excitation frequency. The $5^{\circ}$ gust amplitude has been verified experimentally to produce reasonable unsteady lift on the wing. It can be seen that at a distance $6 c_{g}$ the ratio decreases to about 0.3 and remains nearly constant. For reference, the wing root and tip sections are at distances of $0.98 c_{g}$ and $5.27 c_{g}$, respectively. 


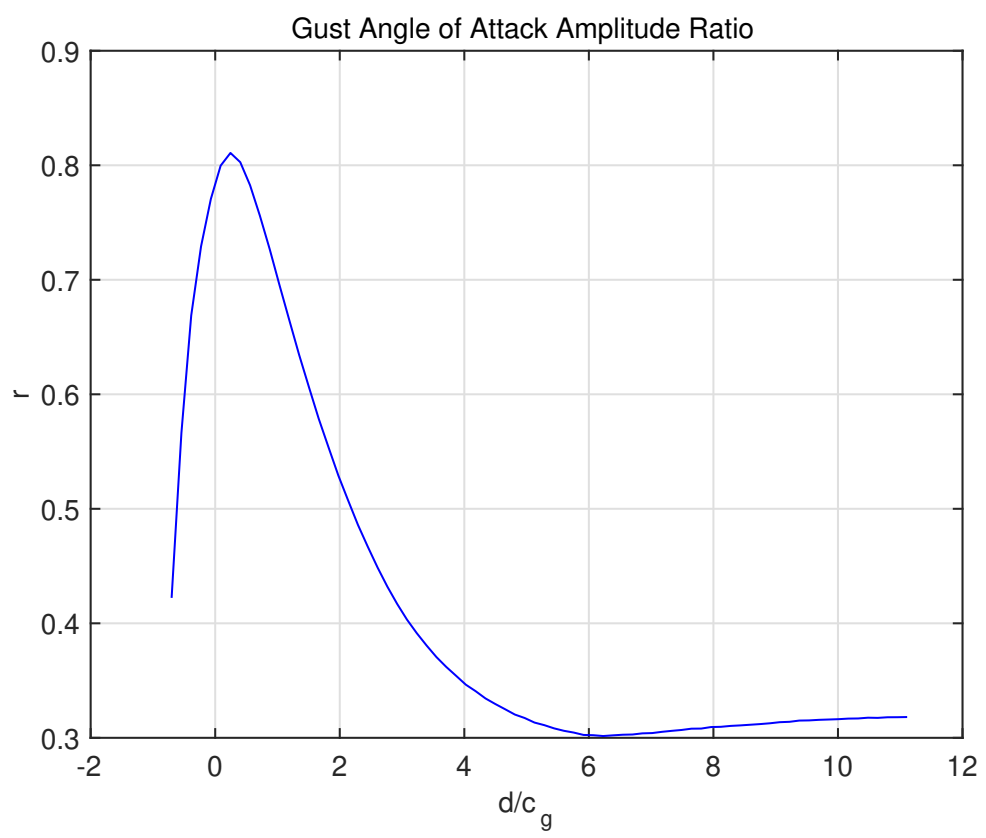

Figure 23. Ratio of Gust Angle of Attack to Gust Vane Amplitude for $5^{\circ}$ Oscillation at $2 \mathrm{~Hz}$

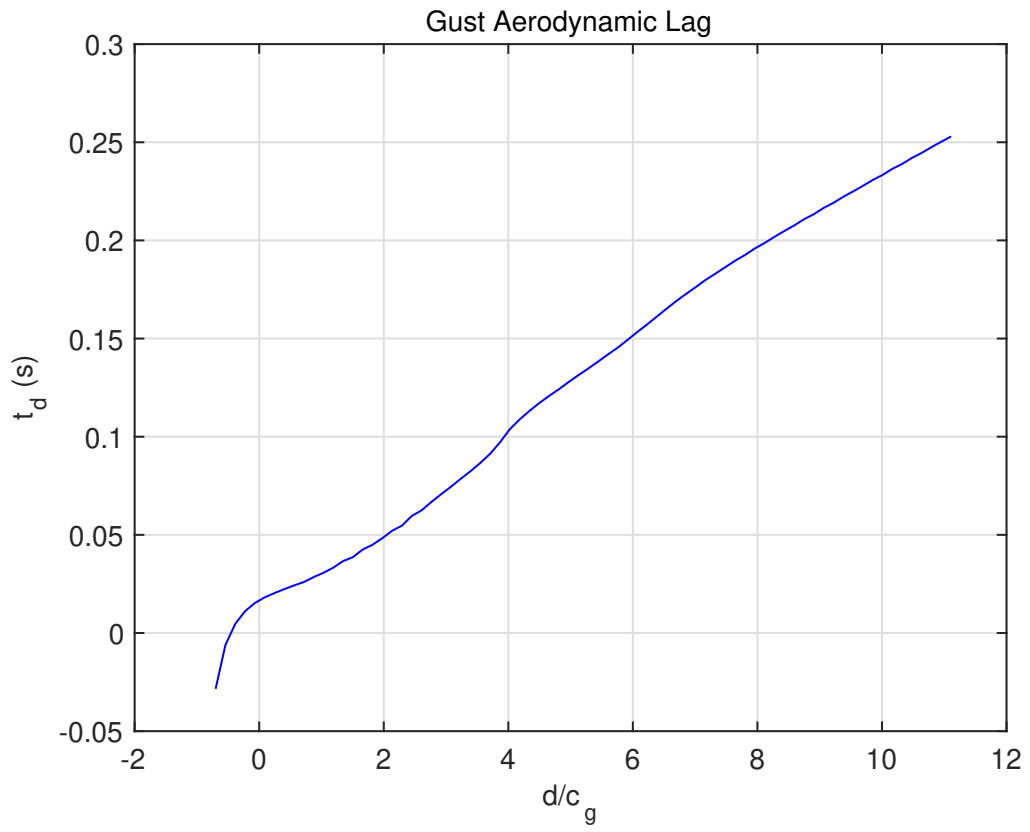

Figure 24. Gust Aerodynamic Lag for $5^{\circ}$ Oscillation at $2 \mathrm{~Hz}$

An analytical vortex model of the gust generator-wing system is also developed using the image method to model the aerodynamic interference between the gust vanes and the wing. The image method based on the aerodynamic conformal mapping creates images of the gust vanes by reflecting the gust vanes about the two solid walls of the test section, as shown in Fig. 25. Thus, the total model includes four gust vanes on top and on the bottom mirrored about the two solid walls and the wing airfoil. Only two images of the two gust vanes next to the walls are shown in Fig. 25.

The lumped vortex model models the flow around the airfoil using a vortex placed at the quarter chord of the airfoil 
and the induced velocity at the three-quarter chord using the Bio-Savart law ${ }^{25}$

$$
\Delta \mathbf{v}=\frac{\Gamma}{4 \pi} \frac{d \mathbf{l} \times\left(\mathbf{r}_{0}-\mathbf{r}_{1}\right)}{\left|\mathbf{r}_{0}-\mathbf{r}_{1}\right|^{3}}
$$

where $\Gamma$ is the circulation, $d \mathbf{l}$ is the differential vortex filament, and $\mathbf{r}$ is a position vector.

The induced axial and vertical velocity components of the $i$-th airfoil on the $j$-th airfoil are computed by

$$
\begin{gathered}
u_{i j}=\frac{\Gamma_{j}}{c_{L_{\alpha_{j}}}} \frac{z_{i}-z_{0, j}}{\left(x_{i}-x_{0, j}\right)^{2}+\left(z_{i}-z_{0, j}\right)^{2}} \\
w_{i j}=-\frac{\Gamma_{j}}{c_{L_{\alpha_{j}}}} \frac{x_{i}-x_{0, j}}{\left(x_{i}-x_{0, j}\right)^{2}+\left(z_{i}-z_{0, j}\right)^{2}}
\end{gathered}
$$

where $\left(x_{i}, z_{i}\right)$ is the three-quarter chord coordinate of the $i$-th airfoil and $\left(x_{0, j}, z_{0, j}\right)$ is the quarter-chord coordinate of the $j$-th airfoil.

The boundary condition of zero normal velocity requires

$$
\sum_{j=1}^{N} u_{i j} \sin \alpha_{i}+w_{i j} \cos \alpha_{i}+V_{\infty}\left(\alpha_{i}-\alpha_{0, i}\right)=0
$$

for the gust vanes and wing airfoil, and

$$
\sum_{j=1}^{N} u_{i j} \sin \alpha_{i}+w_{i j} \cos \alpha_{i}=0
$$

for the image airfoils, where $\alpha_{i}$ is the angle of attack and $\alpha_{0, i}$ is the zero-lift angle of attack of the $i$-th airfoil.

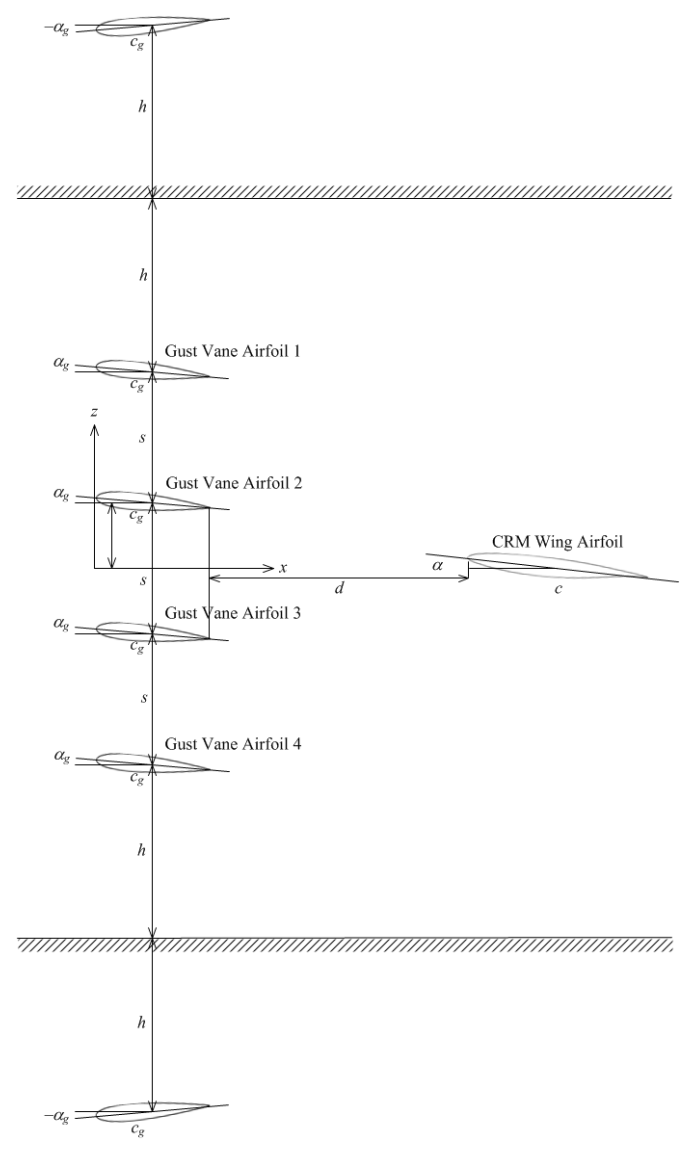

Figure 25. Vortex Model of Gust Generator-Wing System

18 of 43

American Institute of Aeronautics and Astronautics 
Figure 26 shows the lift curves for the four gust vanes alone and with the three CRM wing airfoils at the tip, Yehudi break corresponding to the MAC, and root stations. The lift curve for the gust vane alone is compared to the data computed by OpenFOAM. The two set of results show a similar trend. Gust vane 4 next to the floor has the highest positive lift and gust vane 1 next to the ceiling has the most negative lift. We note the nonlinear behavior in the lift curves. The average lift curve slope for the gust vane is $c_{L_{\alpha, v}}=5.0216$. OpenFOAM gives $c_{L_{\alpha, v}}=5.2894$. We note that as the wing moves closer to the gust vanes, the lifts on the gust vane decrease, but the amount of lift reduction does not appear to be significant especially when the gust vanes are coupled to the wing root airfoil. However, the average lift curve slope for the gust vanes coupled to the wing root airfoil is estimated to be $c_{L_{\alpha, v}}=4.1698$. This represents a $17 \%$ decrease in the lift curve slope. Figure 27 shows the lift curves for the CRM wing airfoils at the root, MAC, and tip coupled to the gust vanes at $c_{L}=0$. As can be seen, the lift of the wing root is less than that of the wing tip due to the aerodynamic interference of the gust vanes. The lift reduction for the wing root airfoil is about $11 \%$. Table 5 lists the lift curve slope values for different gust generator - wing configurations.
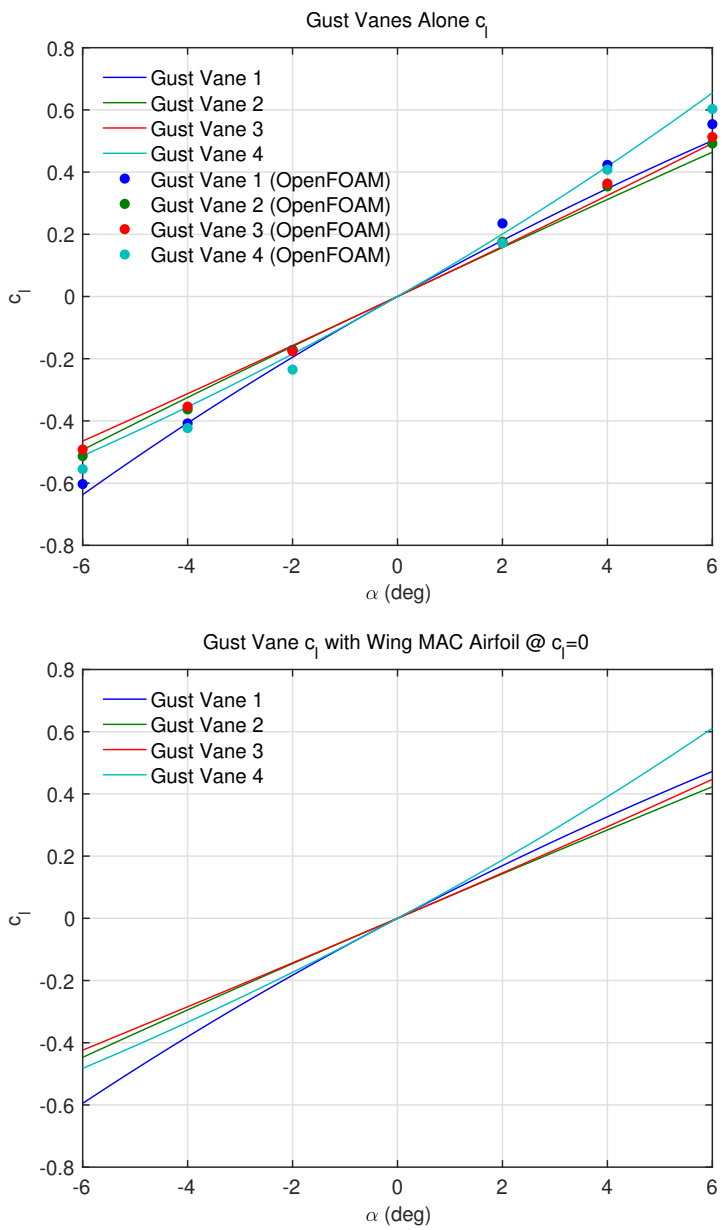
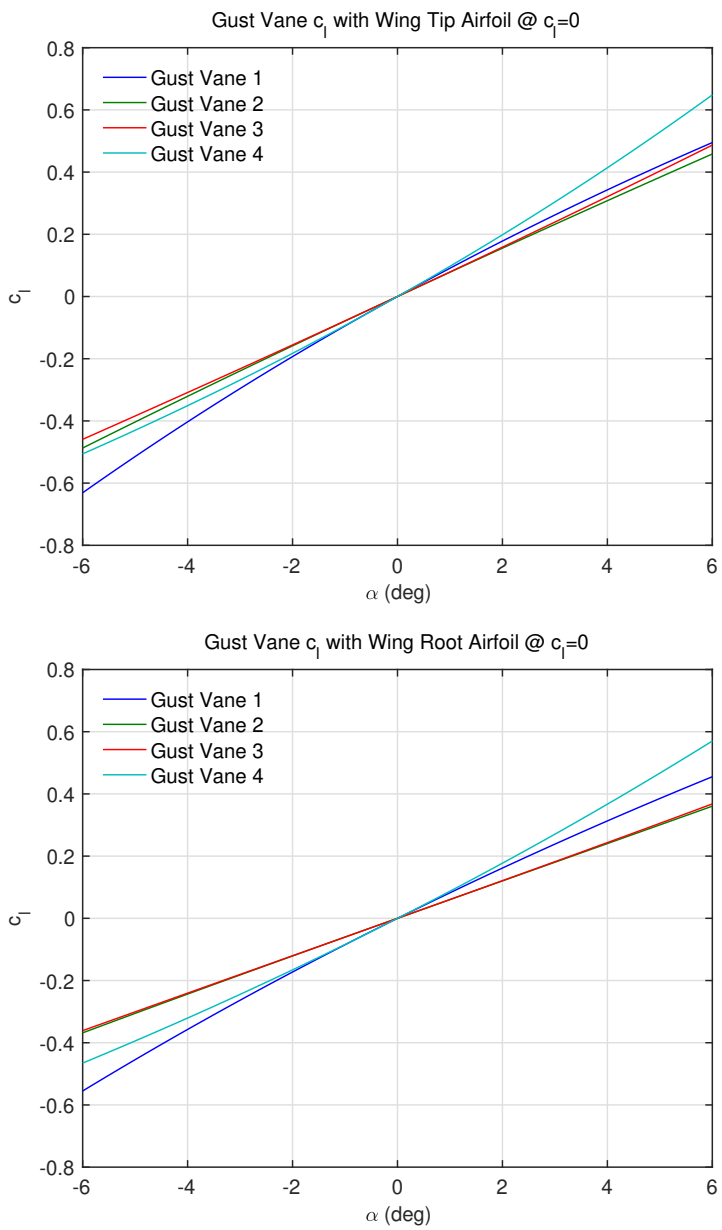

Figure 26. Vortex Model of Gust Generator-Wing System

\begin{tabular}{|c|c|c|c|c|}
\hline$c_{L_{\alpha}}$ & Average Gust Vane & Wing Root & Wing MAC & Wing Tip \\
\hline Gust Vanes + Wing Root & 4.1698 & 5.5913 & & \\
\hline Gust Vanes + Wing MAC & 4.6427 & & 5.9716 & \\
\hline Gust Vanes + Wing Tip & 4.9644 & & & 6.2364 \\
\hline Gust Vanes Alone & 5.0216 & $2 \pi$ & $2 \pi$ & $2 \pi$ \\
\hline
\end{tabular}

Table 5. Lift Curve Slope $c_{L_{\alpha}}$ for Gust Vane - Wing Configurations 


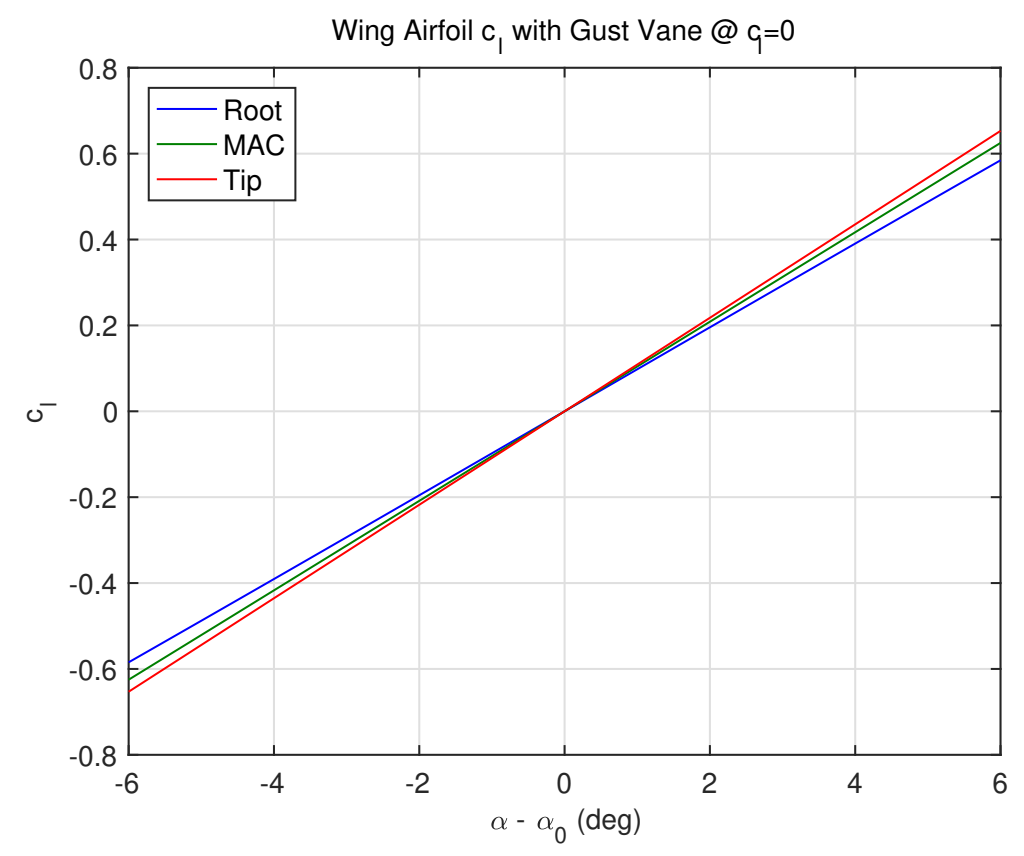

Figure 27. Lift Curves of Wing Airfoils + Gust Vanes @ $c_{L}=0$

Figure 28 shows the variation of the ratios of the lift curve slopes to the theoretical lift curve slope value of $2 \pi$, denoted as $p$, for the three CRM wing airfoils with the angle of attack of the gust vanes. Thus, $p$ represents an aerodynamic interference factor due to the gust vane interaction with the wing airfoil. This aerodynamic interference is the greatest when the gust vanes are at the most negative angle of attack $\alpha_{v}=-\theta_{v}$ and the wing airfoil is the closest to the gust vanes. Thus, the three-dimensional lift curve slope $c_{L_{\alpha}}$ can be corrected in a stripwise fashion by the parameter $p$.

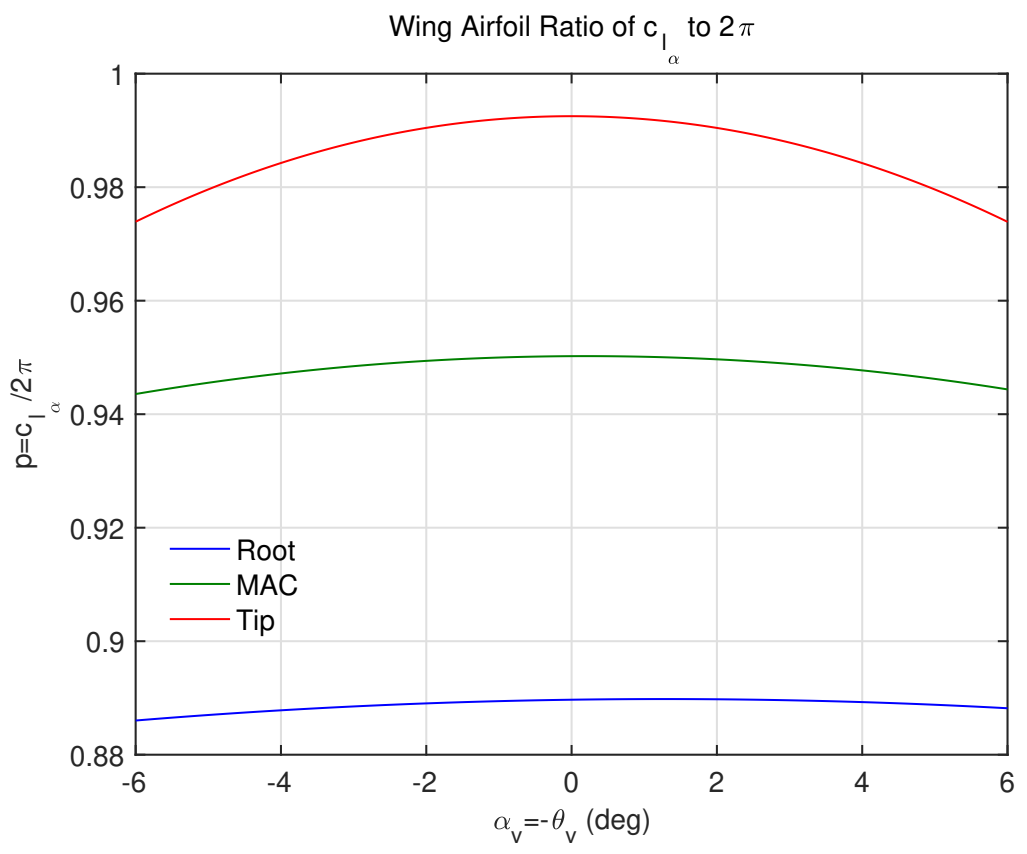

Figure 28. Variation of Lift Curve Slopes of Wing Airfoils 


\section{Unsteady Lift Due to Gust}

When the gust vertical velocity begins to strike the leading edge of the wing section, an unsteady lift is developed and can be computed by the Duhamel's integral as

$$
\bar{l}_{g}=q_{\infty} c c_{L_{\alpha}}\left[\alpha_{g}(0) \psi(\tau)+\int_{0}^{\tau} \frac{d \alpha_{g}(\sigma)}{d \sigma} \psi(\tau-\sigma) d \sigma\right]
$$

where $\tau=\frac{2 V_{\infty} t}{c}$ is the distance in semi-chords traveled by the airfoil and $\psi(\tau)$ is the Küssner's function. ${ }^{22}$

The unsteady lift can then be expressed as

$$
\bar{l}_{g}=q_{\infty} c c_{L_{\alpha}} r_{g} \alpha_{g}\left(t-t_{g}\right)
$$

where $r_{g}$ is the amplitude ratio and $t_{g}$ is the time lag due to the Küssner's function which can be estimated by evaluating the Duhamel's integral.

The resulting steady state unsteady lift due to gust can be expressed as

$$
\bar{l}_{g}=q_{\infty} c c_{L_{\alpha}} r_{g} r\left[-\cos \omega\left(t_{d}+t_{g}\right) \theta_{v}+\sin \omega\left(t_{d}+t_{g}\right) \frac{\dot{\theta}_{v}}{\omega}\right]
$$

For a sinusoidal gust with $\alpha_{g}=\left|\alpha_{g}\right| e^{i \omega t}$, the unsteady lift can also be evaluated as

$$
\bar{l}_{g}=q_{\infty} c c_{L_{\alpha}} C_{s g}(k) \alpha_{g}
$$

where

$$
C_{s g}(k)=C(k)\left[J_{0}(k)-i J_{1}(k)\right]+i J_{1}(k)=F_{s g}(k)+i G_{s g}(k)
$$

and $J_{0}$ and $J_{1}$ are the Bessel's functions. ${ }^{22}$

The steady state unsteady lift then becomes

$$
\begin{aligned}
\bar{l}_{g}=q_{\infty} c c_{L_{\alpha}}\left[F_{s g}(k)\right. & \left.\alpha_{g}+G_{s g}(k) \frac{\dot{\alpha}_{g}}{\omega}\right] \\
= & q_{\infty} c c_{L_{\alpha}} r\left\{-\left[F_{s g}(k) \cos \omega t_{d}+G_{s g}(k) \sin \omega t_{d}\right] \theta_{v}+\left[F_{s g}(k) \sin \omega t_{d}-G_{s g}(k) \cos \omega t_{d}\right] \frac{\dot{\theta}_{v}}{\omega}\right\}
\end{aligned}
$$

Equating the unsteady lift expressions in Eqs. (31) and (34) yields

$$
\begin{aligned}
& r_{g} \cos \omega\left(t_{d}+t_{g}\right)=F_{s g}(k) \cos \omega t_{d}+G_{s g}(k) \sin \omega t_{d} \\
& r_{g} \sin \omega\left(t_{d}+t_{g}\right)=F_{s g}(k) \sin \omega t_{d}-G_{s g}(k) \cos \omega t_{d}
\end{aligned}
$$

from which $r_{g}$ and $t_{g}$ are obtained as

$$
\begin{gathered}
r_{g}=\sqrt{F_{s g}^{2}(k)+G_{s g}^{2}(k)} \\
t_{g}=\frac{1}{\omega} \tan ^{-1} \frac{F_{s g}(k) \sin \omega t_{d}-G_{s g}(k) \cos \omega t_{d}}{F_{s g}(k) \cos \omega t_{d}+G_{s g}(k) \sin \omega t_{d}}-t_{d}
\end{gathered}
$$

The unsteady pitching moment as a result of the unsteady lift can be estimated as

$$
\bar{m}_{g}=e \bar{l}_{g}
$$

Expressing the unsteady lift and pitching moment due to gust as

$$
\begin{gathered}
l_{g}=\bar{l}_{g} \cos \Lambda=l_{\theta_{v}} \theta_{v}+l_{\dot{\theta}_{v}} \dot{\theta}_{v} \\
m_{g}=\bar{m}_{g} \cos \Lambda \sin \Lambda=m_{\theta_{v}}+m_{\dot{\theta}_{v}}
\end{gathered}
$$

where

$$
\begin{aligned}
l_{\theta_{v}} & =-q_{\infty} c c_{L_{\alpha}} r_{g} r \cos \omega\left(t_{d}+t_{g}\right) \cos \Lambda \\
l_{\dot{\theta}_{v}} & =q_{\infty} c c_{L_{\alpha}} \frac{r_{g} r}{\omega} \sin \omega\left(t_{d}+t_{g}\right) \cos \Lambda
\end{aligned}
$$




$$
\begin{aligned}
m_{\theta_{v}} & =-q_{\infty} \operatorname{cec}_{L_{\alpha}} r_{g} r \cos \omega\left(t_{d}+t_{g}\right) \cos \Lambda \sin \Lambda \\
m_{\dot{\theta}_{v}} & =q_{\infty} c e c_{L_{\alpha}} \frac{r_{g} r}{\omega} \sin \omega\left(t_{d}+t_{g}\right) \cos \Lambda \sin \Lambda
\end{aligned}
$$

The generalized aerodynamic force due to gust is evaluated as

$$
F_{g}=F_{\theta_{v}} \theta_{v}+F_{\dot{\theta}_{v}} \dot{\theta}_{v}+\Delta F_{g}
$$

where

$$
\begin{aligned}
& F_{\theta_{v}}=\int_{0}^{L}\left(\Phi^{\top} l_{\theta_{v}}-\Phi^{\prime} m_{\theta_{v}}\right) d x \\
& F_{\dot{\theta}_{v}}=\int_{0}^{L}\left(\Phi^{\top} l_{\dot{\theta}_{v}}-\Phi^{\prime} m_{\dot{\theta}_{v}}\right) d x
\end{aligned}
$$

The integrated unsteady lift and pitching moment about the pitch axis are obtained as

$$
\begin{gathered}
L_{g}=L_{\theta_{v}} \theta_{v}+L_{\dot{\theta}_{v}} \dot{\theta}_{v} \\
M_{g}=M_{\theta_{v}} \theta_{v}+M_{\dot{\theta}_{v}} \dot{\theta}_{v}
\end{gathered}
$$

where

$$
\begin{aligned}
L_{\theta_{v}} & =\int_{0}^{L} l_{\theta_{v}} d x \\
L_{\dot{\theta}_{v}} & =\int_{0}^{L} l_{\dot{\theta}_{v}} d x \\
M_{\theta_{v}} & =\int_{0}^{L} e_{p} l_{\theta_{v}} d x \\
M_{\dot{\theta}_{v}} & =\int_{0}^{L} e_{p} l_{\dot{\theta}_{v}} d x
\end{aligned}
$$

To assess how well the unsteady lift model is able to predict the gust load on the wing, the unsteady lift computed by the model is compared to the CFD simulations results. Figures 29 - 31 show the unsteady lift at the three CRM wing stations for a gust vane sinusoidal input at $2 \mathrm{~Hz}$ frequency and $5^{\circ}$ amplitude for a dynamic pressure of 10 psf. The unsteady lift model produces good agreement with the CFD result at the wing root, but has poor agreement at the wing tip. This is likely due to the sharp drop-off in the gust angle of attack at the wing tip leading edge in the model, corresponding to $\frac{d}{c_{g}}=5.27$, as seen in Fig. 23. Nonetheless, the in absolute sense, the disagreement in the unsteady lift at the wing tip only amounts to less than $1 \mathrm{lb} / \mathrm{ft}$ which is about the same as the differences in the unsteady lift at the wing root and MAC stations. In future work, we plan to further improve the unsteady lift model. 


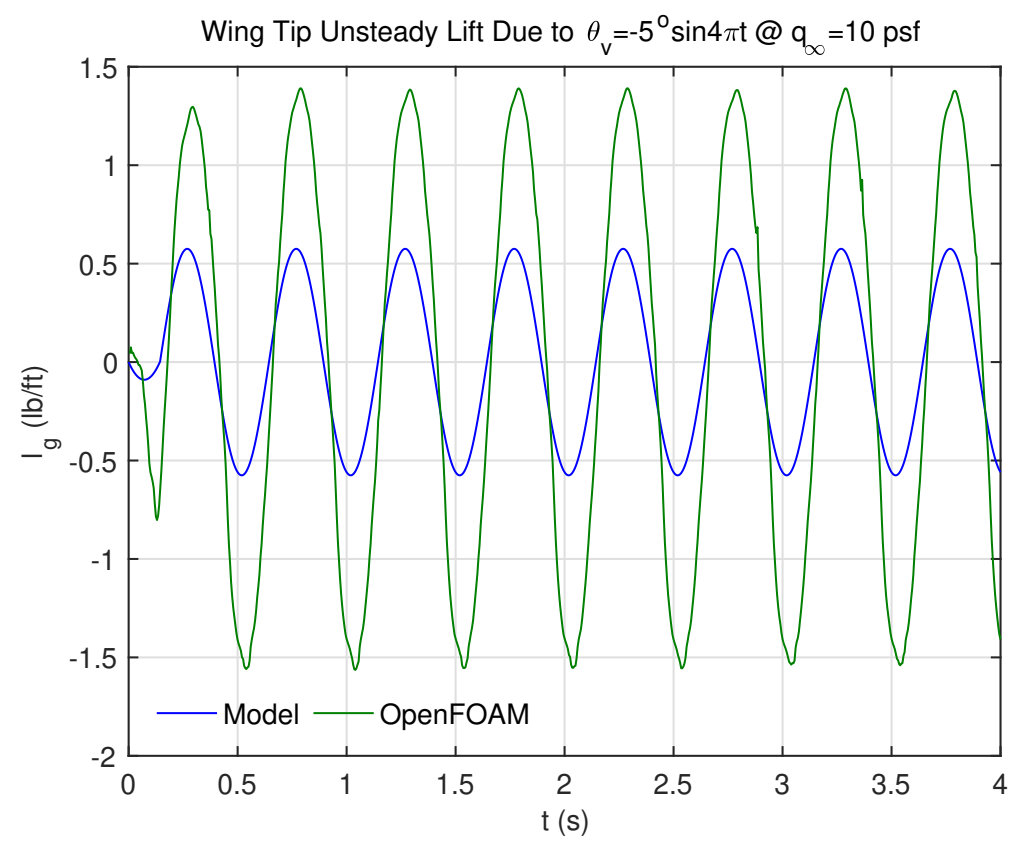

Figure 29. Unsteady Lift at CRM Wing Tip

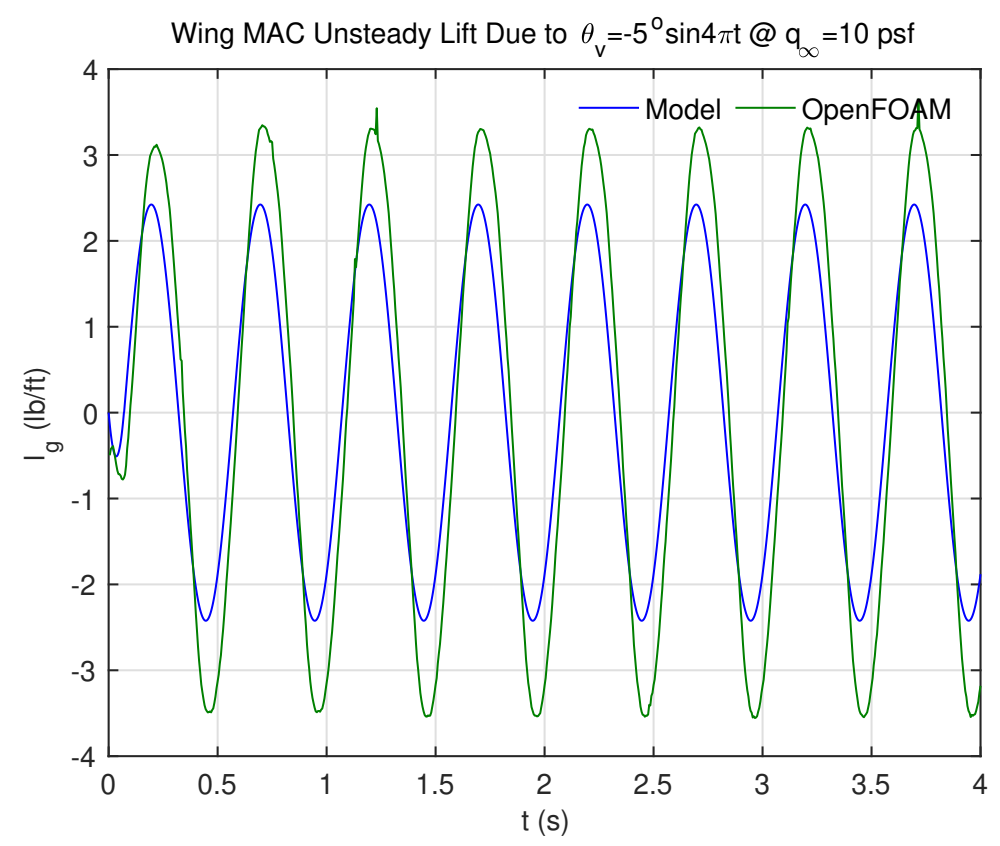

Figure 30. Unsteady Lift at CRM Wing CRM 


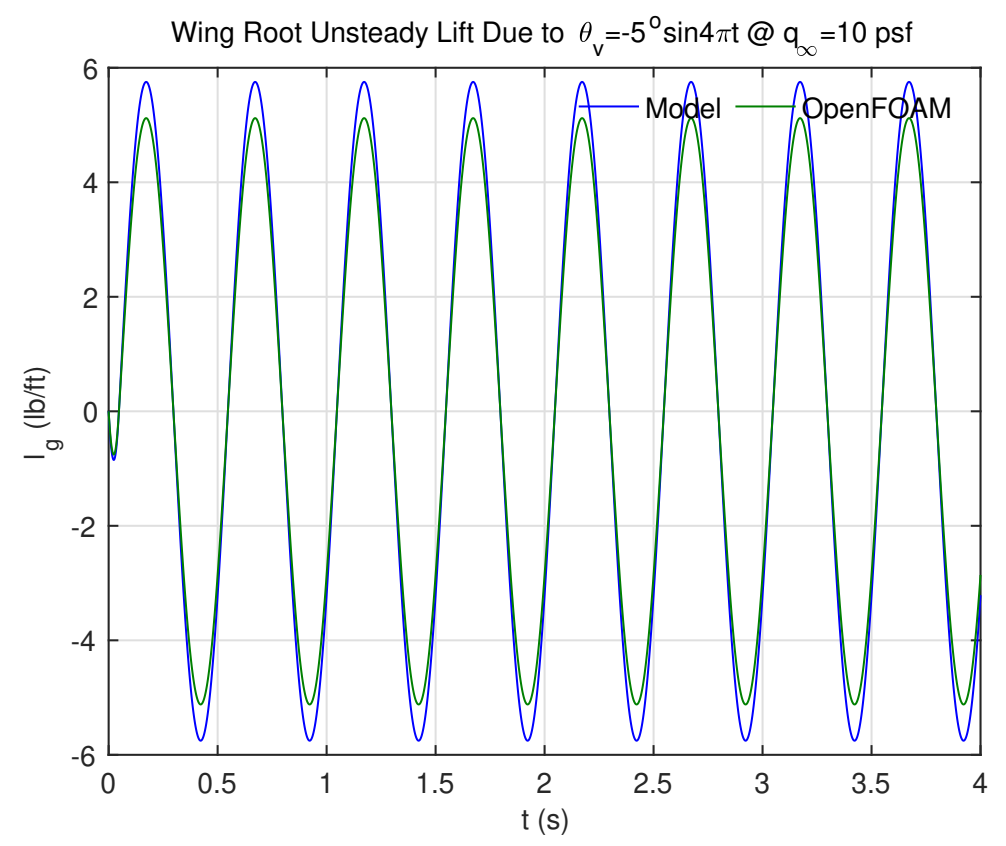

Figure 31. Unsteady Lift at CRM Wing Root

Figure 32 shows the total integrated unsteady lift which has an amplitude of $11.08 \mathrm{lbs}$. Experimental data for unsteady lift are available from the wind tunnel test. Figures 33 and 34are the plots of the lift force measurement from the side wall balance and the gust vane pitch angle response to a sinusoidal input of $5^{\circ}$ amplitude at a $2 \mathrm{~Hz}$ frequency taken on August 135, 2019 from Run 64. The measured unsteady lift is $13.26 \mathrm{lbs}$. This unsteady lift also includes the wing dynamic response, so a direct comparison is not possible. In Section IV, the computed unsteady lift response of the wing is plotted in Fig. 36. For the open-loop response without active controls, the computed unsteady lift response is $13.54 \mathrm{lbs}$. This indicates that the unsteady lift model produces an unsteady lift response that is in excellent agreement with the measured unsteady lift response.

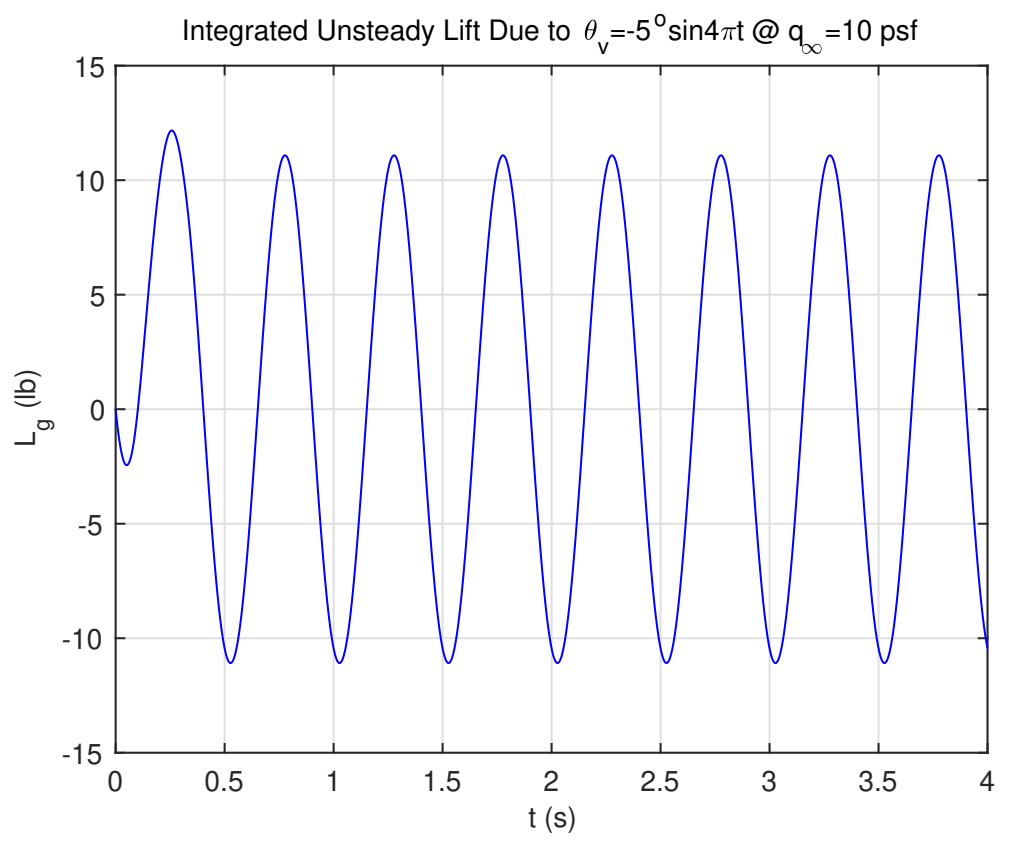

Figure 32. Total Integrated Unsteady Lift 


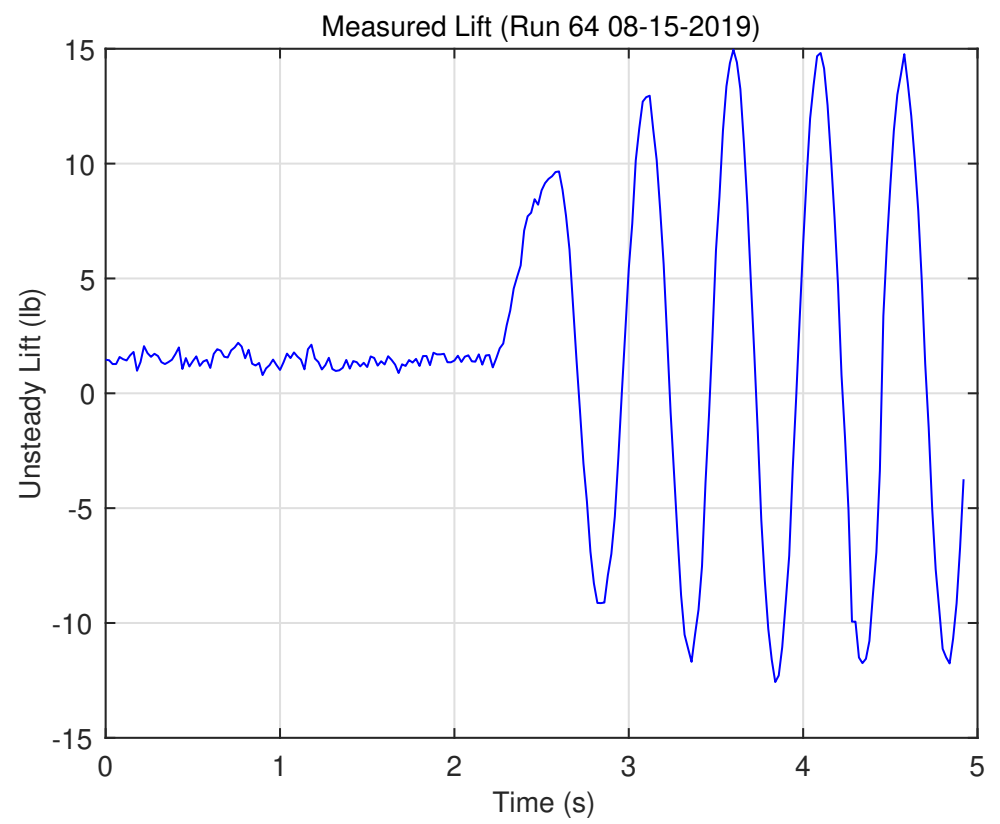

Figure 33. Measured Unsteady Lift Response

The aerodynamic lag of the unsteady lift response can be inferred from Fig. 34 which shows the zero-mean unsteady lift in relation to the gust vane pitch angle. When the gust vane pitch angle is negative, a positive lift is generated. An aerodynamic lag of $0.12 \mathrm{sec}$ is observed between the unsteady lift response and the gust vane pitch angle. We note that the sampling frequency of the data is $50 \mathrm{~Hz}$ at a time step of $0.02 \mathrm{sec}$. The integrated lift in Fig. 32 shows a $0.13 \mathrm{sec}$ lag. In Section IV, a $0.14 \mathrm{sec}$ lag is observed from Fig. 36 for the open-loop response. Thus, the aerodynamic lag is accurately captured by the unsteady lift model.

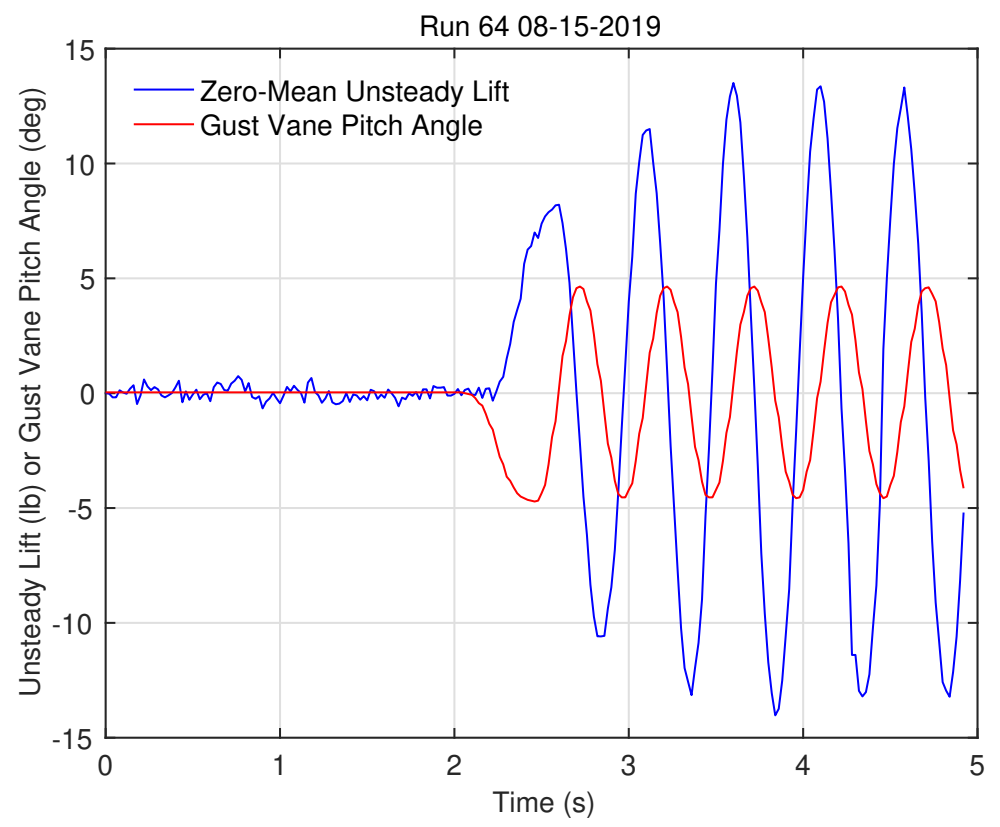

Figure 34. Measured Gust Vane Pitch Angle 


\section{ASE State-Space Model}

We consider two different formulations of ASE state-space models: 1) frequency-dependent formulation and 2) frequency-independent formulation. The ASE state-space model is expressed in the most general form as

$$
\begin{aligned}
& \dot{x}=A x+B u+E w \\
& y=C x+D u+F w
\end{aligned}
$$

where $x$ is the state vector to be re-constructed from the output vector $y$ which represents the accelerometer signals, $u$ is the command vector of the flap deflections, and $w=\left[\begin{array}{cc}\theta_{v} & \dot{\theta}_{v}\end{array}\right]^{\top}$ is a gust input vector. We note that the statespace model is about the equilibrium value of $\bar{q}=\bar{K}^{-1}\left(F_{0}+F_{\alpha} \alpha+F_{\delta} \bar{\delta}\right)$, where $\bar{q}$ is the steady-state generalized displacement.

In addition, an unsteady lift model is included

$$
L=\bar{L}+\bar{L}_{x} x+\bar{L}_{u} u+\bar{L}_{w} w
$$

where $\bar{L}$ is the steady-state lift. This unsteady lift is measured directly by the side wall balance. It should be noted that originally strain gauges were installed on the wing spar for the purpose of measuring the wing bending moment to be used in the gust load alleviation control laws. These strain gauges were determined to be non-functional. As a result, the unsteady lift measurement from the side wall balance is to be used as a replacement for the wing root bending moment.

\section{Actuator Model}

The actuator dynamics are modeled as

$$
\ddot{\delta}+2 \zeta_{a} \omega_{a} \dot{\delta}+\omega_{a}^{2} \delta=d u
$$

where $\omega_{a}$ is the frequency of the actuator which is set at $9 \mathrm{~Hz}, \zeta_{a}$ is the viscous damping ratio, and $d$ is a conversion constant.

The command vector $u$ is then used to compute the actual command vector $\delta_{c}$ to the flap deflections via a servo PID (proportional-integral-derivative) control

$$
u=-k_{p}\left(\delta-\delta_{c}\right)-k_{i} \int\left(\delta-\delta_{c}\right) d \tau-k_{d}\left(\dot{\delta}-\dot{\delta}_{c}\right)
$$

where $\left(k_{p}, k_{i}, k_{d}\right)$ are the proportional-integral-derivative control gains.

\section{State-Space Model}

We present the most general form of the ASE state-space model using the frequency-independent formulation. The other forms of the ASE state-space models are provided in Appendix B.

The equation of motion is

$$
\begin{aligned}
\left(M+M_{a}\right) \ddot{q}+\left[C+C_{a}+C(k) C_{a}^{*}\right] \dot{q}+[K+C(k) & \left.K_{a}^{*}\right] q=F_{0}+F_{\alpha} \alpha \\
& +F_{\delta} \bar{\delta}+C(k) F_{\delta}^{*} \delta+F_{\dot{\delta}} \dot{\delta}+C(k) F_{\dot{\delta}}^{*} \dot{\delta}+F_{\ddot{\delta}} \ddot{\delta}+F_{\theta_{v}} \theta_{v}+F_{\dot{\theta}_{v}} \dot{\theta}_{v}
\end{aligned}
$$

where $M$ and $K$ are the NASTRAN generalized mass and stiffness matrices scaled appropriately, and $C=2 M \zeta \omega$ is the damping matrix where $\zeta$ is the viscous damping ratio selected to be $2.5 \%$ and $\omega$ is the diagonal matrix of the natural frequencies.

The Theodorsen's function is approximated by the R .T. Jones approximation ${ }^{23}$

$$
C(k) \approx \hat{C}(\bar{s})=\frac{0.5 \bar{s}^{2}+a_{1} \bar{s}+a_{2}}{\bar{s}^{2}+a_{3} \bar{s}+a_{2}}
$$

where $a_{1}=0.2814, a_{2}=0.01463, a_{3}=0.3492$, and $\bar{s}=\frac{s \bar{c}}{2 V_{\infty}}$ is a dimensionless Laplace transform variable. Then,

$$
C(k) q \approx \hat{C}(\bar{s}) q=\frac{0.5 \bar{s}^{2}+a_{1} \bar{s}+a_{2}}{\bar{s}^{2}+a_{3} \bar{s}+a_{2}} q=0.5 q+\frac{a_{4} \bar{s}+0.5 a_{2}}{\bar{s}^{2}+a_{3} \bar{s}+a_{2}} q
$$




$$
C(k) \dot{q} \approx \hat{C}(\bar{s}) s q=\frac{0.5 \bar{s}^{3}+a_{1} \bar{s}^{2}+a_{2} \bar{s}}{\bar{s}^{2}+a_{3} \bar{s}+a_{2}}\left(\frac{2 V_{\infty}}{\bar{c}}\right) q=0.5 \dot{q}+a_{4}\left(\frac{2 V_{\infty}}{\bar{c}}\right) q+\frac{a_{5} \bar{s}+a_{6}}{\bar{s}^{2}+a_{3} \bar{s}+a_{2}}\left(\frac{2 V_{\infty}}{\bar{c}}\right) q
$$

where $a_{4}=a_{1}-0.5 a_{3}=0.1068, a_{5}=0.5 a_{2}-a_{3} a_{4}=-0.03$, and $a_{6}=-a_{2} a_{4}=-0.0016$.

The following aerodynamic lag states are introduced

$$
\begin{aligned}
& y_{q}=\frac{a_{4} \bar{s}+0.5 a_{2}}{\bar{s}^{2}+a_{3} \bar{s}+a_{2}} q \\
& z_{q}=\frac{a_{5} \bar{s}+a_{6}}{\bar{s}^{2}+a_{3} \bar{s}+a_{2}} q
\end{aligned}
$$

These equations are expressed in the time domain as

$$
\begin{gathered}
\ddot{y}_{q}+a_{3}\left(\frac{2 V_{\infty}}{\bar{c}}\right) \dot{y}_{q}+a_{2}\left(\frac{2 V_{\infty}}{\bar{c}}\right)^{2} y_{q}=a_{4}\left(\frac{2 V_{\infty}}{\bar{c}}\right) \dot{q}+0.5 a_{2}\left(\frac{2 V_{\infty}}{\bar{c}}\right)^{2} q \\
\ddot{z}_{q}+a_{3}\left(\frac{2 V_{\infty}}{\bar{c}}\right) \dot{z}_{q}+a_{2}\left(\frac{2 V_{\infty}}{\bar{c}}\right)^{2} z_{q}=a_{5}\left(\frac{2 V_{\infty}}{\bar{c}}\right) \dot{q}+a_{6}\left(\frac{2 V_{\infty}}{\bar{c}}\right)^{2} q
\end{gathered}
$$

Then, the equation of motion is expressed as

$$
\begin{aligned}
\left(M+M_{a}\right) \ddot{q}+\left(C+C_{a}+0.5 C_{a}^{*}\right) \dot{q}+\left[K+0.5 K_{a}^{*}+a_{4}\left(\frac{2 V_{\infty}}{\bar{c}}\right) C_{a}^{*}\right] q & \\
& +K_{a}^{*} y_{q}+\left(\frac{2 V_{\infty}}{\bar{c}}\right) C_{a}^{*} z_{q}=F_{0}+F_{\alpha} \alpha+F_{c}+F_{g}
\end{aligned}
$$

Using the R. T. Jones approximation of the Theodorsen's function, the generalized aerodynamic force due to the flap motion is obtained as

$$
\begin{gathered}
F_{c}=F_{\delta} \bar{\delta}+\left[F_{\delta}+0.5 F_{\delta}^{*}+a_{4}\left(\frac{2 V_{\infty}}{\bar{c}}\right) F_{\dot{\delta}}^{*}\right] \delta+\left(F_{\dot{\delta}}+0.5 F_{\dot{\delta}}^{*}\right) \dot{\delta}+F_{\ddot{\delta}} \ddot{\delta}+F_{\delta}^{*} y_{\delta}+\left(\frac{2 V_{\infty}}{\bar{c}}\right) F_{\dot{\delta}}^{*} z_{\delta} \\
\ddot{y}_{\delta_{i}}+a_{3}\left(\frac{2 V_{\infty}}{\bar{c}}\right) \dot{y}_{\delta_{i}}+a_{2}\left(\frac{2 V_{\infty}}{\bar{c}}\right)^{2} y_{\delta_{i}}=a_{4}\left(\frac{2 V_{\infty}}{\bar{c}}\right) \dot{\delta}_{i}+0.5 a_{2}\left(\frac{2 V_{\infty}}{\bar{c}}\right)^{2} \delta_{i} \\
\ddot{z}_{\delta_{i}}+a_{3}\left(\frac{2 V_{\infty}}{\bar{c}}\right) \dot{z}_{\delta_{i}}+a_{2}\left(\frac{2 V_{\infty}}{\bar{c}}\right)^{2} z_{\delta_{i}}=a_{5}\left(\frac{2 V_{\infty}}{\bar{c}}\right) \dot{\delta}_{i}+a_{6}\left(\frac{2 V_{\infty}}{\bar{c}}\right)^{2} \delta_{i}
\end{gathered}
$$

Let $x=\left[\begin{array}{cccccccccccc}q & \dot{q} & y_{q} & \dot{y}_{q} & z_{q} & \dot{z}_{q} & \delta & \dot{\delta} & y_{\delta} & \dot{y}_{\delta} & z_{\delta} & \dot{z}_{\delta}\end{array}\right]^{\top}$. Then, the state-space model is described by

$$
\begin{aligned}
& A=\left[\begin{array}{c|c}
A_{11} & A_{12} \\
\hline 0 & A_{22}
\end{array}\right] \\
& B=\left[\begin{array}{llllllllllll}
0 & \bar{M}^{-1} F_{\ddot{\delta}} d & 0 & 0 & 0 & 0 & 0 & d & 0 & 0 & 0 & 0
\end{array}\right]^{\top} \\
& E=\left[\begin{array}{ccccccccccccc}
0 & \bar{M}^{-1} F_{\theta_{v}} & 0 & 0 & 0 & 0 & 0 & 0 & 0 & 0 & 0 & 0 \\
0 & \bar{M}^{-1} F_{\dot{\theta}_{v}} & 0 & 0 & 0 & 0 & 0 & 0 & 0 & 0 & 0 & 0
\end{array}\right]^{\top}
\end{aligned}
$$

where

$$
A_{11}=\left[\begin{array}{cccccc}
0 & I & 0 & 0 & 0 & 0 \\
-\bar{M}^{-1} \bar{K} & -\bar{M}^{-1} \bar{C} & -\bar{M}^{-1} K_{a}^{*} & 0 & -\bar{M}^{-1} C_{a}^{*}\left(\frac{2 V_{\infty}}{\bar{c}}\right) & 0 \\
0 & 0 & 0 & I & 0 & 0 \\
0.5 a_{2}\left(\frac{2 V_{\infty}}{\bar{c}}\right)^{2} & a_{4}\left(\frac{2 V_{\infty}}{\bar{c}}\right) & -a_{2}\left(\frac{2 V_{\infty}}{\bar{c}}\right)^{2} & -a_{3}\left(\frac{2 V_{\infty}}{\bar{c}}\right) & 0 & 0 \\
0 & 0 & 0 & 0 & 0 & I \\
a_{6}\left(\frac{2 V_{\infty}}{\bar{c}}\right)^{2} & a_{5}\left(\frac{2 V_{\infty}}{\bar{c}}\right) & 0 & 0 & -a_{2}\left(\frac{2 V_{\infty}}{\bar{c}}\right)^{2} & -a_{3}\left(\frac{2 V_{\infty}}{\bar{c}}\right)
\end{array}\right]
$$




$$
\begin{aligned}
& A_{12}=\left[\begin{array}{cccccc}
0 & 0 & 0 & 0 & 0 & 0 \\
\bar{M}^{-1} \bar{F}_{\delta} & \bar{M}^{-1} \bar{F}_{\dot{\delta}} & \bar{M}^{-1} F_{\delta}^{*} & 0 & \bar{M}^{-1} F_{\dot{\delta}}^{*}\left(\frac{2 V_{\infty}}{\bar{c}}\right) & 0 \\
0 & 0 & 0 & 0 & 0 & 0 \\
0 & 0 & 0 & 0 & 0 & 0 \\
0 & 0 & 0 & 0 & 0 & 0 \\
0 & 0 & 0 & 0 & 0 & 0
\end{array}\right] \\
& A_{22}=\left[\begin{array}{cccccc}
0 & I & 0 & 0 & 0 & 0 \\
-\omega_{a}^{2} & -2 \zeta_{a} \omega_{a} & 0 & 0 & 0 & 0 \\
0 & 0 & 0 & I & 0 & 0 \\
0.5 a_{2}\left(\frac{2 V_{\infty}}{\bar{c}}\right)^{2} & a_{4}\left(\frac{2 V_{\infty}}{\bar{c}}\right) & -a_{2}\left(\frac{2 V_{\infty}}{\bar{c}}\right)^{2} & -a_{3}\left(\frac{2 V_{\infty}}{\bar{c}}\right) & 0 & 0 \\
0 & 0 & 0 & 0 & 0 & I \\
a_{6}\left(\frac{2 V_{\infty}}{\bar{c}}\right)^{2} & a_{5}\left(\frac{2 V_{\infty}}{\bar{c}}\right) & 0 & 0 & -a_{2}\left(\frac{2 V_{\infty}}{\bar{c}}\right)^{2} & -a_{3}\left(\frac{2 V_{\infty}}{\bar{c}}\right)
\end{array}\right] \\
& \bar{M}=M+M_{a} \\
& \bar{C}=C+C_{a}+0.5 C_{a}^{*} \\
& \bar{K}=K+0.5 K_{a}^{*}+a_{4}\left(\frac{2 V_{\infty}}{\bar{c}}\right) C_{a}^{*} \\
& \bar{F}_{\delta}=F_{\delta}+0.5 F_{\delta}^{*}+a_{4}\left(\frac{2 V_{\infty}}{\bar{c}}\right) F_{\dot{\delta}}^{*}-F_{\ddot{\delta}} \omega_{a}^{2} \\
& \bar{F}_{\dot{\delta}}=F_{\dot{\delta}}+0.5 F_{\dot{\delta}}^{*}-F_{\ddot{\delta}} 2 \zeta_{a} \omega_{a}
\end{aligned}
$$

\section{Output Model}

The output vector $y$ represents the acceleration measurements from the accelerometers. The acceleration at a specified location $x_{a}$ on the elastic axis, offset by an distance $e_{a}$ where $e_{a}>0$ if the acceleration location is forward of the elastic axis, is modeled as

$$
\ddot{z}_{a}=\left[\Phi\left(x_{a}\right)+e_{a} \Psi\left(x_{a}\right)\right] \ddot{q}=\left[\Phi\left(x_{a}\right)+e_{a} \Psi\left(x_{a}\right)\right] I_{\ddot{q}}(A x+B u+E w)
$$

where $I_{\ddot{q}}=\left[\begin{array}{llllllllllll}0 & I & 0 & 0 & 0 & 0 & 0 & 0 & 0 & 0 & 0 & 0\end{array}\right]$ such that $\ddot{q}=I_{\ddot{q}} \dot{x}$.

Then,

$$
y=\ddot{z}_{a}=C x+D u+F w
$$

where

$$
\begin{aligned}
& C=\left[\Phi\left(x_{a}\right)+e_{a} \Psi\left(x_{a}\right)\right] I_{\dot{q}} A \\
& D=\left[\Phi\left(x_{a}\right)+e_{a} \Psi\left(x_{a}\right)\right] I_{\dot{q}} B \\
& F=\left[\Phi\left(x_{a}\right)+e_{a} \Psi\left(x_{a}\right)\right] I_{\dot{q}} E
\end{aligned}
$$

\section{Unsteady Lift Model}

The unsteady lift is given by

$$
\begin{aligned}
L=L_{a}+L_{c}+L_{g}+L_{i}+L_{d}=q_{\infty} S & \left(C_{L_{0}}+C_{L_{\alpha}} \alpha\right)+C(k) L_{q}^{*} q+C(k) L_{\dot{q}}^{*} \dot{q}+L_{\dot{q}} \dot{q} \\
& +L_{\ddot{q}} \ddot{q}+q_{\infty} S C_{L_{\delta}} \bar{\delta}+C(k) L_{\delta}^{*} \delta+C(k) L_{\dot{\delta}}^{*} \dot{\delta}+L_{\dot{\delta}} \dot{\delta}+L_{\ddot{\delta}} \ddot{\delta}+L_{\theta_{v}} \theta_{v}+L_{\dot{\theta}_{v}} \dot{\theta}_{v}
\end{aligned}
$$

where $L_{i}=\Delta L_{\ddot{q}} \ddot{q}$ and $L_{d}=\Delta L_{\dot{q}} \dot{q}$ are due to the inertial and damping forces. Using the approximation of the Theodorsen's function, the unsteady lift is given by

$$
\begin{aligned}
L=q_{\infty} & S\left(C_{L_{0}}+C_{L_{\alpha}} \alpha+C_{L_{\delta}} \bar{\delta}\right)+\left[0.5 L_{q}^{*}+a_{4}\left(\frac{2 V_{\infty}}{\bar{c}}\right) L_{\dot{q}}^{*}\right] q+\left(L_{\dot{q}}+0.5 L_{\dot{q}}^{*}+\Delta L_{\dot{q}}\right) \dot{q}+\left(L_{\ddot{q}}+\Delta L_{\ddot{q}}\right) \ddot{q}+L_{q}^{*} y_{q} \\
& +\left(\frac{2 V_{\infty}}{\bar{c}}\right) L_{\dot{q}}^{*} z_{q}+\left[0.5 L_{\delta}^{*}+a_{4}\left(\frac{2 V_{\infty}}{\bar{c}}\right) L_{\dot{\delta}}^{*}\right] \delta+\left(L_{\dot{\delta}}+0.5 L_{\dot{\delta}}^{*}\right) \dot{\delta}+L_{\ddot{\delta}} \ddot{\delta}+L_{\delta}^{*} y_{\delta}+\left(\frac{2 V_{\infty}}{\bar{c}}\right) L_{\dot{\delta}}^{*} z_{\delta}+L_{w} w
\end{aligned}
$$


The unsteady lift is then expressed as

$$
L=\bar{L}+\bar{L}_{x} x+\bar{L}_{u} u+\bar{L}_{w} w
$$

where

$$
\begin{aligned}
& \bar{L}_{x}=L_{x}+\bar{L}_{\ddot{q}} I_{\ddot{q}} A \\
& \bar{L}_{u}=L_{u}+\bar{L}_{\ddot{q}} I_{\ddot{q}} B \\
& \bar{L}_{w}=L_{w}+\bar{L}_{\ddot{q}} I_{\ddot{q}} E \\
& L_{x}=\left[\begin{array}{llllllllllll}
\bar{L}_{q} & \bar{L}_{\dot{q}} & L_{q}^{*} & 0 & \left(\frac{2 V_{\infty}}{\bar{c}}\right) L_{\dot{q}}^{*} & 0 & \bar{L}_{\delta} & \bar{L}_{\dot{\delta}} & L_{\delta}^{*} & 0 & \left(\frac{2 V_{\infty}}{\bar{c}}\right) L_{\dot{\delta}}^{*} & 0
\end{array}\right] \\
& L_{u}=L_{\ddot{\delta}} d \\
& L_{w}=\left[\begin{array}{ll}
L_{\theta_{v}} & L_{\dot{\theta}_{v}}
\end{array}\right] \\
& \bar{L}_{q}=0.5 L_{q}^{*}+a_{4}\left(\frac{2 V_{\infty}}{\bar{c}}\right) L_{\dot{q}}^{*} \\
& \bar{L}_{\dot{q}}=L_{\dot{q}}+0.5 L_{\dot{q}}^{*}+\Delta L_{\dot{q}} \\
& \bar{L}_{\ddot{q}}=L_{\ddot{q}}+\Delta L_{\ddot{q}} \\
& \bar{L}_{\delta}=0.5 L_{\delta}^{*}+a_{4}\left(\frac{2 V_{\infty}}{\bar{c}}\right) L_{\dot{\delta}}^{*}-L_{\ddot{\delta}} \omega_{a}^{2} \\
& \bar{L}_{\dot{\delta}}=L_{\dot{\delta}}+0.5 L_{\dot{\delta}}^{*}-L_{\ddot{\delta}} 2 \zeta_{a} \omega_{a}
\end{aligned}
$$

\section{Multi-Objective Control}

A multi-objective control law is designed for gust load alleviation and drag optimization. ${ }^{17-20}$ The multi-objective control is unique in a way that it enables a control synthesis that accounts for multiple control objectives that co-exist in a control law. This is enabled by the existence of distributed control surfaces such as the VCCTEF. Two important objectives in flight control of flexible transport aircraft are fuel efficiency and ride quality which is a measure of passenger comfort. So, drag and load becomes two important metrics that need to be accounted for in a flight control system. The multi-objective flight control can allow a trade-off among the different competing control objectives through a Pareto optimal analysis. ${ }^{19}$

Consider the following multi-objective cost function:

$$
J=\frac{1}{2} \lim _{t_{f} \rightarrow \infty} \int_{0}^{t_{f}}\left[x^{\top} Q x+u^{\top} R u+q_{D}(\Delta \bar{D}+\Delta D)+q_{L}(\Delta \bar{L}+\Delta L)^{2}\right] d t
$$

where $\Delta \bar{L}$ and $\Delta \bar{D}$ are the incremental steady-state lift and drag, and $\Delta L$ and $\Delta D$ are the unsteady lift and drag given by

$$
\begin{gathered}
\Delta L=\bar{L}_{x} x+\bar{L}_{u} u+\bar{L}_{w} w \\
\Delta D=\bar{D}_{x} x+\bar{D}_{u} u+\bar{D}_{w} w+x^{\top} \bar{D}_{x^{2}} x+u^{\top} \bar{D}_{u^{2}} u+w^{\top} \bar{D}_{w^{2}} w
\end{gathered}
$$

Using the multi-objective control approach developed in the references, ${ }^{17-20}$ the multi-objective control law is given by

$$
u=K_{x} x+K_{w} w+u_{0}
$$

where

$$
\begin{gathered}
K_{x}=-\bar{R}\left(B^{\top} W+q_{L} \bar{L}_{u}^{\top} \bar{L}_{x}\right) \\
K_{w}=-\bar{R}\left(B^{\top} V+q_{L} \bar{L}_{u}^{\top} \bar{L}_{w}\right) \\
u_{0}=-\bar{R}\left(B^{\top} \bar{\lambda}+\frac{1}{2} q_{D} \bar{D}_{u}^{\top}+q_{L} \bar{L}_{u}^{\top} \Delta \bar{L}\right)
\end{gathered}
$$


We note that $u_{0}$ is a trim control to minimize the drag and load for a new steady-state lift and drag. If we want to control at the current steady-state lift and drag, $u_{0}=0$. The feedback gain $K_{x}$ and disturbance feedforward gain $K_{w}$ are computed from a modified Ricatti equation

$$
W \bar{A} x+\bar{A}^{\top} W x-W B \bar{R}^{-1} B^{\top} W x+\bar{Q} x=0
$$

where

$$
\begin{gathered}
\bar{A}=A-q_{L} B \bar{R}^{-1} \bar{L}_{u}^{\top} \bar{L}_{x} \\
\bar{Q}=Q+q_{D} \bar{D}_{x^{2}}+q_{L} \bar{L}_{x}^{\top}\left(I-q_{L} \bar{L}_{u} \bar{R}^{-1} \bar{L}_{u}^{\top}\right) L_{x} \\
\bar{R}=R+q_{D} \bar{D}_{u^{2}}+q_{L} \bar{L}_{u}^{\top} \bar{L}_{u}
\end{gathered}
$$

along with

$$
V=\left(\bar{A}^{\top}-W B \bar{R}^{-1} B^{\top}\right)^{-1}\left[W\left(q_{L} B \bar{R}^{-1} \bar{L}_{u}^{\top} \bar{L}_{w}-E\right)+q_{L} \bar{L}_{x}^{\top}\left(q_{L} \bar{L}_{u} \bar{R}^{-1} \bar{L}_{u}^{\top}-I\right) \bar{L}_{w}\right]
$$

The trim control $u_{0}$ requires

$$
\begin{aligned}
\bar{\lambda}=\left(\bar{A}^{\top}-W B \bar{R}^{-1} B^{\top}\right)^{-1}\left[W B \bar{R}^{-1}\left(\frac{1}{2} q_{D} \bar{D}_{u}^{\top}+q_{L} \bar{L} \bar{L}_{u}^{\top}\right)\right. & \\
& \left.+\frac{1}{2} q_{D}\left(q_{L} \bar{L}_{x}^{\top} \bar{L}_{u} \bar{R}^{-1} \bar{D}_{u}^{\top}-\bar{D}_{x}^{\top}\right)+q_{L} \bar{L}_{x}^{\top}\left(q_{L} \bar{L}_{u} \bar{R}^{-1} \bar{L}_{u}^{\top}-I\right) \Delta \bar{L}\right]
\end{aligned}
$$

The control implementation requires the state information to be estimated from the sensors using a Kalman filter observer design

$$
\dot{\hat{x}}=A \hat{x}+L(y-\hat{y})+B u+\hat{w}
$$

where $L$ is the Kalman filter observer gain and

$$
\hat{y}=C \hat{x}+D u+E \hat{w}
$$

Then, the multi-objective control is implemented as

$$
u=K_{x} \hat{x}+K_{w} \hat{w}
$$

by ignoring $u_{0}$ since the steady-state lift is not changing. This control law requires estimating $\hat{w}$ which can be through an extended-state implementation, ${ }^{24}$ adaptive estimation, ${ }^{18}$ or a transfer function which requires a physical insight into the disturbance. Alternatively, the disturbance feedforward could be ignored but the load alleviation and drag minimization objectives are still met through the feedback term due to the optimal control formulation. We will demonstrate a multi-objective control law for gust load alleviation without the disturbance feedforward.

Using a simplified ASE frequency-dependent state-space model based on only the $1^{\text {st }}$ bending mode, a control simulation is performed for gust load alleviation at a dynamic pressure of $10 \mathrm{psf}, C_{L}=0.4$, and a sinusoidal gust input at $2 \mathrm{~Hz}$ frequency and $5^{\circ}$ amplitude. We implement a white noise of an amplitude of $1 \mathrm{ft} / s^{2}$ in the sensor signals. The maximum amplitude of the accelerometer signals is about $28 \mathrm{ft} / \mathrm{s}^{2}$, so the signal-to-noise ratio is $28: 1$. We note that we use two accelerometers near the wing tip (see Fig. 12). We use only Flaps 1B and 2B, which are ganged together, for control. Flap 1A is the most effective followed by Flap 2B.

The open-loop and closed-loop responses of the wing are plotted in Figs. 35 - 39. Figure 35 shows that the multi-objective control is able to reduce the unsteady lift substantially by about $50 \%$ from the mean steady-state lift. Figure 36 shows the wing tip displacements. Both open-loop and closed-loop responses produce very similar wing tip displacements which are about $7.8 \mathrm{in}$. We note previously that the generalized NASTRAN stiffness matrix is scaled to produce a mean wing tip displacement of 6 in for $C_{L}=0.4$. So the unsteady lift produces an additional 1.8 in wing tip displacement. Figure 37 shows the control surface deflection which is less than $8^{\circ}$. It is possible that the unsteady lift could be reduced further by increasing the control surface deflection. Figure 38 show the two accelerometer signals in the forward and aft locations. The maximum amplitude of the accelerometers is about $1 \mathrm{~g}$. Figure 39 show the states and their estimates. The states are the generalized displacement $q$ and generalized velocity $\dot{q}$. The state estimation is able to produce good state estimates. 


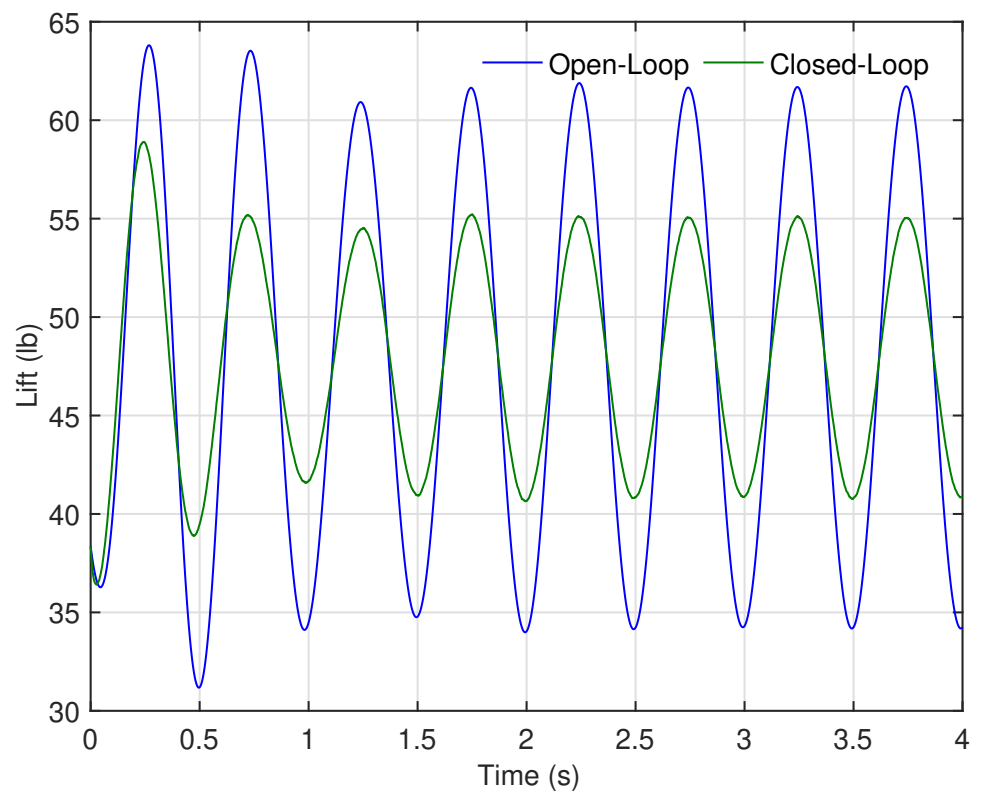

Figure 35. Unsteady Lift Responses

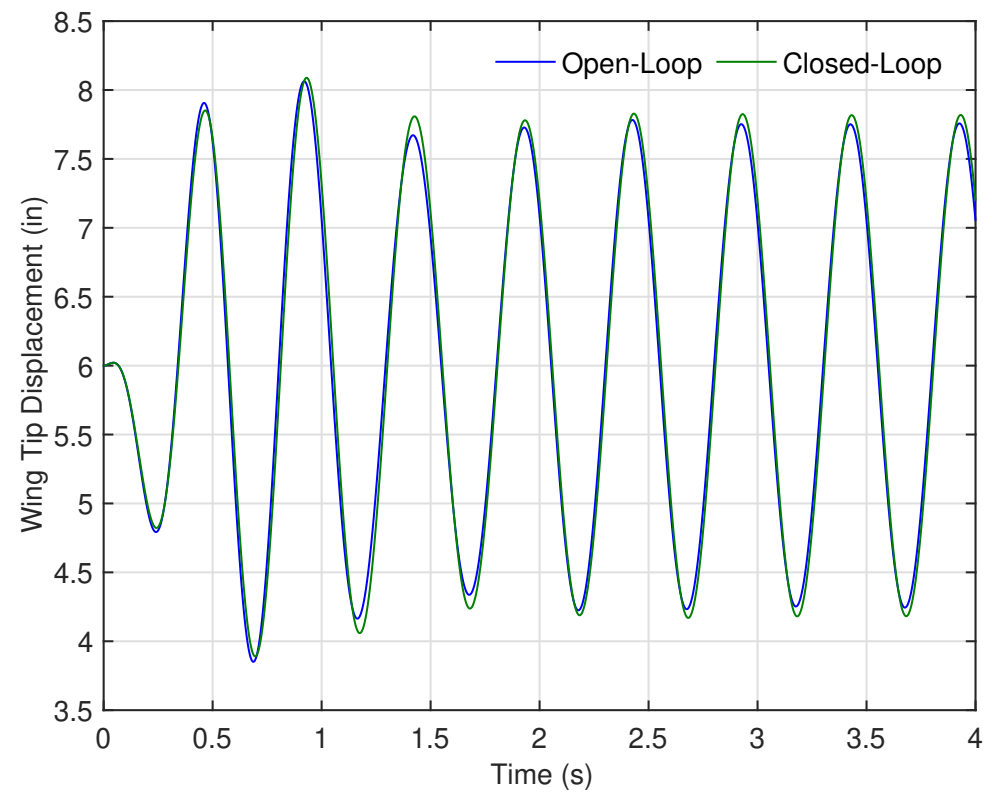

Figure 36. Wing Tip Displacement Response 


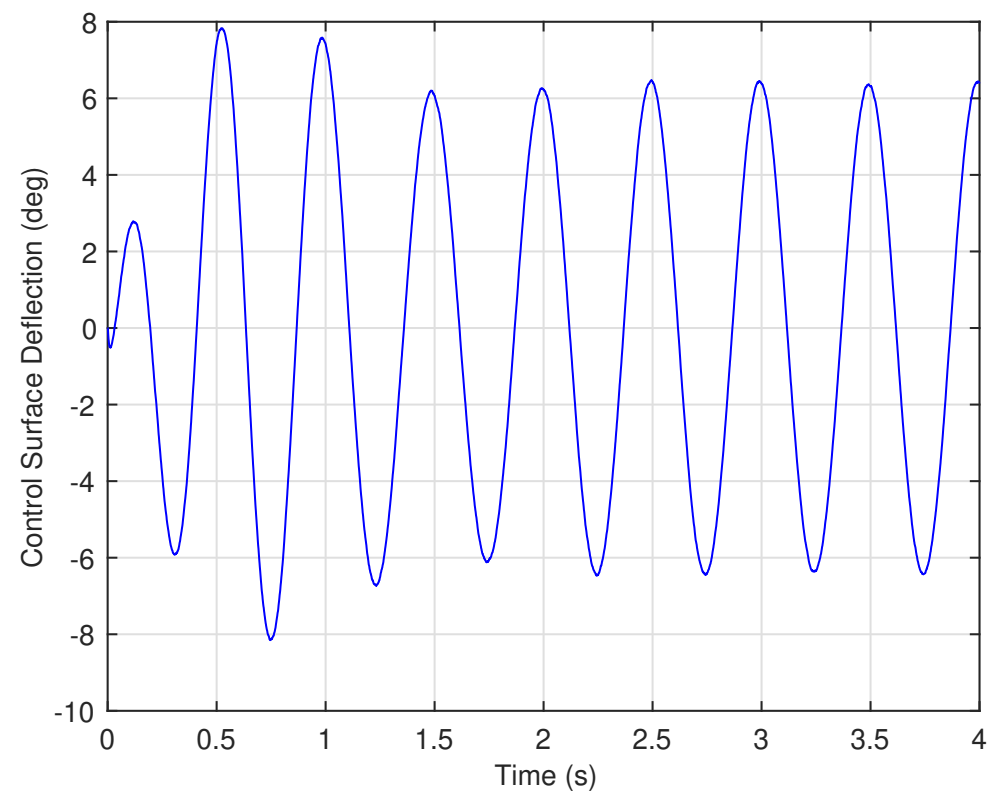

Figure 37. Control Surface Deflection

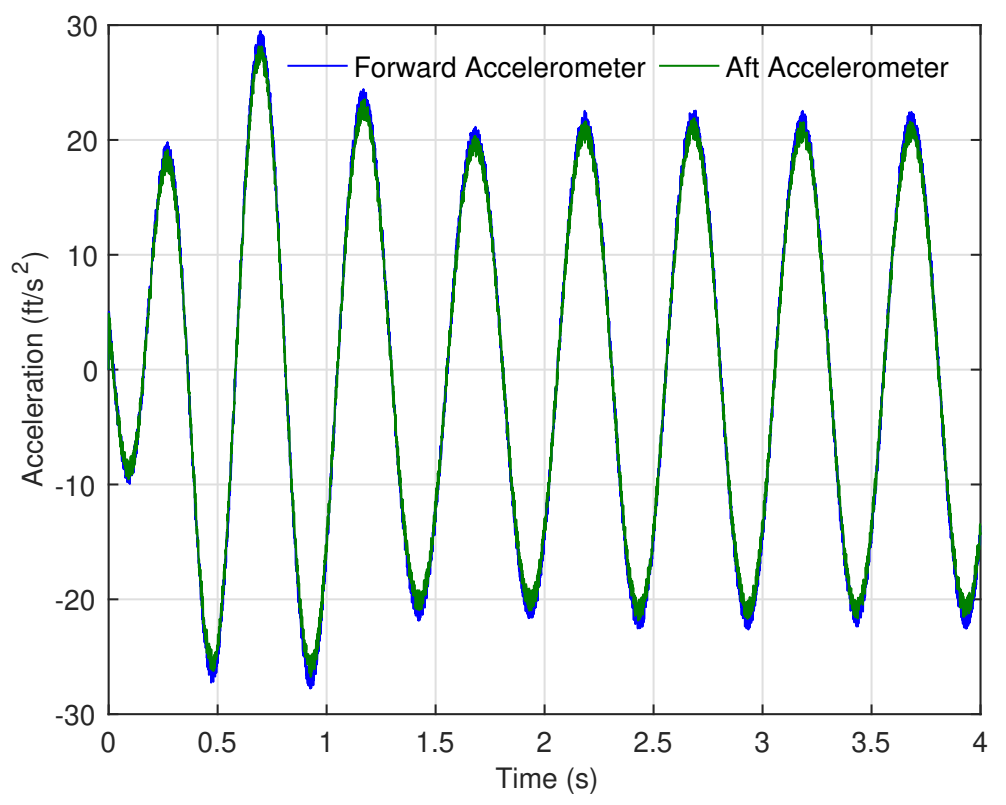

Figure 38. Accelerometer Responses 


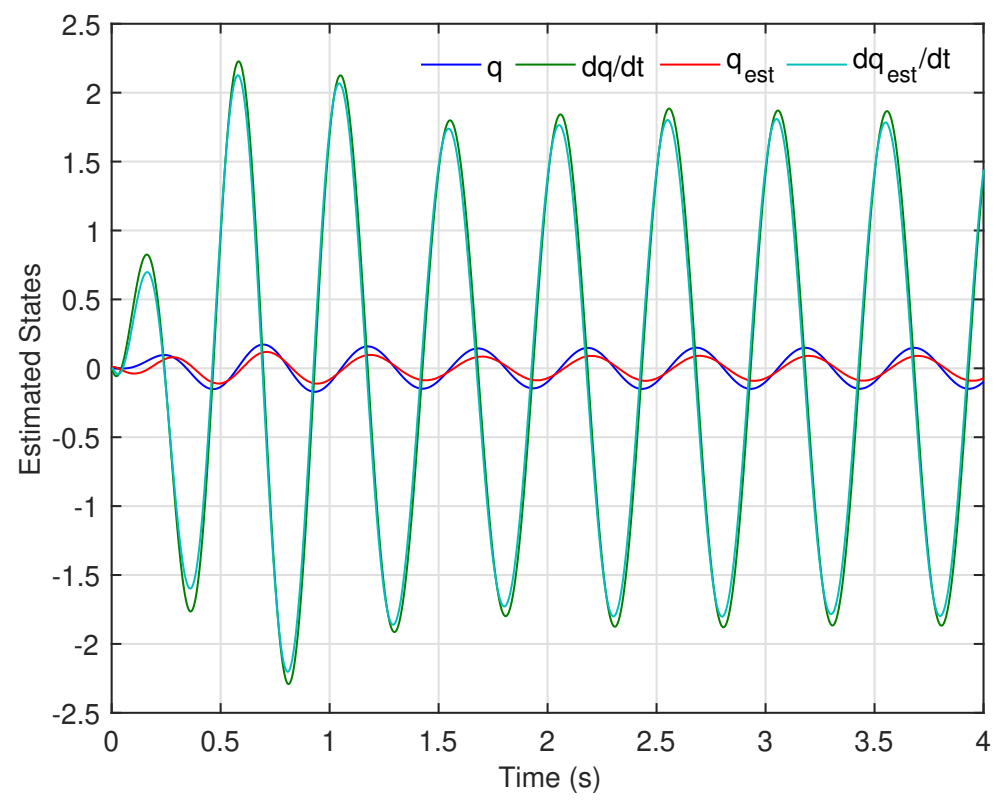

Figure 39. State Estimation

\section{Progress of Test Preparation}

The design and construction of the wing hardware system prove to be a major endeavor. The gust load alleviation wind tunnel test is funded under the NASA SBIR Phase II-X and Phase III contracts with SSCI entitled "Drag Identification \& Reduction Technology (DIRECT) for Elastically Shaped Air Vehicles". The original period of performance was from October 1, 2017 to September 30, 2018 but has been extended until March 20, 2020. Due to the technical issues with the real-time drag optimization wind tunnel test under the SBIR Phase II contract, work on the Phase II-X and Phase III contracts did not start in earnest until after June 2018 when the real-time drag optimization wind tunnel test was completed.

Because of the perceived high risks to the project due to the wing hardware design and the lessons learned from the real-time drag optimization wind tunnel test in 2018, NASA recommended that the majority of the effort should be directed toward supporting the wing hardware design and that an integrated team comprising of SSCI, UW, and NASA be formed to work together on the wing hardware design. The wing design is a complex and challenging activity that involves many new design considerations. The design and fabrication of the wing hardware system was completed in early July 2019. A wind tunnel test was scheduled shortly thereafter starting on July 15,2019 . The plan was to conduct a shake-down test for one week, perform post-test data analysis and ASE model and control update for another week, and then conduct the final test in the third week. Unfortunately, the test was plagued by a litany of serious hardware and software issues that prevent the test objectives from being completed. The test ended on September 27, 2019 without completing the test objectives.

During the wind tunnel test, several issues were identified. Some of the issues are:

- The six accelerometers produce either intermittent or no signals from the very beginning. Some attempted fixes were made including replacing with new accelerometers of the same kind and using modal test $50 \mathrm{~g}$ contact accelerometers which do not have the correct sensitivity. Eventually the issue was found to be due to incorrect wiring and errant soldering. The wiring was repaired and the six accelerometers were brought back into operation. Two of them were installed at the wing tip and then tested in the wind tunnel during the last two weeks and found to produce fairly reasonable signals. The noise level in the accelerometer signals does indicate the need for filtering.

- The servos prove to be problematic. A significant effort was devoted to the servo troubleshooting and tuning. At the very beginning, the servos of Flaps 1A, 1B, and 2A were inoperative. These flaps provide a significant amount of lift for both gust load alleviation and drag optimization. Prior to the test entry, none of the 
servos was tuned and calibrated, so a significant amount of tuning and calibration was required. Some of the servo/flap/potentiometer sub-systems did not repeatably maintain calibration. By the time the test ended, the servos to flap 2B, 3, 4, 5, and 6 were used for data collection, but only 3,4 , and 5 were considered reliable.

- All the strain gauges were declared non-functional and thus abandoned. In lieu, the lift signal from the wind tunnel side wall balance is used to provide a gust load measurement.

- An electromagnetic interference caused by the gust generator drive motor which is installed in close proximity to the data acquisition system prevented the operation of the servos. This was later resolved by installing a copper shielding.

- Data communication between the control computer system and the side wall balance data logger had yet to be worked out. Some initial data were acquired but at a low sampling frequency. The sampling frequency increases to $50 \mathrm{~Hz}$ as required without any major issues. There appears to be a significant latency in the some of the signals collected by the side wall balance data logger. This latency can cause issues with the control experiment. The latency appears to be due to the signal processing in LabView for monitoring the contact accelerometers. Once the wiring was repaired, the contact accelerometers were no longer used and the original data acquisition strategy through the control computer was resumed without routing the signals through the side wall data logger.

- A discrepancy in the angle of attack measurement was discovered. It was found that there are three sources of the angle of attack measurements and they all differ substantially from one another. These sources are alpha encoder, alpha accelerometer, and side wall balance data logger. The alpha encoder is determined to have a scaling error by a scaling factor of 0.89 . The alpha accelerometer is found to be the most accurate but has a $-0.5^{\circ}$ offset error. The side wall balance data logger has a $-1.5^{\circ}$ offset error relative to the alpha accelerometer. The reference zero angle of attack should correspond to the zero incidence angle at the wing root station. This incidence angle is established by the chord line that joins the leading edge and trailing edge of the wing root station when Flaps 1A and 1B are undeflected. When the reference zero angle of attack is physically established, the three angle of attack readings differ from the 'true' trim angle of attack for $C_{L}=0.4$ by as much as $2.6^{\circ}$.

It should be noted that the SSCI/UW/NASA team worked diligently through this trying period with a hope to be able to conduct the control experiment. Nonetheless, the problem seems to be insurmountable and the pressure to end the wind tunnel two-month occupancy began to mount. In the end, SSCI made a decision to have the wing system packaged and shipped to their company facility for them to continue to perform the repair. As of this writing, substantial progress has been made in restoring the intended functionality of the wing system. This includes: correcting the wiring issues, redesigning the string potentiometers to have more extension for the same rotation, upgrading the actuator linkage mechanisms, replacing a micro-controller board for Flap 1A, and removing excess composite material that causes binding or rubbing against the flaps. They anticipate to conduct a re-calibration of the servos with the newly extended range string potentiometers.

On the control law design note, NASA provided to SSCI two ASE state-space models on August 11, 2019. The first model is a simplified frequency-dependent state-space model (see Appendix B1) using only the $1^{\text {st }}$ bending mode. The second model is a four-mode frequency-independent state-space model described in Section III.D. SSCI has been making progress toward completing the control law design. Independently, NASA has been working on the multiobjective control for gust load alleviation and drag optimization with the intent to implement and test the NASA control laws. SSCI recently expressed that the four-mode state-space model with the $D$ matrix in the output causes some difficulty. As a result, NASA sent SSCI on November 20, 2019 the third state-space model which is the same four-mode frequency-independent state-space model but without the $D$ matrix (see Appendix B3). SSCI has since decided that the original four-mode frequency-independent state-space model with the $D$ matrix seems to work best for them. In addition, NASA plans to develop an aerodynamic model to be sent to SSCI and the control law for the drag optimization portion of the test.

The current plan is to conduct the wind tunnel test in late January 2020 for two weeks to complete the test objectives as required by the Phase II-X and Phase III contracts.

\section{Conclusion}

This paper presents an experimental set up of an active control wind tunnel experiment on a flexible wing with the VCCTEF for gust load alleviation and drag optimization. A gust generator is designed and built for the experiment. An ASE model is developed for the gust load alleviation control design. The ASE model uses the NASTRAN mode shapes 
to construct the generalized aerodynamic forces. The gust generator poses a unique aerodynamic modeling problem. Due to the close spacing with the wing, the gust generator and the wing are an aerodynamically coupled system. Both CFD and analytical vortex models are developed to provide physical insight into the gust behavior. An analytical unsteady lift model is developed using both the CFD and vortex models for a sinusoidal gust input. The analytically predicted unsteady lift shows an excellent agreement with the unsteady lift measurement from the wind tunnel side wall balance. Two ASE state-space model formulations are developed. The frequency-dependent formulation is valid at a specific frequency, whereas the frequency-independent formulation is valid over a wider range of frequencies. A multi-objective control for gust load alleviation and drag optimization is developed. A control simulation demonstrates the effectiveness of the multi-objective control.

The first wind tunnel test entry was conducted from July 2019 to September 2019. Several issues with the wing hardware/sensor/software system were encountered. Some of these issues were resolved during the test. The wing system is currently undergoing additional preparation for the next wind tunnel test entry in January 2020.

\section{Acknowledgment}

The authors wish to acknowledge NASA Advanced Air Transport Technologies project for the funding support of this work. The authors also acknowledge the funding support by NASA SBIR program for the wind tunnel test under the NASA SBIR Phase II-X Contract NNX15CA16C and Phase III Contract 80NSSC17C0063. The authors also acknowledge SSCI and UW personnel who support the project.

\section{References}

${ }^{1}$ Nguyen, N., "Elastically Shaped Future Air Vehicle Concept," NASA Innovation Fund Award 2010 Report, October 2010, Submitted to NASA Innovative Partnerships Program, http://ntrs.nasa.gov/archive/nasa

/casi.ntrs.nasa.gov/20110023698.pdf

${ }^{2}$ Nguyen, N. and Urnes, J., "Aeroelastic Modeling of Elastically Shaped Aircraft Concept via Wing Shaping Control for Drag Reduction," AIAA Atmospheric Flight Mechanics Conference, AIAA-2012-4642, August 2012.

${ }^{3}$ Nguyen, N., Trinh, K., Reynolds, K., Kless, J., Aftosmis, M., Urnes, J., and Ippolito, C., "Elastically Shaped Wing Optimization and Aircraft Concept for Improved Cruise Efficiency," 51st AIAA Aerospace Sciences Meeting, AIAA-2013-0141, January 2013.

${ }^{4}$ Boeing Report No. 2012X0015, "Development of Variable Camber Continuous Trailing Edge Flap System," Submitted to NASA, October 4, 2012.

${ }^{5}$ Urnes, J., Nguyen, N., Ippolito, C., Totah, J., Trinh, K., and Ting, E., "A Mission Adaptive Variable Camber Flap Control System to Optimize High Lift and Cruise Lift to Drag Ratios of Future N+3 Transport Aircraft," 51st AIAA Aerospace Sciences Meeting, AIAA-2013-0214, January 2013.

${ }^{6}$ Boeing Report No. 2014X0030, "Development of Variable Camber Continuous Trailing Edge Flap System for B757 Configured with a More Flexible Wing," Submitted to NASA, September 27, 2014.

${ }^{7}$ Urnes, J., Morris, C., Sheahan, J., Dykman, J., and Klingman, D., "Control System Design for a Variable Camber Continuous Trailing Edge Flap System on an Elastic Wing," 55th AIAA/ASME/ASCE/AHS/ASC Structures, Structural Dynamics, and Materials Conference, AIAA-20140835, January 2014.

${ }^{8}$ Dykman, J., Truong, H., and Urnes, J., "Active Control for Elastic Wing Structure Dynamic Modes," 56th AIAA/ASCE/AHS/ASC Structures, Structural Dynamics, and Materials Conference, AIAA-2015-1842, January 2015.

${ }^{9}$ Ferrier, Y., Nguyen, N., and Ting, E., "Real-Time Adaptive Least-Squares Drag Minimization for Performance Adaptive Aeroelastic Wing," 34th AIAA Applied Aerodynamics Conference, AIAA-2016-3567, June 2016.

${ }^{10}$ Ting, E., Chaparro, D., Nguyen, N., and Fujiwara, G., "Optimization of Variable Camber Continuous Trailing Edge Flap Configuration for Drag Reduction,” AIAA Journal of Aircraft, Vol. 55, No. 6, pp. 2217-2239, November 2018.

${ }^{11}$ Nguyen, N., Precup, N., Urnes, J., Nelson, C., Lebofsky, S., Ting, E., and Livne, E., "Experimental Investigation of a Flexible Wing with a Variable Camber Continuous Trailing Edge Flap Design,” 32nd AIAA Applied Aerodynamics, AIAA 2014-2441, June 2014.

${ }^{12}$ Precup, N., Mor, M., and Livne, E., "Design, Construction, and Tests of an Aeroelastic Wind Tunnel Model of a Variable Camber Continuous Trailing Edge Flap (VCCTEF) Concept Wing," 32nd AIAA Applied Aerodynamics Conference, AIAA-2014-2442, June 2014.

${ }^{13}$ Nguyen, N., Precup, N., Livne, E., Urnes, J., Dickey, E., Nelson, C., Chiew, J., Rodriguez, D., Ting, E., and Lebofsky, S., "Wind Tunnel Investigation of a Flexible Wing High-Lift Configuration with a Variable Camber Continuous Trailing Edge Flap Design," 33rd AIAA Applied Aerodynamics Conference, AIAA-2015-2417, June 2015.

${ }^{14}$ Precup, N., Mor, M., and Livne, E., "The Design, Construction, and Tests of a Concept Aeroelastic Wind Tunnel Model of a High-Lift Variable Camber Continuous Trailing Edge Flap ( HL-VCCTEF) Wing Configuration,” 56th AIAA/ASCE/AHS/ASC Structures, Structural Dynamics, and Materials Conference, AIAA-2015-1406, January 2015.

${ }^{15}$ Precup, N., Mundt, T., Mor, M., Livne, E., "An Active Variable Camber Continuous Trailing Edge Flapped Wing Wind Tunnel Model for Aeroelastic "In-Flight" Shape Optimization Tests," AIAA Multidisciplinary Analysis and Optimization Conference, AIAA-2018-3106, June 2018.

${ }^{16}$ Nguyen, N., Cramer, N., Hashemi, K., Drew, M., Wise, R., Boskovic, J., Mundt, T., Precup, N., and Livne, E., "Real-Time Adaptive Drag Minimization Wind Tunnel Investigation of a Flexible Wing with Variable Camber Continuous Trailing Edge Flap System," AIAA Applied Aerodynamic Conference, AIAA-2019-3156, June 2019.

${ }^{17}$ Drew, M., Hashemi, K., Cramer, N., and Nguyen, N., "Multi-Objective Optimal Control of the 6-DoF Aeroservoelastic Common Research Model with Aspect Ratio 13.5 Wing," AIAA Structural Dynamics Conference, AIAA-2019-0220, January 2019. 
${ }^{18}$ Nguyen, N., Hashemi, K., and Drew, M., "Multi-Objective Adaptive Control for Load Alleviation and Drag Minimization of Flexible Aircraft," AIAA Guidance, Navigation, and Control Conference, AIAA-2018-0622, January 2018.

${ }^{19}$ Nguyen, N., Ting, E., Chaparro, D., Drew, M., and Swei, S., "Multi-Objective Flight Control for Drag Minimization and Load Alleviation of High-Aspect Ratio Flexible Wing Aircraft," 58th AIAA/ASME/ASCE/AHS/SC Structures, Structural Dynamics, and Materials Conference, AIAA-2017-1589, January 2017.

${ }^{20}$ Nguyen, N. and Tal, E., "A Multi-Objective Flight Control Approach for Performance Adaptive Aeroelastic Wing," 56th AIAA/ASME/ASCE/AHS/SC Structures, Structural Dynamics, and Materials Conference, AIAA-2015-1843, January 2015. Vassberg, J., Dehaan M., Rivers, M., and Wahls, R., "Development of a Common Research Model for Applied CFD Validation Studies," 26th AIAA Applied Aerodynamics Conference, AIAA-2008-6919, August 2008.

${ }^{21}$ Theodorsen, T., "General Theory of Aerodynamic Instability and the Mechanism of Flutter," NACA Report 496, 1949.

${ }^{25}$ Anderson, J., Fundamentals of Aerodynamics, McGraw-Hill, New York, 2001

${ }^{22}$ Bisplinghoff, R. L., Ashley, H., and Halfman, R. L., Aeroelasticity, Dover Publications, Inc., New York, 1996.

${ }^{23}$ Jones, R. T., "Operational Treatment of the Nonuniform-Lift Theory in Airplane Dynamics," NACA TN 667, 1938.

${ }^{24}$ Ferrier, Y., Nguyen, N., Ting, E., Chaparro, D., Wang, X., de Visser, C., and Chu, Q., "Active Gust Load Alleviation of High-Aspect Ratio Flexible Wing Aircraft," AIAA Guidance, Navigation, and Control Conference, AIAA-2018-0620, January 2018.

\section{Appendix A}


1. The lift and pitching moment sensitivities for Eqs. (8) and (9) are

$$
\begin{gathered}
l_{0}=q_{\infty} c c_{L_{0}} \cos \Lambda \\
l_{\alpha}=q_{\infty} c c_{L_{\alpha}} \cos \Lambda \\
l_{q}^{*}=q_{\infty} c c_{L_{\alpha}} \cos \Lambda\left(\Psi \cos \Lambda-\Phi^{\prime} \sin \Lambda\right) \\
l_{\dot{q}}^{*}=\frac{q_{\infty}}{V_{\infty}} c c_{L_{\alpha}} \cos \Lambda\left(e_{c} \Psi \cos \Lambda-\Phi\right) \\
l_{\dot{q}}=\frac{\pi}{2} \frac{q_{\infty}}{V_{\infty}} c^{2} \Psi \cos ^{2} \Lambda \\
l_{\ddot{q}}=\frac{\pi}{2} \frac{q_{\infty}}{V_{\infty}^{2}} c^{2} \cos \Lambda\left(e_{m} \Psi \cos \Lambda-\Phi\right) \\
m_{0}=q_{\infty} c\left(c_{m_{a c}}+e c_{L_{0}}\right) \cos \Lambda \sin \Lambda \\
m_{\alpha}=q_{\infty} c e c_{L_{\alpha}} \cos \Lambda \sin \Lambda \\
m_{q}^{*}=q_{\infty} c e c_{L_{\alpha}} \cos \Lambda \sin \Lambda\left(\Psi \cos \Lambda-\Phi^{\prime} \sin \Lambda\right) \\
m_{\dot{q}}^{*}=\frac{q_{\infty}}{V_{\infty}} c e c_{L_{\alpha}} \cos \Lambda \sin \Lambda\left(e_{c} \Psi \cos \Lambda-\Phi\right) \\
m_{\dot{q}}=-\frac{\pi}{2} \frac{q_{\infty}}{V_{\infty}} c^{2} e_{c} \Psi \cos { }^{2} \Lambda \sin \Lambda \\
m_{\ddot{q}}=-\frac{\pi}{2} \frac{q_{\infty}}{V_{\infty}^{2}} c^{2} e_{m} \cos \Lambda \sin \Lambda\left(e_{m} \Psi \cos \Lambda-\Phi\right)-\frac{\pi}{64} \frac{q_{\infty}}{V_{\infty}^{2}} c^{4} \Psi \cos ^{2} \Lambda \sin \Lambda
\end{gathered}
$$

2. The generalized aerodynamic force sensitivities for Eqs. (10) and (11) are

$$
\begin{gathered}
F_{0}=\int_{0}^{L}\left(\Phi^{\top} l_{0}-\Phi^{\prime \top} m_{0}\right) d x \\
F_{\alpha}=\int_{0}^{L}\left(\Phi^{\top} l_{\alpha}-\Phi^{\prime} m_{\alpha}\right) d x \\
M_{a}=-\int_{0}^{L}\left(\Phi^{\top} l_{\ddot{q}}-\Phi^{\prime \top} m_{\ddot{q}}\right) d x \\
C_{a}=-\int_{0}^{L}\left(\Phi^{\top} l_{\dot{q}}-\Phi^{\prime \top} m_{\dot{q}}\right) d x \\
C_{a}^{*}=-\int_{0}^{L}\left(\Phi^{\top} l_{\dot{q}}^{*}-\Phi^{\prime} m_{\dot{q}}^{*}\right) d x \\
K_{a}^{*}=-\int_{0}^{L}\left(\Phi^{\top} l_{q}^{*}-\Phi^{\prime \top} m_{q}^{*}\right) d x
\end{gathered}
$$

3. The total lift and pitching moment sensitivities for Eqs. (12) and (13) are

$$
\begin{aligned}
L_{q}^{*} & =\int_{0}^{L} l_{q}^{*} d x \\
L_{\dot{q}}^{*} & =\int_{0}^{L} l_{\dot{q}}^{*} d x \\
L_{\dot{q}} & =\int_{0}^{L} l_{\dot{q}} d x \\
L_{\ddot{q}} & =\int_{0}^{L} l_{\ddot{q}} d x \\
M_{q}^{*} & =\int_{0}^{L} e_{p} l_{q}^{*} d x
\end{aligned}
$$




$$
\begin{aligned}
M_{\dot{q}}^{*} & =\int_{0}^{L} e_{p} m_{\dot{q}}^{*} d x \\
M_{\dot{q}} & =\int_{0}^{L} m_{\dot{q}} d x \\
M_{\ddot{q}} & =\int_{0}^{L} M_{\ddot{q}} d x
\end{aligned}
$$

where $e_{p}$ is the distance between the pitch axis and the quarter chord, positive when the pitch axis is behind the quarter chord.

4. The lift and pitching moment coefficient sensitivities due to the flap motion for Eqs. (14) and (15) are

$$
\begin{aligned}
& c_{L_{\delta}}=\frac{c_{L_{\alpha}} T_{10}}{\pi}=\frac{c_{L_{\alpha}}}{\pi}\left(\cos ^{-1} c^{*}+\sqrt{1-c^{* 2}}\right) \\
& c_{L_{\dot{\delta}}}=\frac{c_{L_{\alpha}} T_{11}}{2 \pi}=\frac{c_{L_{\alpha}}}{2 \pi}\left[\left(1-2 c^{*}\right) \cos ^{-1} c^{*}+\left(2-c^{*}\right) \sqrt{1-c^{* 2}}\right]=\frac{c_{L_{\delta}} T_{11}}{2 T_{10}} \\
& \bar{c}_{L_{\dot{\delta}}}=-T_{4}=\cos ^{-1} c^{*}-c^{*} \sqrt{1-c^{* 2}} \\
& \bar{c}_{L_{\ddot{\delta}}}=-T_{1}=-c^{*} \cos ^{-1} c^{*}+\frac{\left(2+c^{* 2}\right) \sqrt{1-c^{* 2}}}{3} \\
& c_{m_{\delta}}=-\frac{T_{4}+T_{10}}{2}=-\frac{\left(1+c^{*}\right) \sqrt{1-c^{* 2}}}{2} \\
& c_{m_{\dot{\delta}}}=-\frac{T_{1}-T_{8}-\left(c^{*}-a\right) T_{4}+\frac{T_{11}}{2}}{2}=-\frac{\left(\frac{1}{2}-a\right) \cos ^{-1} c^{*}+\left(\frac{2}{3}-\frac{c^{*}}{2}+a c^{*}-\frac{2 c^{* 2}}{3}\right) \sqrt{1-c^{* 2}}}{2} \\
& c_{m_{\ddot{\delta}}}=\frac{T_{7}+\left(c^{*}-a\right) T_{1}}{2}=\frac{-\left(\frac{1}{8}+a c^{*}\right) \cos ^{-1} c^{*}+\left(\frac{2 a}{3}+\frac{5 c^{*}}{24}+\frac{a c^{* 2}}{3}-\frac{c^{* 3}}{12}\right) \sqrt{1-c^{* 2}}}{2}
\end{aligned}
$$

where $c^{*}=1-\frac{2 c_{f}}{c}$ and $c_{f}$ is the flap chord, $a=\frac{2 e}{c}-\frac{1}{2}$, and $T_{i}, i=1 \ldots, 11$, are given in NACA Report $496 .^{21}$

5. The lift and pitching moment sensitivities due to the flap motion for Eqs. (16) and (17) are

$$
\begin{gathered}
l_{\bar{\delta}}=q_{\infty} c c_{L_{\delta}} \bar{\delta} \cos \Lambda \\
l_{\delta}^{*}=q_{\infty} c c_{L_{\delta}} \cos \Lambda \\
l_{\dot{\delta}}^{*}=\frac{q_{\infty}}{2 V_{\infty}} c^{2} c_{L_{\dot{\delta}}} \cos \Lambda \\
l_{\dot{\delta}}=\frac{q_{\infty}}{2 V_{\infty}} c^{2} \bar{c}_{L_{\dot{\delta}}} \cos \Lambda \\
l_{\ddot{\delta}}=\frac{q_{\infty}}{4 V_{\infty}^{2}} c^{3} \bar{c}_{L_{\ddot{\delta}}} \cos \Lambda \\
m_{\bar{\delta}}=q_{\infty} c c_{m_{\delta}} \bar{\delta} \cos \Lambda \sin \Lambda \\
m_{\delta}^{*}=q_{\infty} c e c_{L_{\delta}} \cos \Lambda \sin \Lambda \\
m_{\delta}=q_{\infty} c^{2} c_{m_{\delta}} \cos \Lambda \sin \Lambda \\
m_{\dot{\delta}}^{*}=\frac{q_{\infty}}{2 V_{\infty}} c^{2} e c_{L_{\dot{\delta}}} \cos \Lambda \sin \Lambda \\
m_{\dot{\delta}}=\frac{q_{\infty}}{2 V_{\infty}} c^{3} c_{m_{\dot{\delta}}} \cos \Lambda \sin \Lambda \\
m_{\ddot{\delta}}=\frac{q_{\infty}}{4 V_{\infty}^{2}} c^{4} c_{m_{\ddot{\delta}}} \cos \Lambda \sin \Lambda
\end{gathered}
$$


6. The generalized aerodynamic force sensitivities due to the flap motion for Eqs. (18) and (19) are

$$
\begin{gathered}
F_{\delta_{i}}=\int_{a_{i}}^{b_{i}}\left(-\Phi^{\top} m_{\delta_{i}}\right) d x \\
F_{\delta_{i}}^{*}=\int_{a_{i}}^{b_{i}}\left(\Phi^{\top} l_{\delta_{i}}^{*}-\Phi^{\prime \top} m_{\delta_{i}}^{*}\right) d x \\
F_{\dot{\delta}_{i}}=\int_{a_{i}}^{b_{i}}\left(\Phi^{\top} l_{\dot{\delta}_{i}}-\Phi^{\prime} m_{\dot{\delta}_{i}}\right) d x \\
F_{\dot{\delta}_{i}}^{*}=\int_{a_{i}}^{b_{i}}\left(\Phi^{\top} l_{\dot{\delta}_{i}}^{*}-\Phi^{\prime \top} m_{\dot{\delta}_{i}}^{*}\right) d x \\
F_{\ddot{\delta}_{i}}=\int_{0}^{L}\left(\Phi^{\top} l_{\ddot{\delta}_{i}}-\Phi^{\top} m_{\ddot{\delta}_{i}}\right) d x
\end{gathered}
$$

where $a_{i}$ and $b_{i}$ are the pitch axis $y$ coordinates of the flap edges along the wing span.

7. The total lift and pitching moment sensitivities due to the flap motion for Eqs. (20) and (21) are

$$
\begin{gathered}
L_{\delta}^{*}=q_{\infty} S C_{L_{\delta}} \\
L_{\dot{\delta}}^{*}=\frac{q_{\infty}}{2 V_{\infty}} S \bar{c} C_{L_{\dot{\delta}}} \\
L_{\dot{\delta}}=\frac{q_{\infty}}{2 V_{\infty}} S \bar{c} \bar{C}_{L_{\dot{\delta}}} \\
L_{\ddot{\delta}}=\frac{q_{\infty}}{4 V_{\infty}^{2}} S \bar{c}^{2} \bar{C}_{L_{\ddot{\delta}}} \\
M_{\delta}^{*}=q_{\infty} S \bar{e}_{\delta} C_{L_{\delta}} \\
M_{\dot{\delta}}^{*}=\frac{q_{\infty}}{2 V_{\infty}} S \bar{c} \bar{e}_{\dot{\delta}} C_{L_{\dot{\delta}}} \\
M_{\delta}=q_{\infty} S \bar{c} C_{m_{\delta}} \\
M_{\dot{\delta}}=\frac{q_{\infty}}{2 V_{\infty}} S \bar{c}^{2} C_{m_{\dot{\delta}_{i}}} \\
M_{\ddot{\delta}}=\frac{q_{\infty}}{4 V_{\infty}^{2}} S \bar{c}^{3} C_{m_{\ddot{\delta}_{i}}}
\end{gathered}
$$

where the lift and pitching moment coefficient sensititivies due to the flap motion are given by

$$
\begin{gathered}
C_{L_{\delta_{i}}}=\frac{1}{S} \int_{a_{i}}^{b_{i}} c c_{L_{\delta_{i}}} d y \\
C_{L_{\dot{\delta}_{i}}}=\frac{1}{S \bar{c}} \int_{a_{i}}^{b_{i}} c^{2} c_{L_{\dot{\delta}_{i}}} d y \\
\bar{C}_{L_{\dot{\delta}_{i}}}=\frac{1}{S \bar{c}} \int_{a_{i}}^{b_{i}} c^{2} \bar{c}_{{L_{\delta_{i}}}_{\delta_{i}}} d y \\
\bar{C}_{L_{\tilde{\delta}_{i}}}=\frac{1}{S \bar{c}^{2}} \int_{a_{i}}^{b_{i}} c^{3} \bar{c}_{L_{\tilde{\delta}_{i}}} d y \\
C_{m_{\delta_{i}}}=\frac{1}{S \bar{c}} \int_{a_{i}}^{b_{i}} c^{2} c_{m_{\delta_{i}}} d y \\
C_{m_{\dot{\delta}_{i}}}=\frac{1}{S \bar{c}^{2}} \int_{a_{i}}^{b_{i}} c^{3} c_{m_{\dot{\delta}_{i}}} d y \\
C_{m_{\ddot{\delta}_{i}}}=\frac{1}{S \bar{c}^{3}} \int_{a_{i}}^{b_{i}} c^{4} c_{m_{\tilde{\delta}_{i}}} d y
\end{gathered}
$$

and

$$
\begin{gathered}
\bar{e}_{\delta_{i}}=\frac{1}{S C_{L_{\delta_{i}}}} \int_{a_{i}}^{b_{i}} c e_{p} c_{L_{\delta_{i}}} d y \approx \frac{1}{S} \int_{a_{i}}^{b_{i}} c e_{p} d y \\
\bar{e}_{\dot{\delta}_{i}}=\frac{1}{S \bar{c} C_{L_{\delta_{i}}}} \int_{a_{i}}^{b_{i}} c^{2} e_{p} c_{L_{\dot{\delta}_{i}}} d y \approx \frac{1}{S} \int_{a_{i}}^{b_{i}} c e_{p} d y
\end{gathered}
$$




\section{Appendix B}

1. We formulate the frequency-dependent ASE state-space model by assuming $q=|q| e^{i \omega t}$ and $\delta=|\delta| e^{i \omega t}$ at a specific frequency. Then, the equation of motion is expressed as

$$
\begin{aligned}
{\left[M+M_{a}+\right.} & \left.\frac{G(k)}{\omega} C_{a}^{*}\right] \ddot{q}+\left[C+C_{a}+F(k) C_{a}^{*}+\frac{G(k)}{\omega} K_{a}^{*}\right] \dot{q}+\left[K+F(k) K_{a}^{*}\right] q=F_{0}+F_{\alpha} \alpha+F_{\delta} \bar{\delta} \\
& +\left[F_{\delta}+F(k) F_{\delta}^{*}\right] \delta+\left[F_{\dot{\delta}}+F(k) F_{\delta}^{*}+\frac{G(k)}{\omega} F_{\delta}^{*}\right] \dot{\delta}+\left[F_{\ddot{\delta}}+\frac{G(k)}{\omega} F_{\dot{\delta}}^{*}\right] \ddot{\delta}+F_{\theta_{v}} \theta_{v}+F_{\dot{\theta}_{v}} \dot{\theta}_{v}
\end{aligned}
$$

We consider two frequency-dependent ASE state-space models: 1) model with output independent of the control $(D=0)$ and 2) model with output dependent on the control $u(D \neq 0)$.

(a) Output Independent of Control

The actuator dynamics are modeled as

$$
\ddot{\delta}+2 \zeta_{a} \omega_{a} \dot{\delta}+\omega_{a}^{2} \delta=-d k_{p}\left(\delta-\delta_{c}\right)-d k_{i} \int_{0}^{t}\left(\delta-\delta_{c}\right) d \tau-d k_{d}\left(\dot{\delta}-\dot{\delta}_{c}\right)
$$

Then, the actuator model is written as

$$
\dddot{\delta}+\left(2 \zeta_{a} \omega_{a}+d k_{d}\right) \ddot{\delta}+\left(\omega_{a}^{2}+d k_{p}\right) \dot{\delta}+d k_{i} \delta=d k_{i} u
$$

where the command vector $\delta_{c}$ to the flap deflections is computed from the control command vector

$$
k_{d} \ddot{\delta}_{c}+k_{p} \dot{\delta}_{c}+k_{i} \delta_{c}=k_{i} u
$$

Let $x=\left[\begin{array}{ccccc}q & \dot{q} & \delta & \dot{\delta} & \ddot{\delta}\end{array}\right]^{\top}$. Then, the state-space model is described by the following matrices:

$$
\begin{aligned}
& A=\left[\begin{array}{ccccc}
0 & I & 0 & 0 & 0 \\
-\bar{M}^{-1} \bar{K} & -\bar{M}^{-1} \bar{C} & \bar{M}^{-1} \bar{F}_{\delta} & \bar{M}^{-1} \bar{F}_{\dot{\delta}} & \bar{M}^{-1} \bar{F}_{\ddot{\delta}} \\
0 & 0 & 0 & I & 0 \\
0 & 0 & 0 & 0 & I \\
0 & 0 & -d k_{i} & -\left(\omega_{a}^{2}+d k_{p}\right) & -\left(2 \zeta_{a} \omega_{a}+d k_{d}\right)
\end{array}\right] \\
& B=\left[\begin{array}{c}
0 \\
0 \\
0 \\
0 \\
d k_{i}
\end{array}\right] \\
& E=\left[\begin{array}{cc}
0 & 0 \\
\bar{M}^{-1} F_{\theta_{v}} & \bar{M}^{-1} F_{\dot{\theta}_{v}} \\
0 & 0 \\
0 & 0 \\
0 & 0
\end{array}\right] \\
& \bar{M}=M+M_{a}+\frac{G(k)}{\omega} C_{a}^{*} \\
& \bar{C}=C+C_{a}+F(k) C_{a}^{*}+\frac{G(k)}{\omega} K_{a}^{*} \\
& \bar{K}=K+F(k) K_{a}^{*} \\
& \bar{F}_{\delta}=F_{\delta}+F(k) F_{\delta}^{*}
\end{aligned}
$$




$$
\begin{gathered}
\bar{F}_{\dot{\delta}}=F_{\dot{\delta}}+F(k) F_{\dot{\delta}}^{*}+\frac{G(k)}{\omega} F_{\delta}^{*} \\
\bar{F}_{\ddot{\delta}}=F_{\ddot{\delta}}+\frac{G(k)}{\omega} F_{\dot{\delta}}^{*}
\end{gathered}
$$

The output vector is described by

$$
y=C x+F w
$$

where

$$
\begin{aligned}
& C=\left[\Phi\left(x_{a}\right)+e_{a} \Psi\left(x_{a}\right)\right] I_{\ddot{q}} A \\
& F=\left[\Phi\left(x_{a}\right)+e_{a} \Psi\left(x_{a}\right)\right] I_{\ddot{q}} E
\end{aligned}
$$

$I_{\ddot{q}}=\left[\begin{array}{lllll}0 & I & 0 & 0 & 0\end{array}\right]$, and we note that $I_{\ddot{q}} B=0$.

The unsteady lift is described by

$$
L=\bar{L}+\bar{L}_{x} x+\bar{L}_{w} w
$$

where

$$
\begin{aligned}
& \bar{L}=q_{\infty} S\left(C_{L_{0}}+C_{L_{\alpha}} \alpha+C_{L_{\delta}} \bar{\delta}\right) \\
& \bar{L}_{x}=L_{x}+\bar{L}_{\ddot{q}} I_{\ddot{q}} A \\
& \bar{L}_{w}=L_{w}+\bar{L}_{\ddot{q}} I_{\ddot{q}} E \\
& L_{x}=\left[\begin{array}{lllll}
\bar{L}_{q} & \bar{L}_{\dot{q}} & \bar{L}_{\delta} & \bar{L}_{\dot{\delta}} & \bar{L}_{\ddot{\delta}}
\end{array}\right] \\
& L_{w}=\left[\begin{array}{ll}
L_{\theta_{v}} & L_{\dot{\theta}_{v}}
\end{array}\right] \\
& \bar{L}_{q}=F(k) L_{q}^{*} \\
& \bar{L}_{\dot{q}}=L_{\dot{q}}+F(k) L_{\dot{q}}^{*}+\frac{G(k)}{\omega} L_{q}^{*}+\Delta L_{\dot{q}} \\
& \bar{L}_{\ddot{q}}=L_{\ddot{q}}+\frac{G(k)}{\omega} L_{\dot{q}}^{*}+\Delta L_{\ddot{q}} \\
& \bar{L}_{\delta}=F(k) L_{\delta}^{*} \\
& \bar{L}_{\dot{\delta}}=L_{\dot{\delta}}+F(k) L_{\dot{\delta}}^{*}+\frac{G(k)}{\omega} L_{\delta}^{*} \\
& \bar{L}_{\ddot{\delta}}=L_{\ddot{\delta}}+\frac{G(k)}{\omega} L_{\dot{\delta}}^{*}
\end{aligned}
$$

(b) Output Dependent on Control

Let $x=\left[\begin{array}{cccc}q & \dot{q} & \delta & \dot{\delta}\end{array}\right]^{\top}$. Then, the state-space model is described by the following matrices:

$$
\begin{gathered}
A=\left[\begin{array}{cccc}
0 & I & 0 & 0 \\
-\bar{M}^{-1} \bar{K} & -\bar{M}^{-1} \bar{C} & \bar{M}^{-1} \bar{F}_{\delta} & \bar{M}^{-1} \bar{F}_{\dot{\delta}} \\
0 & 0 & 0 & I \\
0 & 0 & -\omega_{a}^{2} & -2 \zeta_{a} \omega_{a}
\end{array}\right] \\
B=\left[\begin{array}{cc}
0 & \\
\bar{M}^{-1} \bar{F}_{\ddot{\delta}} d \\
0 \\
& d
\end{array}\right] \\
E=\left[\begin{array}{cc}
0 & 0 \\
\bar{M}^{-1} F_{\theta_{v}} & \bar{M}^{-1} F_{\dot{\theta}_{v}} \\
0 & 0 \\
0 & 0
\end{array}\right] \\
41 \text { of } 43
\end{gathered}
$$


where $(\bar{M}, \bar{C}, \bar{K})$ are from Eqs. (192) - (194), and $\left(\bar{F}_{\delta}, \bar{F}_{\dot{\delta}}\right)$ are replaced by

$$
\begin{gathered}
\bar{F}_{\delta}=F_{\delta}+F(k) F_{\delta}^{*}-\left[F_{\ddot{\delta}}+\frac{G(k)}{\omega} F_{\dot{\delta}}^{*}\right] \omega_{a}^{2} \\
\bar{F}_{\dot{\delta}}=F_{\dot{\delta}}+F(k) F_{\dot{\delta}}^{*}+\frac{G(k)}{\omega} F_{\delta}^{*}-\left[F_{\ddot{\delta}}+\frac{G(k)}{\omega} F_{\dot{\delta}}^{*}\right] 2 \zeta_{a} \omega_{a}
\end{gathered}
$$

The output vector is described by

$$
y=C x+D u+F w
$$

where

$$
\begin{aligned}
& C=\left[\Phi\left(x_{a}\right)+e_{a} \Psi\left(x_{a}\right)\right] I_{\ddot{q}} A \\
& D=\left[\Phi\left(x_{a}\right)+e_{a} \Psi\left(x_{a}\right)\right] I_{\ddot{q}} B \\
& F=\left[\Phi\left(x_{a}\right)+e_{a} \Psi\left(x_{a}\right)\right] I_{\ddot{q}} E
\end{aligned}
$$

and $I_{\ddot{q}}=\left[\begin{array}{cccc}0 & I & 0 & 0\end{array}\right]$.

The unsteady lift is given by

$$
L=\bar{L}+\bar{L}_{x} x+\bar{L}_{u} u+\bar{L}_{w} w
$$

where $\left(\bar{L}_{x}, \bar{L}_{w}\right)$ are given by Eqs. (203), (204),

$$
\bar{L}_{u}=L_{u}+\bar{L}_{\ddot{q}} I_{\ddot{q}} B
$$

$L_{u}$ is given by Eq. (95), and $\left(L_{x}, \bar{L}_{\delta}, \bar{L}_{\dot{\delta}}\right)$ are replaced by

$$
\begin{gathered}
L_{x}=\left[\begin{array}{llll}
\bar{L}_{q} & \bar{L}_{\dot{q}} & \bar{L}_{\delta} & \bar{L}_{\dot{\delta}}
\end{array}\right] \\
\bar{L}_{\delta}=F(k) L_{\delta}^{*}-\bar{L}_{\ddot{\delta}} \omega_{a}^{2} \\
\bar{L}_{\dot{\delta}}=L_{\dot{\delta}}+F(k) L_{\dot{\delta}}^{*}+\frac{G(k)}{\omega} L_{\delta}^{*}-\bar{L}_{\ddot{\delta}} 2 \zeta_{a} \omega_{a}
\end{gathered}
$$

2. We formulate the frequency-independent ASE state-space model with output independent of control $(D=0)$ as follows.

Let $x=\left[\begin{array}{lllllllllllll}q & \dot{q} & y_{q} & \dot{y}_{q} & z_{q} & \dot{z}_{q} & \delta & \dot{\delta} & \ddot{\delta} & y_{\delta} & \dot{y}_{\delta} & z_{\delta} & \dot{z}_{\delta}\end{array}\right]^{\top}$. Then, the state-space model is given by

$$
\begin{gathered}
A=\left[\begin{array}{cc|c}
A_{11} & A_{12} \\
\hline 0 & A_{22}
\end{array}\right] \\
B=\left[\begin{array}{lllllllllllll}
0 & 0 & 0 & 0 & 0 & 0 & 0 & 0 & d k_{i} & 0 & 0 & 0 & 0
\end{array}\right]^{\top} \\
E=\left[\begin{array}{ccccccccccccc}
0 & \bar{M}^{-1} F_{\theta_{v}} & 0 & 0 & 0 & 0 & 0 & 0 & 0 & 0 & 0 & 0 & 0 \\
0 & \bar{M}^{-1} F_{\dot{\theta}_{v}} & 0 & 0 & 0 & 0 & 0 & 0 & 0 & 0 & 0 & 0 & 0
\end{array}\right]^{\top}
\end{gathered}
$$

where

$$
A_{11}=\left[\begin{array}{cccccc}
0 & I & 0 & 0 & 0 & 0 \\
-\bar{M}^{-1} \bar{K} & -\bar{M}^{-1} \bar{C} & -\bar{M}^{-1} K_{a}^{*} & 0 & -\bar{M}^{-1} C_{a}^{*}\left(\frac{2 V_{\infty}}{\bar{c}}\right) & 0 \\
0 & 0 & 0 & I & 0 & 0 \\
0.5 a_{2}\left(\frac{2 V_{\infty}}{\bar{c}}\right)^{2} & a_{4}\left(\frac{2 V_{\infty}}{\bar{c}}\right) & -a_{2}\left(\frac{2 V_{\infty}}{\bar{c}}\right)^{2} & -a_{3}\left(\frac{2 V_{\infty}}{\bar{c}}\right) & 0 & 0 \\
0 & 0 & 0 & 0 & 0 & I \\
a_{6}\left(\frac{2 V_{\infty}}{\bar{c}}\right)^{2} & a_{5}\left(\frac{2 V_{\infty}}{\bar{c}}\right) & 0 & 0 & -a_{2}\left(\frac{2 V_{\infty}}{\bar{c}}\right)^{2} & -a_{3}\left(\frac{2 V_{\infty}}{\bar{c}}\right)
\end{array}\right]
$$




$$
\begin{aligned}
& A_{12}=\left[\begin{array}{ccccccc}
0 & 0 & 0 & 0 & 0 & 0 & 0 \\
\bar{M}^{-1} \bar{F}_{\delta} & \bar{M}^{-1} \bar{F}_{\dot{\delta}} & \bar{M}^{-1} F_{\ddot{\delta}} & \bar{M}^{-1} F_{\delta}^{*} & 0 & \bar{M}^{-1}\left(\frac{2 V_{\infty}}{\bar{c}}\right) F_{\tilde{\delta}}^{*} & 0 \\
0 & 0 & 0 & 0 & 0 & 0 & 0 \\
0 & 0 & 0 & 0 & 0 & 0 & 0 \\
0 & 0 & 0 & 0 & 0 & 0 & 0 \\
0 & 0 & 0 & 0 & 0 & 0 & 0
\end{array}\right] \\
& A_{22}=\left[\begin{array}{ccccccc}
0 & I & 0 & 0 & 0 & 0 & 0 \\
0 & 0 & I & 0 & 0 & 0 & 0 \\
-d k_{i} & -\left(\omega_{a}^{2}+d k_{p}\right) & -\left(2 \zeta_{a} \omega_{a}+d k_{d}\right) & 0 & 0 & 0 & 0 \\
0 & 0 & 0 & 0 & I & 0 & 0 \\
0.5 a_{2}\left(\frac{2 V_{\infty}}{\bar{c}}\right)^{2} & a_{4}\left(\frac{2 V_{\infty}}{\bar{c}}\right) & 0 & -a_{2}\left(\frac{2 V_{\infty}}{\bar{c}}\right)^{2} & -a_{3}\left(\frac{2 V_{\infty}}{\bar{c}}\right) & 0 & 0 \\
0 & 0 & 0 & 0 & 0 & 0 & I \\
a_{6}\left(\frac{2 V_{\infty}}{\bar{c}}\right)^{2} & a_{5}\left(\frac{2 V_{\infty}}{\bar{c}}\right) & 0 & 0 & 0 & -a_{2}\left(\frac{2 V_{\infty}}{\bar{c}}\right)^{2} & -a_{3}\left(\frac{2 V_{\infty}}{\bar{c}}\right)
\end{array}\right] \\
& \bar{F}_{\delta}=F_{\delta}+0.5 F_{\delta}^{*}+a_{4}\left(\frac{2 V_{\infty}}{\bar{c}}\right) F_{\dot{\delta}}^{*} \\
& \bar{F}_{\dot{\delta}}=F_{\dot{\delta}}+0.5 F_{\dot{\delta}}^{*}
\end{aligned}
$$

and $(\bar{M}, \bar{C}, \bar{K})$ are given in Eqs. (78) - (80).

The output vector is described by Eqs. (198) - (200) with $(A, B, E)$ defined by Eqs. (227) - (229) and $I_{\ddot{q}}$ replaced by $I_{\ddot{q}}=\left[\begin{array}{lllllllllllll}0 & I & 0 & 0 & 0 & 0 & 0 & 0 & 0 & 0 & 0 & 0 & 0\end{array}\right]$.

The unsteady lift expression is given by Eqs. (201) - (204) with $\left(L_{x}, \bar{L}_{q}, \bar{L}_{\dot{q}}, \bar{L}_{\ddot{q}}, \bar{L}_{\delta}, \bar{L}_{\dot{\delta}}\right)$ replaced by

$$
\begin{aligned}
& L_{x}=\left[\begin{array}{ccccccccccccc}
\bar{L}_{q} & \bar{L}_{\dot{q}} & L_{q}^{*} & 0 & \left(\frac{2 V_{\infty}}{\bar{c}}\right) L_{\dot{q}}^{*} & 0 & \bar{L}_{\delta} & \bar{L}_{\dot{\delta}} & L_{\ddot{\delta}} & L_{\delta}^{*} & 0 & \left(\frac{2 V_{\infty}}{\bar{c}}\right) L_{\dot{\delta}}^{*} & 0
\end{array}\right] \\
& \bar{L}_{q}=0.5 L_{q}^{*}+a_{4}\left(\frac{2 V_{\infty}}{\bar{c}}\right) L_{\dot{q}}^{*} \\
& \bar{L}_{\dot{q}}=L_{\dot{q}}+0.5 L_{\dot{q}}^{*}+\Delta L_{\dot{q}} \\
& \bar{L}_{\ddot{q}}=L_{\ddot{q}}+\Delta L_{\ddot{q}} \\
& \bar{L}_{\delta}=0.5 L_{\delta}^{*}+a_{4}\left(\frac{2 V_{\infty}}{\bar{c}}\right) L_{\dot{\delta}}^{*} \\
& \bar{L}_{\dot{\delta}}=L_{\dot{\delta}}+0.5 L_{\dot{\delta}}^{*}
\end{aligned}
$$

